\title{
Not so Demanding: Demand Structure and Firm Behavior
}

\author{
By Monika Mrázová and J. Peter Neary*
}

We show that any well-behaved demand function can be represented by its "demand manifold," a smooth curve that relates the elasticity and convexity of demand. This manifold is a sufficient statistic for many comparative statics questions; leads naturally to characterizations of new families of demand functions that nest most of those used in applied economics; and connects assumptions about demand structure with firm behavior and economic performance. In particular, the demand manifold leads to new insights about industry adjustment with heterogeneous firms, and can be empirically estimated to provide a quantitative framework for measuring the effects of globalization.

JEL: D11, F12, L11

Keywords: Heterogeneous Firms; Pass-Through; Quantifying Effects of Globalization; Super- and Sub-Convexity; Supermodularity.

Assumptions about the structure of preferences and demand matter enormously for comparative statics in trade, industrial organization, and many other applied fields. Examples from international trade include competition effects (such as whether globalization reduces firms' markups), which depend on whether the elasticity of demand falls with sales $;^{1}$ and selection effects (such as whether more productive firms select into FDI rather than exports), which depend on whether the elasticity and convexity of demand sum to more than three. ${ }^{2}$ Examples from industrial organization include pass-through (do firms pass on cost increases by more than dollar-for-dollar?), which depends on whether the demand function is $\log$-convex $;^{3}$ and the welfare effects of third-degree price discrimination, which

* Mrázová: University of Geneva and CEPR; Geneva School of Economics and Management (GSEM), University of Geneva, Bd. du Pont d'Arve 40, 1211 Geneva 4, Switzerland; monika.mrazova@unige.ch. Neary: University of Oxford, CEPR and CESifo; Department of Economics, University of Oxford, Manor Road, Oxford OX1 3UQ, UK; peter.neary@economics.ox.ac.uk. Earlier versions were circulated under the title "Not So Demanding: Preference Structure, Firm Behavior, and Welfare." We are grateful to Kevin Roberts for suggestions that substantially improved the paper, and also to three anonymous referees, to Costas Arkolakis, Jim Anderson, Pol Antràs, Andy Bernard, Arnaud Costinot, Jean-Baptiste Coulaud, Simon Cowan, Dave Donaldson, Ernst Hairer, Yang-Hui He, Ron Jones, Paul Klemperer, Arthur Lewbel, Jérémy Lucchetti, Lars Mathiesen, Mark Melitz, Mathieu Parenti, Fred Schroyen, Alain Trannoy, Bart Vandereycken, Jonathan Vogel and Glen Weyl, and to participants at various seminars and conferences, for helpful comments. Monika Mrázová thanks the Fondation de Famille Sandoz for funding under the "Sandoz Family Foundation - Monique de Meuron" Programme for Academic Promotion. Peter Neary thanks the European Research Council for funding under the European Union's Seventh Framework Programme (FP7/2007-2013), ERC grant agreement no. 295669. The authors declare that they have no relevant or material financial interests that relate to the research described in this paper.

${ }^{1}$ See Krugman (1979) and Zhelobodko et al. (2012).

${ }^{2}$ See Helpman, Melitz and Yeaple (2004) and Mrázová and Neary (2018).

${ }^{3}$ See Bulow and Pfleiderer (1983) and Weyl and Fabinger (2013). 
depend on how demand convexity varies with price. ${ }^{4}$ In all these cases, the answer to an important real-world question hinges on a feature of demand that seems at best arbitrary and in some cases esoteric. All bar specialists may have difficulty remembering these results, far less explicating them and relating them to each other.

There is an apparent paradox here. These applied questions are all supply-side puzzles: they concern the behavior of firms or the performance of industries. Why then should the answers to them hinge on the shape of demand functions, and in many cases on their second or even third derivatives? However, as is well known, the paradox is only apparent. In perfectly competitive models, shifts in supply curves lead to movements along the demand curve, and so their effects hinge on the slope or elasticity of demand. When firms are monopolists or monopolistic competitors, as in this paper, they do not have a supply function as such; instead, exogenous supply-side shocks or differences between firms lead to more subtle differences in behavior, whose implications depend on the curvature as well as the slope of the demand function.

Different authors and even different sub-fields have adopted a variety of approaches to these issues. Weyl and Fabinger (2013) show that many results can be understood by taking the degree of pass-through of costs to prices as a unifying principle. Macroeconomists frequently work with the "superelasticity" of demand, due to Kimball (1995), to model more realistic patterns of price adjustment than allowed by CES preferences. In our previous work (Mrázová and Neary (2018)), we showed that, since monopoly firms adjust along their marginal revenue curve rather than the demand curve, the elasticity of marginal revenue itself pins down some results. Each of these approaches focuses on a single demand measure that is a sufficient statistic for particular results. This paper goes much further than these, by developing a general framework that provides a new perspective on how assumptions about the functional form of demand determine conclusions about comparative statics.

The key idea we explore is the value of taking a "firm's-eye view" of demand functions. To understand a monopoly firm's responses to infinitesimal shocks it is enough to focus on the local properties of the demand function it faces, since these determine its choice of output: the slope of demand determines the firm's level of marginal revenue, which it wishes to equate to marginal cost, while the curvature of demand determines the slope of marginal revenue, which must be negative if the second-order condition for profit maximization is to be met. Measuring slope and curvature in unit-free ways leads us to focus on the elasticity and convexity of demand, following Seade (1980), and we show that for any well-behaved demand function these two parameters are related to each other. We call the implied relationship the "demand manifold", and show that it is a sufficient statistic linking the functional form of demand to many comparative statics properties. It thus allows us to develop new comparative statics results and illustrate existing

\footnotetext{
${ }^{4}$ See Schmalensee (1981) and Aguirre, Cowan and Vickers (2010).
} 
ones in a simple and compact way; and it leads naturally to characterizations of new families of demand functions that provide a parsimonious way of nesting existing ones, including most of those used in applied economics. ${ }^{5}$

A "firm's-eye view" is partial-equilibrium by construction, of course. Nevertheless, it can provide the basis for understanding general-equilibrium behavior. To demonstrate this, we show how our approach allows us to characterize the responses of outputs, prices and product variety in the canonical model of international trade under monopolistic competition due to Krugman (1979). We show how the quantitative magnitude of the model's properties can be related to the assumed demand function through the lens of the implied demand manifold. Furthermore, we use our approach to derive new results for the case of heterogeneous firms, as in Melitz (2003), extended to general demands, as in Zhelobodko et al. (2012), Bertoletti and Epifani (2014) and Dhingra and Morrow (2016). Following Dixit and Stiglitz (1977), we concentrate on the case of additively separable preferences, but our "firms'-eye perspective" can also be applied to other forms of monopolistic competition, as in Dixit and Stiglitz (1993), Feenstra (2014), Bertoletti and Etro (2016), Bertoletti and Etro (2017) and Parenti, Ushchev and Thisse (2017).

While the demand manifold is a theoretical construct, it also has potential empirical uses. In particular, it allows us to infer the parameters needed for comparative statics and counterfactual exercises, without estimating a demand function. We show that, given estimates of pass-through and markups, it is possible to back out the implied form of the demand manifold. With additional assumptions we can go further. Assuming that preferences are additively separable makes it possible to infer the implied income elasticities, while assuming parametric forms of demand opens the door towards quantifying the gains from trade.

The plan of the paper follows this route map. Section I introduces our new perspective on demand, and shows how the elasticity and convexity of demand condition comparative statics results. Section II shows how the demand manifold can be located in the space of elasticity and convexity, and explores how a wide range of demand functions, both old and new, can be represented by their manifold in a parsimonious way. Section III illustrates the usefulness of our approach by applying it to a canonical general-equilibrium model of international trade under monopolistic competition, and characterizing the implications of assumptions about functional form for the quantitative effects of exogenous shocks. Section IV turns to show how the demand manifold can be empirically estimated, and how it can be used for counterfactual analysis. Section V concludes, Appendix A gives some technical background and discusses some extensions, while online Appendix B gives proofs of all propositions, discusses some further extensions, and provides a glossary of terms used.

\footnotetext{
${ }^{5}$ Demand functions used in recent work that fit into our framework include the linear (Melitz and Ottaviano (2008)), LES (Simonovska (2015)), CARA (Behrens and Murata (2007)), translog (Feenstra (2003)), QMOR (Feenstra (2014)), and Bulow-Pfleiderer (Atkin and Donaldson (2012)). See Section II.D and Appendices B8 and B9.
} 


\section{Demand Functions and Comparative Statics}

\section{A. A Firm's-Eye View of Demand}

A perfectly competitive firm takes the price it faces as given. Our starting point is the fact that a monopolistic or monopolistically competitive firm takes the demand function it faces as given. Observing economists will often wish to solve for the full general equilibrium of the economy, or to consider the implications of alternative assumptions about the structure of preferences (such as discrete choice, representative agent, homotheticity, separability, etc.); we will consider many such examples in later sections. By contrast, the firm takes all these as given and is concerned only with maximizing profits subject to the partial-equilibrium demand function it perceives. In this section, we consider the implications of this "firm's-eye view" of demand. For the most part we write the demand function in inverse form, $p=p(x)$, with the only restrictions that consumers' willingness to pay is continuous, three-times differentiable, and strictly decreasing in sales: $p^{\prime}(x)<0 .^{6}$ It is sometimes convenient to switch to the corresponding direct demand function, $x=x(p)$, with $x^{\prime}(p)<0$, the inverse of $p(x)$.

As explained in the introduction, we express all our results in terms of the slope and curvature of demand, measured by two unit-free parameters, the elasticity $\varepsilon$ and convexity $\rho$ of the demand function:

$$
\varepsilon(x) \equiv-\frac{p(x)}{x p^{\prime}(x)}>0 \quad \text { and } \quad \rho(x) \equiv-\frac{x p^{\prime \prime}(x)}{p^{\prime}(x)}
$$

These are not unique measures of slope and curvature, and our results could alternatively be presented in terms of other parameters, such as the convexity of the direct demand function, or the Kimball (1995) superelasticity of demand. Appendix A1 gives more details of these alternatives, and explains our preference for focusing on $\varepsilon$ and $\rho$.

Because we want to highlight the implications of alternative assumptions about demand, we assume throughout that marginal cost is constant. ${ }^{7}$ Maximizing profits therefore requires that marginal revenue should equal marginal cost and should be decreasing with output. This imposes restrictions on the values of $\varepsilon$ and $\rho$ that must hold at a profit-maximizing equilibrium. From the first-order condition, a non-negative price-cost margin implies that the elasticity must be greater than one:

$$
p+x p^{\prime}=c \geq 0 \Rightarrow \varepsilon \geq 1
$$

From the second-order condition, marginal revenue $p+x p^{\prime}$ decreasing with output

\footnotetext{
${ }^{6}$ We use "sales" throughout to denote consumption $x$, which in equilibrium equals the firm's output.

${ }^{7}$ Zhelobodko et al. (2012) show that variable marginal costs make little difference to the properties of models with homogeneous firms. In models of heterogeneous firms it is standard to assume that marginal costs are constant.
} 
implies that our measure of convexity must be strictly less then two:

$$
2 p^{\prime}+x p^{\prime \prime}<0 \Rightarrow \rho<2
$$

These restrictions imply an admissible region in $\{\varepsilon, \rho\}$ space, as shown by the shaded region in Figure 1(a). ${ }^{8}$ Consumers may be willing to consume outside the admissible region, but such points cannot represent the profit-maximizing equilibrium of a monopoly or monopolistically competitive firm.

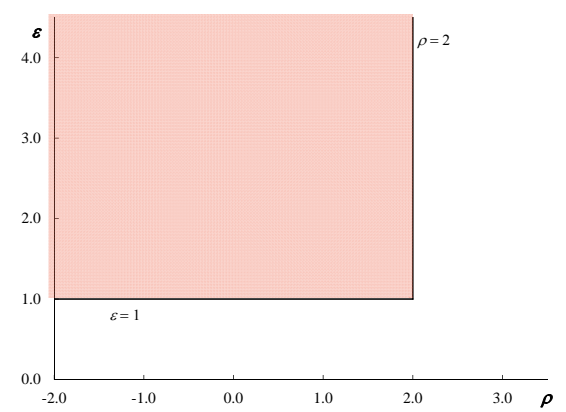

(a) The Admissible Region

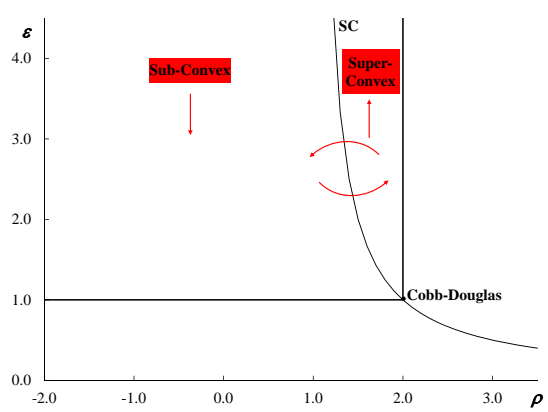

(b) The Super- and Sub-Convex Regions

Figure 1. The Space of Elasticity and Convexity

\section{B. The CES Benchmark}

In general, both $\varepsilon$ and $\rho$ vary with sales. The only exception is the case of CES preferences or iso-elastic demands: ${ }^{9}$

$$
p(x)=\beta x^{-1 / \sigma} \Rightarrow \varepsilon=\sigma, \quad \rho=\rho^{C E S} \equiv \frac{\sigma+1}{\sigma}>1
$$

Clearly this case is very special: both elasticity and convexity are determined by a single parameter, $\sigma$. Eliminating this parameter gives a relationship between $\varepsilon$ and $\rho$ that must hold for all members of the CES family: $\varepsilon=1 /(\rho-1)$, or $\rho=(\varepsilon+1) / \varepsilon$. This is illustrated by the curve labeled "SC" in Figure 1(b). Every

${ }^{8}$ The admissible region is $\{(\varepsilon, \rho): 1 \leq \varepsilon \leq \infty$ and $-\infty \leq \rho<2\}$. In the figures that follow, we illustrate the subset of the admissible region where $\varepsilon<4.5$ and $\rho \geq-2.0$, since this is where most interesting issues arise and it is also consistent with the available empirical evidence. (Broda and Weinstein (2006), Soderbery (2015) and Benkovskis and Wörz (2014) estimate median elasticities of demand for imports of 3.7 or lower.) Note that the admissible region is larger in oligopolistic markets, since both boundary conditions are less stringent than (2) and (3). See Appendix A2 for details.

${ }^{9}$ It is convenient to follow the widespread practice of applying the "CES" label to the demand function in (4), though this only follows from CES preferences in the case of monopolistic competition, when firms assume they cannot affect the aggregate price index. The fact that CES demands are sufficient for constant elasticity is obvious. The fact that they are necessary follows from setting $-p(x) / x p^{\prime}(x)$ equal to a constant $\sigma$ and integrating. 
point on this curve corresponds to a different CES demand function: firms always operate at that point irrespective of the values of exogenous variables. In this respect too the CES is very special, as we will see. The Cobb-Douglas special case corresponds to the point $\{\varepsilon, \rho\}=\{1,2\}$, and so has the dubious distinction of being just on the boundary of both the first- and second-order conditions.

The CES case is important in itself but also because it is an important boundary for comparative statics results. Following Mrázová and Neary (2018), we say that a demand function is "superconvex" at an arbitrary point if it is more convex at that point than a CES demand function with the same elasticity. Hence the eponymous SC curve in Figure 1(b) divides the admissible region in two: points to the right are strictly superconvex, points to the left are strictly subconvex, while all CES demand functions are both weakly superconvex and weakly subconvex. As we show in Appendix B1, superconvexity also determines the relationship between demand elasticity and sales: the elasticity of demand increases in sales (or, equivalently, decreases in price), $\varepsilon_{x} \geq 0$, if and only if the demand function $p(x)$ is superconvex. So, $\varepsilon$ is independent of sales only along the SC locus, it increases with sales in the superconvex region to the right, and decreases with sales in the subconvex region to the left. ${ }^{10}$ These properties imply something like the comparative-statics analogue of a phase diagram: the arrows in Figure 1(b) indicate the direction of movement as sales rise. ${ }^{11}$

\section{Illustrating Comparative Statics Results}

We can use our diagram to illustrate some of the comparative statics results discussed in the introduction. The results themselves are not new, but illustrating them in a common framework provides new insights and sets the scene for our discussion of the implications of particular demand functions in Section II.

\section{Competition Effects and Relative Pass-Through: Superconvexity}

Superconvexity itself determines both competition effects and relative passthrough: the effects of globalization and of cost changes respectively on firms' proportional profit margins. From the first-order condition, the relative markup or proportional profit margin $m \equiv(p-c) / c$ equals $-x p^{\prime} /\left(p+x p^{\prime}\right)$, which is inversely related to the elasticity of demand: $m=1 /(\varepsilon-1)$. Hence, if globalization reduces incumbent firms' sales in their home markets, it is associated with a higher

\footnotetext{
${ }^{10}$ Many authors, including Marshall (1920), Dixit and Stiglitz (1977), and Krugman (1979), have argued that subconvexity is intuitively more plausible. (It is sometimes called "Marshall's Second Law of Demand." See Appendix B19 for further discussion.) Moreover, subconvexity is consistent with much of the available empirical evidence on proportional pass-through, which suggests that it is less than 100\%. See for example Gopinath and Itskhoki (2010), De Loecker et al. (2016), and our discussion in Section IV.A below. However, superconvexity cannot be ruled out either theoretically or empirically: as Zhelobodko et al. (2012) point out, some empirical studies find that entry or economic integration leads to higher markups. See, for example, Ward et al. (2002) and Badinger (2007).

${ }^{11}$ For most widely-used demand functions, the implied points in this space are always on one or other side of the SC curve. See Section II for further discussion and Appendix B10 for a counter-example.
} 


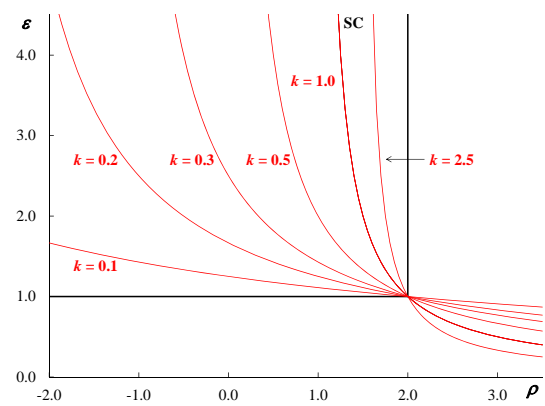

(a) Constant Proportional Pass-Through

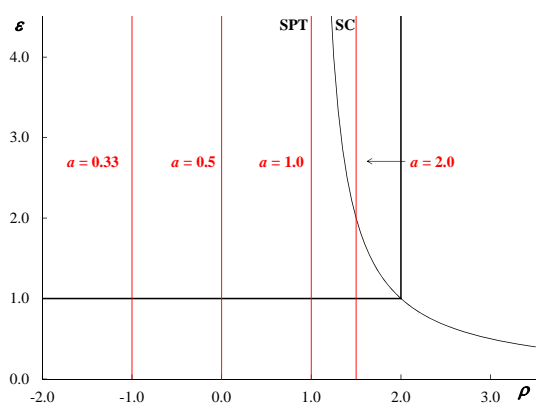

(b) Constant Absolute Pass-Through

Figure 2. Loci of Constant Pass-Through

elasticity and so a lower markup if and only if demand is subconvex. Similarly, an increase in marginal cost $c$, which other things equal must lower sales, is associated with a higher elasticity and so a lower markup, implying less than $100 \%$ pass-through, if and only if demands are subconvex:

(5) $\frac{d \log p}{d \log c}=\frac{\varepsilon-1}{\varepsilon} \frac{1}{2-\rho}>0 \Rightarrow \frac{d \log p}{d \log c}-1=\frac{d \log p}{d \log c}-1=-\frac{\varepsilon+1-\varepsilon \rho}{\varepsilon(2-\rho)} \gtreqless 0$

More generally, loci corresponding to $100 k \%$ pass-through, i.e., $d \log p / d \log c=k$, are defined by: ${ }^{12}$

$$
\rho=2-\frac{1}{k} \frac{\varepsilon-1}{\varepsilon}
$$

Figure 2(a) illustrates some of these loci for different values of $k$.

\section{Absolute Pass-Through: Log-Convexity}

The criterion for absolute or dollar-for-dollar pass-through from cost to price has been known since Bulow and Pfleiderer (1983). Differentiating the first-order condition $p+x p^{\prime}=c$, we see that an increase in cost must raise price provided only that the second-order condition holds, which implies an expression for the effect of an increase in marginal cost on the absolute profit margin that is different from the proportional pass-through expression in (5):

$$
\frac{d p}{d c}=\frac{1}{2-\rho}>0 \quad \Rightarrow \quad \frac{d p}{d c}-1=\frac{\rho-1}{2-\rho} \gtreqless 0
$$

\footnotetext{
${ }^{12}$ This is a family of rectangular hyperbolas, all asymptotic to $\{\varepsilon, \rho\}=\{\infty,(2 k-1) / k\}$ and $\{0, \infty\}$, and all passing through the Cobb-Douglas point $\{\varepsilon, \rho\}=\{1,2\}$. We discuss this family further in Section II.E below.
} 
Hence we have what we call "Super-Pass-Through", whereby the equilibrium price rises by more than the increase in marginal cost, if and only if $\rho$ is greater than one. More generally, loci corresponding to a pass-through coefficient of $a$ are defined by convexity values of $\rho=2-1 / a$. Figure 2(b) illustrates some of these loci for different values of $a$. The one corresponding to $a=1$, labeled "SPT", divides the admissible region into sub-regions of sub- and super-pass-through. It corresponds to a log-linear direct demand function, which is less convex than the CES. ${ }^{13}$ Hence superconvexity implies super-pass-through, but not the converse: in the region between the SPT and SC loci, pass-through is more than dollarfor-dollar but less than 100\%. More generally, comparing Figures 2(a) and 2(b) shows that at any point the degree of absolute pass-through is greater than that of relative pass-through, and by more so the lower the elasticity; the implied relationship is: $a / k=\varepsilon /(\varepsilon-1)$.

\section{Selection Effects: Supermodularity}

A third criterion for comparative statics responses that we can locate in our diagram arises in models with heterogeneous firms, where firms choose between two alternative ways of serving a market, such as the choice between exports and foreign direct investment (FDI) as in Helpman, Melitz and Yeaple (2004). ${ }^{14}$ Mrázová and Neary (2018) show that more efficient firms are sure to select into FDI only if their ex post profit function is supermodular in their own marginal cost $c$ and the iceberg transport cost they face $t$. Supermodularity holds if and only if the elasticity of marginal revenue with respect to sales is less than one, which in turn implies that the elasticity and convexity of demand sum to more than three. ${ }^{15}$ When this condition holds, a $10 \%$ reduction in the marginal cost of serving a market raises sales by more than $10 \%$, so more productive firms have a greater incentive to engage in FDI than in exports. This criterion defines a third locus in $\{\varepsilon, \rho\}$ space, as shown by the straight line labeled "SM" in Figure 3. Once again it divides the admissible region into two sub-regions, one where either the elasticity or convexity or both are high, so supermodularity prevails, and the other where the profit function is submodular. The locus lies everywhere below the superconvex locus, and is tangential to it at the Cobb-Douglas point. Hence, supermodularity always holds with CES demands. However, when demands are

\footnotetext{
${ }^{13}$ Setting $\rho=1$ implies a second-order ordinary differential equation $x p^{\prime \prime}(x)+p^{\prime}(x)=0$. Integrating this yields $p(x)=c_{1}+c_{2} \log x$, where $c_{1}$ and $c_{2}$ are constants of integration, which is equivalent to a $\log$-linear direct demand function, $\log x(p)=\gamma+\delta p$.

${ }^{14}$ Mrázová and Neary (2018) show that the same criterion determines selection effects in a number of other cases, including the choice between producing in the high-wage "North" or the low-wage "South" as in Antràs and Helpman (2004), and the choice of technique as in Bustos (2011). Related applications can be found in Spearot (2012) and Spearot (2013).

${ }^{15}$ Let $\pi(c, t) \equiv \max _{x}[p(x)-t c] x$ denote the maximum operating profits which a firm with marginal production cost $c$ can earn facing an iceberg transport cost of accessing the market equal to $t$. When $\pi$ is twice differentiable, supermodularity implies that $\pi_{c t}$ is positive. By the envelope theorem, $\pi_{c}=-t x$. Hence, $\pi_{c t}=-x-t(d x / d t)=-x-t c /\left(2 p^{\prime}+x p^{\prime \prime}\right)=-x+x(\varepsilon-1) /(2-\rho)$. Writing revenue as $R(x)=x p(x)$, so marginal revenue is $R^{\prime}=p+x p^{\prime}$, the elasticity of marginal revenue (in absolute value) is seen to be: $-x R^{\prime \prime} / R^{\prime}=(2-\rho) /(\varepsilon-1)$. The results in the text follow by inspection.
} 
subconvex and firms are large (operating at a point on their demand curve with relatively low elasticity), submodularity prevails, and so the standard selection effects may be reversed.

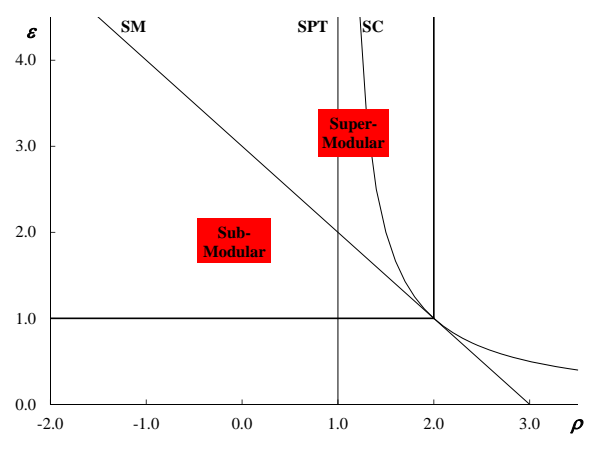

Figure 3. The Super- And Sub-Modular Regions

\section{Summary}

Figure 4 summarizes the results illustrated in this section. The three loci, corresponding to constant elasticity (SC), unit convexity (SPT), and unit elasticity of marginal revenue (SM), place bounds on the combinations of elasticity and convexity consistent with particular comparative statics outcomes. Of eight logically possible sub-regions within the admissible region, three can be ruled out because superconvexity implies both super-pass-through and supermodularity. From the figure it is clear that knowing the values of the elasticity and convexity of demand that a firm faces is sufficient to predict its responses to a wide range of exogenous shocks, including some of the classic questions posed in the introduction.

\section{The Demand Manifold}

So far, we have shown how a wide range of comparative statics responses can be signed just by knowing the values of $\varepsilon$ and $\rho$ that a firm faces. Next we want to see how different assumptions about the form of demand determine these responses. To do this, Section II.A introduces our key innovation, the "demand manifold" corresponding to a particular demand function. We show that, in all cases other than the CES, the manifold is represented by a smooth curve in $(\varepsilon, \rho)$ space. Section II.B derives the conditions which guarantee that the manifold is invariant with respect to shifts in the demand function. Sections II.C and II.D show how many widely-used demand functions can be parsimoniously represented by their demand manifolds, which provides a simple unifying principle for a very wide range of applications. Section II.E then shows how the demand manifold 


\begin{tabular}{cccc}
\hline Region & $\varepsilon_{x}>0$ & $\frac{d p}{d c}>0$ & $\varepsilon+\rho>3$ \\
\hline 1 & & & $\checkmark$ \\
2 & & $\checkmark$ & \\
3 & & $\checkmark$ & $\checkmark$ \\
4 & & $\checkmark$ & $\checkmark$ \\
5 & $\checkmark$ & & \\
\hline
\end{tabular}

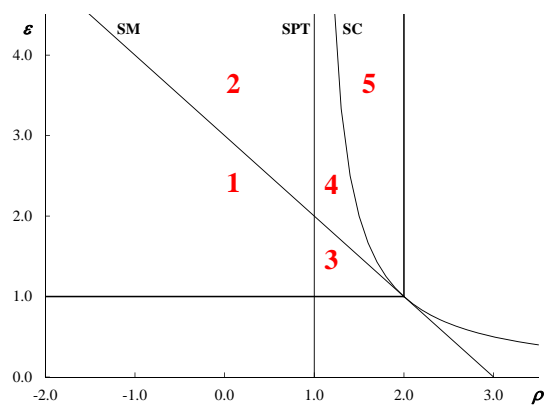

Figure 4. Regions of Comparative Statics

can be used to infer the comparative statics implications of a particular demand function, while Section II.F notes some demand functions whose manifolds are not invariant with respect to any of their parameters.

\section{A. Demand Functions and Demand Manifolds}

Formally, we seek to characterize the set of values of the elasticity $\varepsilon$ and convexity $\rho$ that are consistent with a particular demand function $p_{0}: x \mapsto p_{0}(x)$ :

DEFINITION 1 (Definition of the Demand Manifold):

$$
\Omega_{p_{0}} \equiv\left\{(\varepsilon, \rho): \varepsilon=-\frac{p_{0}(x)}{x p_{0}^{\prime}(x)}, \rho=-\frac{x p_{0}^{\prime \prime}(x)}{p_{0}^{\prime}(x)}, \forall x \in X_{p_{0}}\right\}
$$

where the domain of $p_{0}$ is such that both output $x$ and price $p$ are non-negative: $X_{p_{0}} \equiv\left\{x: x \geq 0\right.$ and $\left.p_{0}(x) \geq 0\right\} \subset \mathbb{R}_{\geq 0}$. We have already seen that the set $\Omega_{p_{0}}$, and hence the comparative statics responses implied by particular demand functions, are pinned down in one special case: facing a particular CES demand function, the firm is always at a single point in $(\varepsilon, \rho)$ space. Can anything be said more generally? The answer is "yes", as the following result shows.

PROPOSITION 1 (Existence of the Demand Manifold): For every continuous, three-times differentiable, strictly-decreasing demand function, $p_{0}(x)$, other than the CES, the set $\Omega_{p_{0}}$ corresponds to a smooth curve in $(\varepsilon, \rho)$ space.

The proof is in Appendix B2. It proceeds by showing that, at any point on every demand function other than the CES, at least one of the functions $\varepsilon=\varepsilon(x)$ and $\rho=\rho(x)$ can be inverted to solve for $x$, and the resulting expression, denoted $x^{\varepsilon}(\varepsilon)$ and $x^{\rho}(\rho)$ respectively, substituted into the other function to give a relationship between $\varepsilon$ and $\rho$ :

$$
\varepsilon=\bar{\varepsilon}(\rho) \equiv \varepsilon\left[x^{\rho}(\rho)\right] \quad \text { or } \quad \rho=\bar{\rho}(\varepsilon) \equiv \rho\left[x^{\varepsilon}(\varepsilon)\right]
$$


We write this in two alternative ways, since at any given point only one may be well-defined, and, even when both are well-defined, one or the other may be more convenient depending on the context. The relationship between $\varepsilon$ and $\rho$ defined implicitly by (8) is not in general a function, since it need not be globally singlevalued; but neither is it a correspondence, since it is locally single-valued. This is why we call it the "demand manifold" corresponding to the demand function $p_{0}(x)$. In the CES case, not covered by Proposition 1 , we follow the convention that, corresponding to each value of the elasticity of substitution $\sigma$, the set $\Omega_{p_{0}}$ is represented by a point-manifold lying on the SC locus.

The first advantage of working with the demand manifold rather than the demand function itself is that it is located in $(\varepsilon, \rho)$ space, and so it immediately reveals the implications of assumptions made about demand for comparative statics. A second advantage, departing from the "firm's-eye view" that we have adopted so far, is that the manifold is often independent of exogenous parameters even though the demand function itself is not. Expressing this in the language of Chamberlin (1933), exogenous shocks typically shift the perceived demand curve, but they need not shift the corresponding demand manifold. We call this property "manifold invariance". When it holds, exogenous shocks lead only to movements along the manifold, not to shifts in it. As a result, it is particularly easy to make comparative statics predictions.

\section{B. Manifold Invariance}

We wish to characterize the conditions under which manifold invariance holds. Clearly, the manifold cannot in most cases be invariant to changes in all parameters: even in the CES case, the point-manifold is not independent of the value of $\sigma .{ }^{16}$ However, the CES point-manifold is invariant to changes in any parameter $\phi$ that affects the level term only; for ease of comparison with later functions, we write this in terms of both the inverse and direct CES demand functions: ${ }^{17}$

$$
\text { (a) } p(x, \phi)=\beta(\phi) x^{-1 / \sigma} \Leftrightarrow \quad \text { (b) } x(p, \phi)=\delta(\phi) p^{-\sigma}
$$

It is particularly convenient that the CES point-manifold is invariant with respect to variables (such as income or the prices of other goods) that are endogenous in general equilibrium and affect only the level term, whereas the parameter $\sigma$ with respect to which it is not invariant is a structural preference parameter. In the same way, as we show formally in Corollary 2 below, the manifold corresponding to any demand function turns out to be invariant with respect to its level parameter.

It is very desirable to have both necessary and sufficient conditions for a demand manifold to be invariant with respect to a particular parameter, and these

\footnotetext{
${ }^{16}$ Demand functions whose manifolds are invariant with respect to all demand parameters are relatively rare, though they include some well-known cases, including linear, Stone-Geary, CARA, and translog demands: see Section II.D below.

${ }^{17}$ These are equivalent, with $\beta(\phi)=\delta(\phi)^{1 / \sigma}$.
} 
are given by Proposition 2. Note that the proposition distinguishes between restrictions derived from inverse and direct demand functions (denoted (a) and (b) respectively). This was not necessary in the definition of the manifold in (8) and the proof of its existence in Proposition 1. However, it is needed here, because in general the responses of the elasticity and convexity of demand to a parameter change depend on whether price or quantity is assumed fixed.

PROPOSITION 2 (Manifold Invariance): Assume that $\rho_{x}$ is non-zero. Then, the demand manifold is invariant with respect to a vector parameter $\phi$ if and only if both $\varepsilon$ and $\rho$ depend on $x$ and $\phi$ through a common sub-function of either (a) $x$ and $\phi$; or (b) $p$ and $\phi$; i.e.:

$$
\begin{aligned}
& \varepsilon(x, \phi)=\tilde{\varepsilon}[F(x, \phi)] \quad \text { and } \quad \rho(x, \phi)=\tilde{\rho}[F(x, \phi)] ; \quad \text { or } \\
& \varepsilon(p, \phi)=\tilde{\varepsilon}[G(p, \phi)] \quad \text { and } \quad \rho(p, \phi)=\tilde{\rho}[G(p, \phi)]
\end{aligned}
$$

The proof is in Appendix B3.

To understand this result, note first that, just as Proposition 1 excluded the CES case where demand elasticity $\varepsilon$ is independent of $x$, so Proposition 2 excludes the case where demand convexity $\rho$ is independent of $x$. The class of demand functions that exhibits this property (which includes CES as a special case) is known as Bulow-Pfleiderer demands, and is considered separately in Section II.D below. Excluding this class, the proposition states that the only other case consistent with manifold invariance is where both $\varepsilon$ and $\rho$ satisfy a separability restriction, such that they depend on $\phi$ and on $x$ or $p$ via a common sub-function, $F(x, \phi)$ or $G(p, \phi)$. A useful corollary is where either $F$ or $G$ themselves is independent of $\phi$, which we can restate as follows:

COROLLARY 1: The demand manifold is invariant with respect to a vector parameter $\phi$ if both elasticity $\varepsilon$ and convexity $\rho$ are independent of $\phi$.

The next two sub-sections illustrate these results. Section II.C extends the CES demand functions from (10) in a non-parametric way and illustrates Corollary 1, while Section II.D extends them parametrically by adding an additional powerlaw term and illustrates the general result in Proposition 2.

\section{Multiplicatively Separable Demand Functions}

Our first result is that manifold invariance holds when the demand function is multiplicatively separable in $\phi$ :

COROLLARY 2: The demand manifold is invariant to shocks in a parameter $\phi$ if either (a) the inverse demand function or (b) the direct demand function is multiplicatively separable in $\phi$ :

$$
\begin{aligned}
& p(x, \phi)=\beta(\phi) \tilde{p}(x) ; \quad \text { or } \\
& x(p, \phi)=\delta(\phi) \tilde{x}(p)
\end{aligned}
$$


The proof is in Appendix B4, and relies on the convenient property that, with separability of this kind, both the elasticity and convexity are themselves invariant with respect to $\phi$, so Corollary 1 immediately applies.

This result has some important implications. First, when utility is additively separable, the inverse demand function for any good equals the marginal utility of that good times the inverse of the marginal utility of income. The latter is a sufficient statistic for all economy-wide variables that affect the demand in an individual market, such as aggregate income or the price index. A similar property holds for the direct demand function if the indirect utility function is additively separable (as in Bertoletti and Etro (2017)), with the qualification that the indirect sub-utility functions depend on prices relative to income. (See Appendix B4 for details.) Summarizing:

COROLLARY 3: If preferences are additively separable, whether directly or indirectly, the demand manifold for any good is invariant to changes in aggregate variables (except for income, in the case of indirect additivity).

Given the pervasiveness of additive separability in theoretical models of monopolistic competition, this is an important result, which implies that in many models the manifold is invariant to economy-wide shocks. We will see a specific application in Section III, where we apply our approach to the Krugman (1979) model of international trade with monopolistic competition.

A second implication of Corollary 2 comes by noting that, setting $\delta(\phi)$ in $(12 \mathrm{~b})$ equal to market size $s$, yields the following:

COROLLARY 4: The demand manifold is invariant to neutral changes in market size: $x(p, s)=s \tilde{x}(p)$.

This corollary is particularly useful since it does not depend on the functional form of the individual demand function $\tilde{x}(p)$. An example that illustrates this is the logistic direct demand function: see Appendix B5 for details.

Finally, a third implication of Corollary 2 is the dual of Corollary 4, and comes from setting $\beta(\phi)$ in (12a) equal to quality $q$ :

COROLLARY 5: The demand manifold is invariant to neutral changes in quality: $p(x, q)=q \tilde{p}(x)$.

This implies that quality affects demand $x$ only through the quality-adjusted price $p / q$. Baldwin and Harrigan (2011) call this assumption "box-size quality": the consumer's willingness to pay for a single box of a good with quality level $q$ is the same as their willingness to pay for $q$ boxes of the same good with unit quality. Though special, it is a very convenient assumption, widely used in international trade, so it is useful that the comparative statics predictions of any such demand function are independent of the level of quality. 


\section{Bipower Demand Functions}

A second class of demand functions that exhibit manifold invariance comes from adding a second power-law term to the CES case (10), giving a "Bipower" or "Double CES" form. The corresponding manifolds can be written in closed form, as Proposition 3 shows:

PROPOSITION 3 (Bipower Demands): The demand manifold is invariant to shocks in a parameter $\phi$ if either (a) the inverse demand function or (b) the direct demand function takes a bipower form:

$$
\begin{array}{lll}
p(x, \phi)=\alpha(\phi) x^{-\eta}+\beta(\phi) x^{-\theta} & \Leftrightarrow & \bar{\rho}(\varepsilon)=\eta+\theta+1-\eta \theta \varepsilon \\
x(p, \phi)=\gamma(\phi) p^{-\nu}+\delta(\phi) p^{-\sigma} & \Leftrightarrow & \bar{\rho}(\varepsilon)=\frac{\nu+\sigma+1}{\varepsilon}-\frac{\nu \sigma}{\varepsilon^{2}}
\end{array}
$$

The proof is in Appendix B6. Clearly, the manifolds in (13a) and (13b) are invariant with respect to the level parameters $\{\alpha, \beta\}$ and $\{\gamma, \delta\}$, so changes in exogenous variables such as income or market size that only affect these parameters do not shift the manifold. (Hence we can suppress $\phi$ from here on.) Putting this differently, we need four parameters to characterize each demand function, but only two to characterize the corresponding manifold, which allows us to place bounds on the comparative statics responses reviewed in Section I.

However, the level parameters in (13a) and (13b) are also qualitatively important, as the following proposition shows:

PROPOSITION 4 (Superconvexity of Bipower Demands): The bipower inverse demand functions in (13a) are superconvex if and only if both $\alpha$ and $\beta$ are positive. Similarly, the bipower direct demand functions in (13b) are superconvex if and only if both $\gamma$ and $\delta$ are positive.

The proof is in Appendix B7. ${ }^{18}$ The two sets of parameters thus play very different roles. The power-law exponents $\{\eta, \theta\}$ and $\{\nu, \sigma\}$ determine the location of the manifold, whereas the level parameters $\{\alpha, \beta\}$ and $\{\gamma, \delta\}$ determine which "branch" of a particular manifold is relevant: the superconvex branch if they are both positive, the subconvex one if either of them is negative. (They cannot both be negative since both price and output are nonnegative.) How this works is best understood by considering some special cases, that, as we will see, include some of the most widely-used demand functions in applied economics.

\footnotetext{
${ }^{18}$ This result has implications for the case where the direct demand function arises from aggregating across two groups with different CES preferences, with elasticities of substitution equal to $\nu$ and $\sigma$, respectively. Now the parameters $\gamma$ and $\delta$ depend inter alia on the weights of the two groups in the population, so both are positive and the market demand function must be superconvex.
} 
Two special cases of the bipower direct class (13b) are of particular interest. ${ }^{19}$ The first, where $\nu=0$, is the family of demand functions due to Pollak (1971). ${ }^{20}$ The direct demand function is now a "translated" CES function of price: $x(p)=$ $\gamma+\delta p^{-\sigma}$; while the demand manifold is a rectangular hyperbola: $\bar{\rho}(\varepsilon)=(\sigma+1) / \varepsilon$. Figure 5(a) illustrates some members of the Pollak family. They include many widely-used demand functions, including the CES $(\gamma=0)$, linear $(\sigma=-1)$, Stone-Geary (or "LES" for "Linear Expenditure System": $\sigma=1$ ), and "CARA" ("constant absolute risk aversion": the limiting case as $\sigma$ approaches zero). ${ }^{21}$ Manifolds with $\sigma$ greater than one have two branches, one each in the sub- and superconvex regions, implying different directions of adjustment as sales increase, as indicated by the arrows. ${ }^{22}$

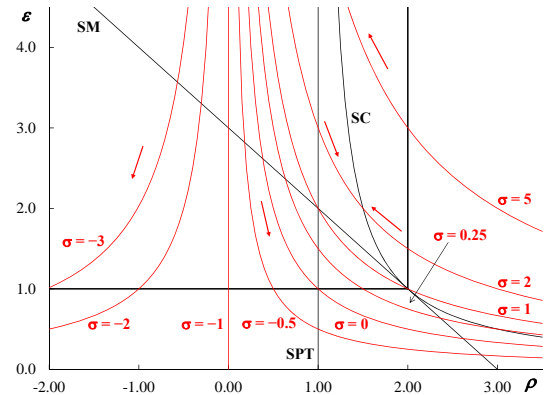

(a) The Pollak Family

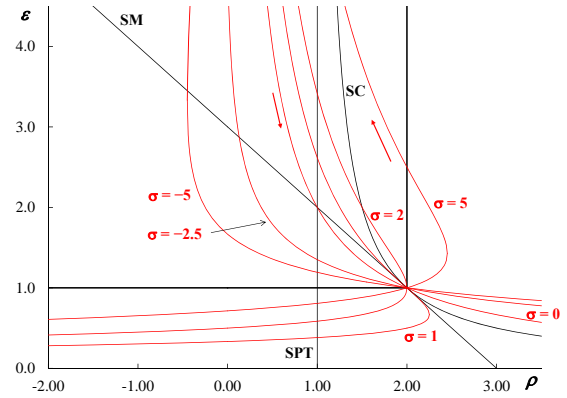

(b) The PIGL Family

Figure 5. Demand Manifolds for Some Bipower Direct Demand Functions

A second important special case of $(13 \mathrm{~b})$ comes from setting $\nu=1$. This gives the "PIGL" ("price-independent generalized linear") system of Muellbauer (1975): $x(p)=\left(\gamma+\delta p^{1-\sigma}\right) / p$, which implies that expenditure $p x(p)$ is a translatedCES function of price. From (13b), the manifold is given by: $\bar{\rho}(\varepsilon)=((\sigma+2) \varepsilon-$ $\sigma) / \varepsilon^{2}$. Figure $5(\mathrm{~b})$ illustrates some PIGL demand manifolds. The best-known member of the PIGL family is the translog, $x(p)=\left(\gamma^{\prime}+\delta^{\prime} \log p\right) / p$, which is

\footnotetext{
${ }^{19} \mathrm{~A}$ third special case is the family of demand functions implied by the quadratic mean of order $r$ (QMOR) expenditure function introduced by Diewert (1976) and extended to monopolistic competition by Feenstra (2014). See Appendix B8 for details on the Pollak, PIGL and QMOR demand functions.

${ }^{20}$ Because $\nu$ and $\sigma$ enter symmetrically into $(13 \mathrm{~b})$, it is arbitrary which is set equal to zero. For concreteness and without loss of generality we assume $\delta \neq 0$ and $\sigma \neq 0$ throughout.

${ }^{21}$ To show that CARA demands are a special case, rewrite the constants as $\gamma=\gamma^{\prime}+\delta^{\prime} / \sigma$ and $\delta=-\delta^{\prime} / \sigma$, and apply l'Hôpital's Rule, which yields the CARA demand function $x=\gamma^{\prime}+\delta^{\prime} \log p, \delta^{\prime}<0$.

${ }^{22}$ These branches correspond to the same value of $\sigma$ but to different values of $\gamma$ and/or $\delta$, and so to different demand functions. No bipower demand function as defined in Proposition 3 can be subconvex for some values of output and superconvex for others. Recalling Figure 1(b), this is only possible if the manifold is horizontal where it crosses the superconvexity locus. Appendix B10 gives an example of a demand function, the inverse exponential, that exhibits this property.
} 
the limiting case as $\sigma$ approaches one so $\bar{\rho}(\varepsilon)=(3 \varepsilon-1) / \varepsilon^{2}{ }^{23}$ From the firm's perspective, this is consistent with both the Almost Ideal or "AIDS" model of Deaton and Muellbauer (1980), and the homothetic translog of Feenstra (2003). This class also includes Stone-Geary demands, the only case that is a member of both the Pollak and PIGL families (since $\nu=0$ and $\sigma=1$ are equivalent to $\nu=1$ and $\sigma=0$ in (13b)).

Just as the general bipower inverse and bipower direct demand functions in (13a) and (13b) are dual to each other, so also there are two important special cases of $(13 \mathrm{a})$ that are dual to the special cases of (13b) just considered. The first of these comes from setting $\eta$ in (13a) equal to zero, giving the inverse demand function $p(x)=\alpha+\beta x^{-\theta}$. This is the iso-convex or "constant passthrough" family of Bulow and Pfleiderer (1983), recently empirically implemented by Atkin and Donaldson (2012). The second important special case of (13a) comes from setting $\eta$ equal to one. This gives the "inverse PIGL" ("price-independent generalized linear") system, which is dual to the direct PIGL system considered earlier: expenditure $x p(x)$ is now a "translated-CES" function of sales: $p(x)=$ $\left(\alpha+\beta x^{1-\theta}\right) / x$. Further details about these demand functions and their manifolds are given in Appendix B9.

\section{E. Demand Manifolds and Comparative Statics}

It should be clear how the comparative statics implications of a given demand function can be illuminated by considering its demand manifold. To take a specific example, consider the Stone-Geary demand function (represented by the curves labeled $\sigma=1$ in Figure 5(a) and $\sigma=0$ in Figure 5(b)). Referring back to Figures 2 and 4 in Section I, we can conclude without the need for any calculations that Stone-Geary demands are always subconvex, and that they imply less than absolute pass-through and supermodular profits for small firms but the opposite for large ones. Inspecting the figures shows that, qualitatively, these properties are similar to those of the CARA and translog demand functions (except for a qualification in the latter case discussed in the next paragraph). However, they are quite different from those of the CES on the one hand or the linear demand function on the other.

Comparing demand functions in terms of their manifolds can also draw attention to hitherto unsuspected results. An example, which is suggested by Figure 5, is that the translog is the only bipower demand function that is both subconvex and supermodular throughout the admissible region: see the curve labeled $\sigma=1$ in Figure 5(b). We can go further and show that the translog is the only member of a broader class of demand functions, characterized in terms of their manifolds, that satisfies these conditions. We call the class in question a "contiguous bipower" manifold, since it expresses $\rho$ as a bipower function of $\varepsilon$, where the ex-

\footnotetext{
${ }^{23}$ To show this, rewrite the constants as $\gamma=\gamma^{\prime}-\delta^{\prime} /(1-\sigma)$ and $\delta=\delta^{\prime} /(1-\sigma)$, and apply l'Hôpital's Rule, which yields the translog demand function.
} 
ponents are contiguous integers, $\kappa$ and $\kappa+1$; this includes both bipower direct and bipower inverse demands, for which $\kappa$ equals -2 and zero, respectively: ${ }^{24}$

LEMMA 1: The translog is the only demand function with a contiguous bipower manifold, $\rho=a_{1} \varepsilon^{\kappa}+a_{2} \varepsilon^{\kappa+1}$, where $\kappa$ is an integer, that is always both strictly subconvex and strictly supermodular in the interior of the admissible region.

This is an attractive feature: the translog is the only demand function from a very broad family that allows for competition effects (so markups fall with globalization) but also implies that larger firms always serve foreign markets via FDI rather than exports.

Yet another use of the demand manifold is to back out demand functions with desirable properties. As an example, recall the discussion of proportional pass-through in Section I.C. We saw there that the elasticity of pass-through, $d \log p / d \log c$, is constant and equal to $k(k>0)$ if and only if equation (6) holds. We can now see that this equation defines a family of demand manifolds for different values of $k$, as illustrated in Figure 2(a). Integrating it gives the implied demand function, which we call "CEPT" for "Constant Elasticity of Pass-Through" : ${ }^{25}$

$$
p(x)=\frac{\beta}{x}\left(x^{\frac{k-1}{k}}+\gamma\right)^{\frac{k}{k-1}}
$$

This demand function appears to be new and is likely to have many uses in applied work. We will give an illustration in Section IV.A below. In the special case where $k=1 / 2$, the CEPT demand function is identical to the Stone-Geary, both with manifolds given by $\rho=2 / \varepsilon$. This yields the result that, with Stone-Geary demands, all firms pass through exactly $50 \%$ of cost increases to consumers.

\section{F. Demand Functions that are Not Manifold-Invariant}

In the rest of the paper we concentrate on the demand functions introduced here which have manifolds that are invariant with respect to at least some parameters. However, even in more complex cases when the demand manifold has the same number of parameters as the demand function, it typically provides an economy of information by highlighting which parameters matter for comparative statics. Appendix B12 presents two examples of this kind that nest some important cases, such as the "Adjustable Pass-Through" (APT) demand function of Fabinger and Weyl (2012).

\footnotetext{
${ }^{24}$ The proof is in Appendix B11.

25 "CEPT" is pronounced as in "September". Equation (14) is the solution to (6) when $k \neq 1$. When $k=1,(6)$ becomes $\rho=(\varepsilon+1) / \varepsilon$, which, as we saw in Section I.B, defines the family of point-manifolds for the CES case. Note that the CEPT manifold is a member of the contiguous bipower class, with $\kappa$ equal to -1 , so Lemma 1 applies. See Appendix A3 for further details on the CEPT demand function.
} 


\section{Monopolistic Competition in General Equilibrium}

To illustrate the power of the approach we have developed in previous sections, we turn next to apply it to a canonical model of international trade, a one-sector, one-factor, multi-country, general-equilibrium model of monopolistic competition, where countries are symmetric and trade is unrestricted. ${ }^{26}$ Following Krugman (1979) and a large subsequent literature, we model globalization as an increase in the number of countries in the world economy. On the consumer side, we assume that preferences are symmetric, and that the elasticity of demand for a good depends only on the level of consumption of that good. From Goldman and Uzawa (1964), this is equivalent to assuming additively separable preferences as in Dixit and Stiglitz (1977) and Krugman (1979):

$$
U=F\left[\int_{0}^{N} u\{x(\omega)\} \mathrm{d} \omega\right], F^{\prime}>0, u^{\prime}(x)>0, u^{\prime \prime}(x)<0
$$

We begin with the effects of globalization on industry equilibrium, first in Section III.A with homogeneous firms as in Krugman (1979), and then in Section III.B with heterogeneous firms as in Melitz (2003). Finally in Section III.C we look at the effects of globalization on welfare.

\section{A. Globalization with Homogeneous Firms}

Symmetric demands and homogeneous firms imply that we can dispense with firm subscripts from the outset. Industry equilibrium requires that firms maximize profits by choosing output $y$ to set marginal revenue MR equal to marginal cost $\mathrm{MC}$, and that profits are driven to zero by free entry (so average revenue AR equals average cost $\mathrm{AC})$ :

$$
\begin{aligned}
\text { Profit Maximization }(\mathrm{MR}=\mathrm{MC}): & p=\frac{\varepsilon(x)}{\varepsilon(x)-1} c \\
\text { Free Entry }(\mathrm{AR}=\mathrm{AC}): & p=\frac{f}{y}+c
\end{aligned}
$$

The model is completed by market-clearing conditions for the goods and labor markets:

$$
\begin{array}{lll}
\text { Goods-Market Equilibrium (GME): } & & y=k L x \\
\text { Labor-Market Equilibrium (LME): } & & L=n(f+c y)
\end{array}
$$

Here $L$ is the number of worker/consumers in each country, each of whom supplies one unit of labor and consumes an amount $x$ of every variety; $k$ is the number of identical countries; and $n$ is the number of identical firms in each, all with total

\footnotetext{
${ }^{26}$ The effects of changes in trade costs are considered in Mrázová and Neary (2014).
} 
output $y$, so $N=k n$ is the total number of firms in the world. Since all firms are single-product by assumption, $N$ is also the total number of varieties available to all consumers.

Equations (16) to (19) comprise a system of four equations in four endogenous variables, $p, x, y$ and $n$, with the wage rate set equal to one by choice of numéraire. To solve for the effects of globalization, an increase in the number of countries $k$, we totally differentiate the equations, using "hats" to denote logarithmic derivatives, so $\hat{x} \equiv d \log x, x \neq 0$ :

$$
\begin{aligned}
\mathrm{MR}=\mathrm{MC}: & \hat{p}=\frac{\varepsilon+1-\varepsilon \rho}{\varepsilon(\varepsilon-1)} \hat{x} \\
\mathrm{AR}=\mathrm{AC}: & \hat{p}=-(1-\omega) \hat{y} \\
\mathrm{GME}: & \hat{y}=\hat{k}+\hat{x} \\
\mathrm{LME}: & 0=\hat{n}+\omega \hat{y}
\end{aligned}
$$

Consider first the $\mathrm{MR}=\mathrm{MC}$ equilibrium condition, equation (20). Clearly $p$ and $x$ move together if and only if $\varepsilon+1-\varepsilon \rho>0$, i.e., if and only if demand is subconvex. This reflects the property noted in Section I.B: higher sales are associated with a higher proportional markup, $p / c$, if and only if they imply a lower elasticity of demand. As for the free-entry condition, equation (21), it shows that the fall in price required to maintain zero profits following an increase in firm output is greater the smaller is $\omega \equiv c y /(f+c y)$, the share of variable in total costs, which is an inverse measure of returns to scale. This looks like a new parameter but in equilibrium it is not. It equals the ratio of marginal cost to price, $c / p$, which because of profit maximization equals the ratio of marginal revenue to price $\left(p+x p^{\prime}\right) / p$, which in turn is a monotonically increasing transformation of the elasticity of demand $\varepsilon: \omega=c / p=\left(p+x p^{\prime}\right) / p=(\varepsilon-1) / \varepsilon$. Similarly, equation (23) shows that the fall in the number of firms required to maintain full employment following an increase in firm output is greater the larger is $\omega$. It follows by inspection that all four equations depend only on two parameters, which implies:

LEMMA 2: With additive separability, the local comparative statics responses of the symmetric monopolistic competition model to a globalization shock depend only on $\varepsilon$ and $\rho$.

Lemma 2 implies that the comparative statics results can be directly related to the demand manifold. To see this in detail, solve for the effects of globalization on outputs, prices and the number of firms in each country:

$$
\hat{y}=\frac{\varepsilon+1-\varepsilon \rho}{\varepsilon(2-\rho)} \hat{k}, \quad \hat{p}=-\frac{1}{\varepsilon} \hat{y}=-\frac{\varepsilon+1-\varepsilon \rho}{\varepsilon^{2}(2-\rho)} \hat{k}, \quad \hat{n}=-\frac{\varepsilon-1}{\varepsilon} \hat{y}
$$

(Details of the solution are given in Appendix B13.) The signs of these depend solely on whether demands are sub- or superconvex, i.e., whether $\varepsilon+1-\varepsilon \rho$ 
is positive or negative. With subconvexity we get what Krugman (1979) called "sensible" results: globalization prompts a shift from the extensive to the intensive margin, with fewer but larger firms in each country, as firms move down their average cost curves and prices of all varieties fall. With superconvexity, all these results are reversed. ${ }^{27}$ The CES case, where $\varepsilon+1-\varepsilon \rho=0$, is the boundary one, with firm outputs, prices, and the number of firms per country unchanged. The only effects that hold irrespective of the form of demand are that consumption per head of each variety falls and the total number of varieties produced in the world and consumed in each country rises:

$$
\hat{x}=-\frac{1}{2-\rho} \frac{\varepsilon-1}{\varepsilon} \hat{k}<0, \quad \hat{N}=\frac{(\varepsilon-1)^{2}+(2-\rho) \varepsilon}{\varepsilon^{2}(2-\rho)} \hat{k}>0
$$

Qualitatively these results are not new. The new feature that our approach highlights is that their quantitative magnitudes depend only on two parameters, $\varepsilon$ and $\rho$, the same ones on which we have focused throughout. Hence the results in (24) and (25) can be directly related to the demand manifold from Section II.

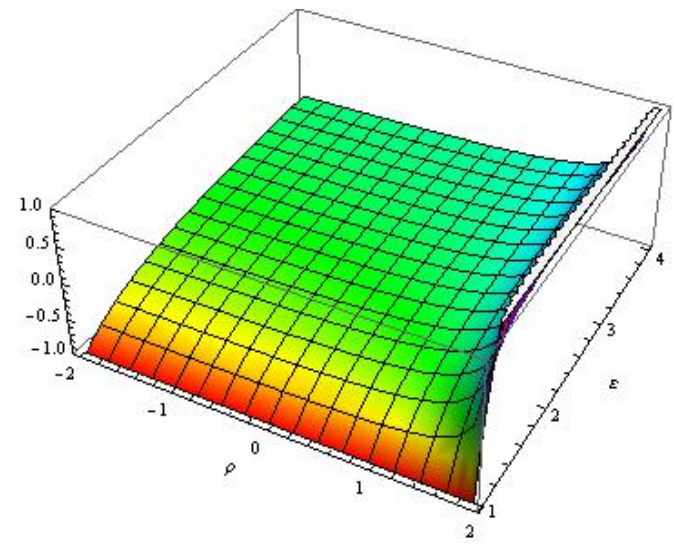

(a) Changes in Prices

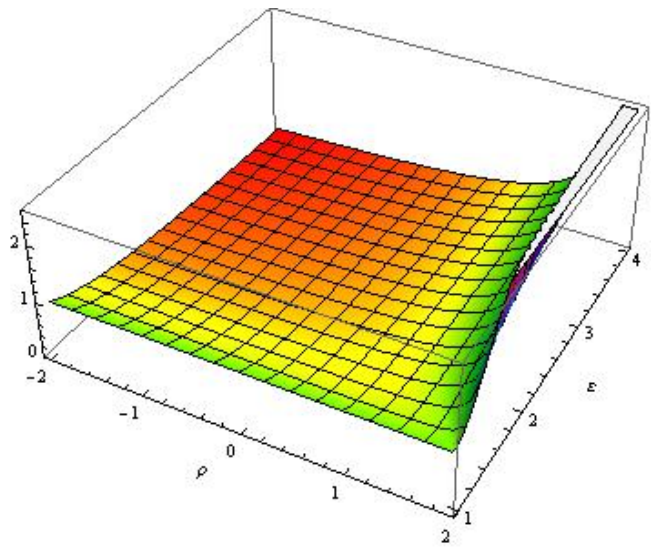

(b) Changes in the Number of Varieties

Figure 6. The Effects of Globalization

To illustrate how this works, Figure 6 gives the quantitative magnitudes of changes in the two variables that matter most for welfare: prices and the number of varieties. In each panel, the vertical axis measures the proportional change in either $p$ or $N$ following a unit increase in $k$ as a function of the elasticity and convexity of demand. The three-dimensional surfaces shown are independent of the functional form of demand, so we can combine them with the results on demand manifolds from Section II to read off the quantitative effects of globalization implied by different assumptions about demand. We know already from equations

\footnotetext{
${ }^{27}$ See Neary (2009) and Zhelobodko et al. (2012).
} 
(24) and (25) that prices fall if and only if demand is subconvex and that product variety always rises. The figures show in addition that less elastic demand implies greater falls in prices and larger increases in variety, except when demand is highly convex; ${ }^{28}$ while more convex demand always implies greater increases in both prices and variety.

To summarize this sub-section, Lemma 2 implies that the demand manifold is a sufficient statistic for the effects of globalization on industry equilibrium in the Krugman (1979) model, just as it is for the comparative statics results discussed in Section I. Moreover, as in Section II.E, given a particular demand function, we can immediately infer its implications for the comparative statics of globalization by combining its demand manifold with Figure 6 .

\section{B. Heterogeneous Firms}

The case of homogeneous firms is of independent interest, and also provides a key reference point for understanding the comparative statics of a model with heterogeneous firms and general demands. Consider the same model as before, except that now firms differ in their marginal costs $c$, which, as in Melitz (2003), are given by an exogenous distribution $g(c)$, with support on $[\underline{c}, \bar{c}]$. The maximum operating profit that a firm can earn varies inversely with its own marginal cost $c$. Through the inverse demand function $p(y, \lambda, k)$, it also depends negatively on the marginal utility of income, $\lambda$, and positively on the size of the global economy $k$ :

$$
\pi(\underset{c}{c}, \underset{-}{\lambda}, \underset{+}{k}) \equiv \max _{y}[p(y, \lambda, k)-c] y
$$

A key implication of this specification is that, in monopolistic competition, where individual firms are infinitesimal relative to the industry, $\lambda$ is endogenous to the industry, but exogenous to firms, and so can be interpreted as a measure of the degree of competition each firm faces. ${ }^{29}$

With homogeneous firms, equation (17) in Section III.A gives a free-entry condition that is common to all firms. With heterogeneous firms, this must be replaced by two conditions. First is the zero-profit condition for cutoff firms, which requires that their operating profits equal the common fixed cost $f$ :

$$
\pi\left(c_{0}, \lambda, k\right)=f
$$

This determines the cutoff $\operatorname{cost} c_{0}$ as a function of $\lambda$ and $k$. Second is the zeroexpected-profit condition for all firms. A potential entrant bases its entry decision on the value $v(c, \lambda, k)$ that it expects to earn; firm value is zero for firms that get a

\footnotetext{
${ }^{28} \hat{p} / \hat{k}$ is increasing in $\varepsilon$ if and only if $\rho<1+2 / \varepsilon$, and $\hat{N} / \hat{k}$ is decreasing in $\varepsilon$ if and only if $\rho<2 / \varepsilon$.

${ }^{29}$ This specification is also consistent with a much broader class of preferences than additive separability, which Pollak (1972) calls "generalized additive separability". See Mrázová and Neary (2018) for further discussion.
} 
high-cost draw and equals operating profits less fixed costs otherwise. Equilibrium requires that the expected value of a firm, $\bar{v}(\lambda, k)$, equal the sunk cost of entering the industry $f_{e}$ :

$$
\bar{v}(\lambda, k) \equiv \int_{\underline{c}}^{\bar{c}} v(c, \lambda, k) g(c) \mathrm{d} c=f_{e}, \quad \text { where } \quad v(c, \lambda, k) \equiv \max [0, \pi(c, \lambda, k)-f]
$$

Expected profits are conditional on incurring the sunk cost of entry, not conditional on actually entering, and so they do not depend directly on the cutoff $c_{0}$. Equation (28) thus determines the level of competition as a function of the size of the world economy $k$.

We can now derive the effects of globalization on the profile of profits across firms. Combining the profit function and equation (28) gives:

$$
\hat{\pi}=\frac{k \pi_{k}}{\pi} \hat{k}+\frac{\lambda \pi_{\lambda}}{\pi} \hat{\lambda}=(\underbrace{\frac{k \pi_{k}}{\pi}}_{(M)}-\underbrace{\frac{\lambda \pi_{\lambda}}{\pi} \frac{\bar{v}}{\lambda \bar{v}_{\lambda}} \frac{k \bar{v}_{k}}{\bar{v}}}_{(C)}) \hat{k}
$$

This shows that globalization has a market-size effect, given by $(\mathrm{M})$, which tends to raise each firm's profits. In addition it has a competition effect, given by $(\mathrm{C})$ : because all firms' profits rise at the initial level of competition, the latter must increase to ensure that expected profits remain equal to the fixed cost of entry; this in turn tends to reduce each firm's profits. The net outcome is indeterminate in general. However, with additive separability, equation (29) takes a particularly simple form (see Appendix A4 for details):

$$
\hat{\pi}=\left(1-\frac{\varepsilon}{\bar{\varepsilon}}\right) \hat{k} \quad \text { where } \quad \bar{\varepsilon} \equiv \int_{\underline{c}}^{\bar{c}} \frac{v(c, \lambda, k)}{\bar{v}(\lambda, k)} \varepsilon(c) g(c) \mathrm{d} c
$$

Here $\bar{\varepsilon}$ is the profit-weighted average elasticity of demand across all firms, which we can interpret as the elasticity faced by the average firm. Thus the market-size effect is one-for-one (given $\lambda$, all firms' profits increase proportionally with $k$ ), while the competition effect is greater than one if and only the elasticity a firm faces is greater than the average elasticity. The implications for the response of profits across firms are immediate, recalling that firms face an elasticity of demand that falls with their output if and only if demands are subconvex:

PROPOSITION 5: With additive separability, globalization pivots the profile of profits across firms around the average firm; if and only if demands are subconvex, profits rise for firms above the average, and by more the larger a firm's initial sales.

As in Section 4.1 of Melitz (2003), globalization leaves the profits of all firms unchanged in the CES case (where $\varepsilon=\bar{\varepsilon}=\sigma$ for all firms). By contrast, in the realistic case when demand is subconvex, the elasticity of demand is smaller for 


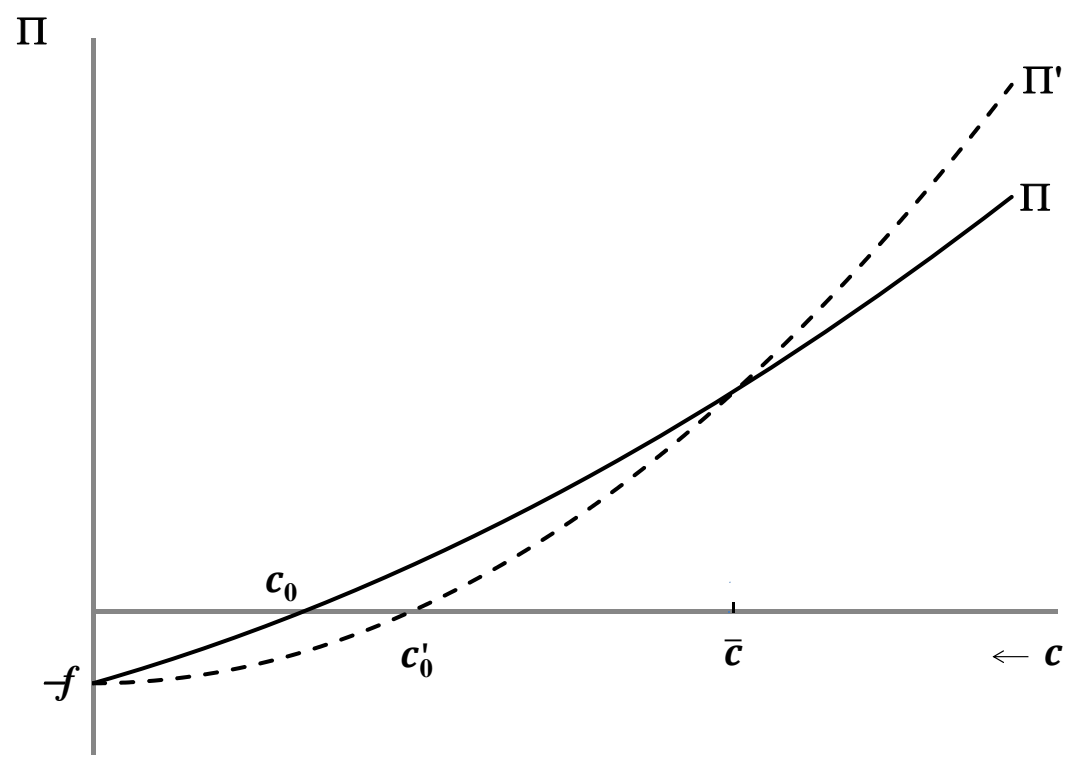

Figure 7. Effects of Globalization on Firm Profits and Selection with Subconvex Demand

firms with above-average output, and so the outcome exhibits a strong "Matthew Effect" ("to those who have, more shall be given"). This is illustrated in Figure 7, where the solid locus $\Pi$ denotes the initial profile of profits across firms, while the dashed locus $\Pi^{\prime}$ denotes the post-globalization profile when demand is subconvex. ${ }^{30}$ The market-size effect dominates for larger firms, so they expand; the competition effect dominates for smaller firms, so they contract, and some (those at or just to the right of the initial cutoff cost level $c_{0}$ ) exit; ${ }^{31}$ as a result, the average productivity of active exporters rises. All these results are reversed when demands are superconvex: now larger firms face higher elasticities of demand, so their profits fall, whereas those of smaller firms rise, and globalization encourages entry of less efficient firms.

In the same way we can solve for the effects of globalization on the intensive margin. As shown in Appendix A4, the changes in the profiles of firm outputs

\footnotetext{
${ }^{30}$ Marginal cost $c$ is increasing from right to left along the horizontal axis. It can be checked that profits are decreasing and convex in $c$.

${ }^{31}$ To solve for the effect of globalization on the extensive margin, we can use (30) to evaluate the change in the cutoff marginal cost defined by $(27): \hat{c}_{0} / \hat{k}=\left(1-\varepsilon_{0} / \bar{\varepsilon}\right) /\left(\varepsilon_{0}-1\right)$, where $\varepsilon_{0} \equiv \varepsilon\left(c_{0}\right)$ is the elasticity faced by firms at the cutoff. Such firms have the lowest sales of all active firms and so, when demands are subconvex, they face the highest elasticity: $\varepsilon_{0}>\bar{\varepsilon}$. Hence the competition effect dominates and the least efficient firms exit.
} 
and prices are given by:

$$
\begin{aligned}
& \hat{y}=[\underbrace{\frac{\bar{\varepsilon}+1-\bar{\varepsilon} \bar{\rho}}{\bar{\varepsilon}(2-\bar{\rho})}}_{*}+\frac{1}{\bar{\varepsilon}}\left(\frac{\bar{\varepsilon}-1}{2-\bar{\rho}}-\frac{\varepsilon-1}{2-\rho}\right)] \hat{k}, \\
& \hat{p}=[\underbrace{-\frac{\bar{\varepsilon}+1-\bar{\varepsilon} \bar{\rho}}{\bar{\varepsilon}^{2}(2-\bar{\rho})}}_{*}-\frac{1}{\bar{\varepsilon}}\left(\frac{\bar{\varepsilon}-1}{\bar{\varepsilon}(2-\bar{\rho})}-\frac{\varepsilon-1}{\varepsilon(2-\rho)}\right)] \hat{k}
\end{aligned}
$$

These changes in output and price for each firm have two components. The first, denoted by $*$, equals the change for the average firm, which is the same as the change for all firms in the homogeneous-firms case (given by (24)). Hence, for the average firm, output rises and price falls if and only if the demand it faces is subconvex. Figure 6(a) therefore illustrates the change in the average firm's price, so, as in Section III.A, we can evaluate this by combining the figure with the appropriate demand manifold. The second component is a correction factor that adjusts for the differences between the individual firm and the average firm. Its sign depends on the difference between both the elasticity and convexity of the individual firm and those of the average firm. For example, if demand is subconvex, then outputs of above-average firms tend to rise relative to the average firm, and to rise by more the larger the firm; while outputs of below-average firms tend to fall relative to the average firm, and to fall by more the smaller the firm. ${ }^{32}$ Similar considerations apply to the change in prices.

\section{Globalization and Welfare}

The final application of the manifold we consider is to the effects of globalization on welfare. It is clear that the demand parameters summarized by the manifold are an important component of calculating the gains from globalization, but it is also clear that they cannot be a sufficient statistic for welfare change in general. At the very least, if firms are heterogeneous, we also need to know one or more parameters of the productivity distribution. However, the manifold is a sufficient statistic for the gains from trade in some cases: specifically, when the distribution of firm productivities is degenerate, so firms are homogeneous, and when the functional form of the sub-utility function is restricted in ways to be explained below. So, to highlight the role of the manifold, we return in this sub-section to the case of homogeneous firms as in Section III.A. As a benchmark, this case is of great interest in itself. It also gives a lower bound to the gains from trade in an

\footnotetext{
${ }^{32}$ Recalling footnote 15 , the correction factor for outputs depends on the difference between the inverse elasticity of marginal revenue of the individual firm and that of the average firm. The exact condition for the change in output with globalization to be increasing in firm size is: $(2-\rho) \varepsilon_{y}+(\varepsilon-1) \rho_{y}<0$. When demand is subconvex (so $\varepsilon_{y}<0$ ), this condition holds for almost all the demand functions discussed in Section II.D, including all members of the PIGL and Bulow-Pfleiderer families (trivially for the latter since $\rho_{y}=0$ ), and almost all members of the Pollak family. However, this tendency could be reversed if $\rho_{y}$ were positive and sufficiently large.
} 
otherwise identical model with firm heterogeneity, at least with CES preferences, as shown empirically and theoretically by Balistreri, Hillberry and Rutherford (2011) and Melitz and Redding (2015). ${ }^{33}$

To quantify the welfare effects of globalization, we assume as in previous sections that preferences are additively separable. With homogeneous firms, symmetric preferences, and no trade costs, the overall utility function (15) becomes: $U=$ $F[N u(x)]$. So, welfare depends on the extensive margin of consumption $N$ times the utility of the intensive margin $x$. Using the budget constraint to eliminate $x$, we can write the change in utility in terms of its income equivalent $\hat{Y}$ (see Appendix B14 for details):

$$
\hat{Y}=\frac{1-\xi}{\xi} \hat{N}-\hat{p}
$$

Here $\xi(x) \equiv x u^{\prime}(x) / u(x)$ is the elasticity of the sub-utility function $u(x)$ with respect to consumption. We thus have a clear division of roles between three preference parameters: on the one hand, as we saw in Section III.A, $\varepsilon$ and $\rho$ determine the effects of globalization on the two variables, number of varieties, $N$, and prices, $p$, that affect consumers directly; on the other hand, $\xi$ determines the relative importance of $N$ and $p$ in affecting welfare. It is clear from (33) that $\xi$ must lie between zero and one if preferences exhibit a taste for variety. (See also Vives (1999).) Moreover, $\xi$ is an inverse measure of preference for variety, since welfare rises more slowly with $N$ the higher is $\xi$.

Next, we can substitute for the changes in prices and number of varieties from equations (24) and (25) in Section III.A into (33) to obtain an explicit expression for the gain in welfare in terms of preference and demand parameters only:

$$
\hat{Y}=\frac{1}{\xi \varepsilon}\left[1-\left(\xi-\frac{\varepsilon-1}{\varepsilon}\right) \frac{\varepsilon-1}{2-\rho}\right] \hat{k}
$$

Now there are three sufficient statistics for the change in welfare, only one of which has an unambiguous effect. The gains from globalization are always decreasing in $\xi$ : unsurprisingly, consumers gain more from a proliferation of countries and hence of products, the greater their taste for variety. By contrast, the gains from globalization depend ambiguously on both $\varepsilon$ and $\rho$. Of course, the values of the three key parameters do not in general vary independently of each other, but without further assumptions we cannot say much about how they vary together.

One case where equation (34) simplifies dramatically is when preferences are CES, so $u(x)=(\sigma /(\sigma-1)) \beta x^{(\sigma-1) / \sigma}$. Now the elasticity of utility $\xi$ equals $(\sigma-1) / \sigma$, while $\varepsilon$ and $\rho$ equal $\sigma$ and $(\sigma-1) / \sigma$ respectively, as we have already seen

\footnotetext{
${ }^{33}$ By "otherwise identical" we mean with the same structural parameters except a non-degenerate distribution of firm productivities. If instead the comparison is carried out holding constant the elasticity of trade, then the gains from trade are the same in homogeneous and heterogeneous firms models as shown by Arkolakis, Costinot and Rodríguez-Clare (2012).
} 
in Section I.B. Substituting these values into (34), the gains from globalization, $\hat{Y} / \hat{k}$, reduce to $1 /(\sigma-1)$, exactly the expression found for the gains from trade in a range of CES-based models by Arkolakis, Costinot and Rodríguez-Clare (2012).

The key feature of the CES case is that the elasticities of utility and demand are directly related, without the need to specify any parameters. To move beyond the CES case, we would like to be able to express the elasticity of utility $\xi$ as a function of $\varepsilon$ and $\rho$ only. If this function is independent of parameters, then we can locate equation $(34)$ in $(\varepsilon, \rho)$ space, and use the results of Section II to relate it to the underlying demand function. To see when this can be done, recall that the demand manifold relates $\varepsilon, \rho$, and the non-invariant parameter $\phi$, which in general is vector-valued. To this can be added a second equation, which we call the "utility manifold", that relates $\xi, \varepsilon$, and $\phi .{ }^{34}$ We thus have two equations in $3+m$ unknowns, where $m$ is the dimension of $\phi$. Clearly, the demand manifold, and the space of $\{\varepsilon, \rho\}$ that it highlights, is particularly useful when $m$ equals one, since then we can eliminate $\phi$. In that case we can express both the elasticity of utility, $\xi$, and hence, using (34), the gains from globalization, $\hat{Y} / \hat{k}$, as functions of $\varepsilon$ and $\rho$ only. We can summarize these results in a way that brings out the parallel with Lemma 2 in Section III.A:

LEMMA 3: With additive separability, the gains from globalization in the symmetric monopolistic competition model depend only on $\varepsilon$ and $\rho$ if and only if $\phi$, the vector of non-invariant parameters in the utility and demand manifolds, is of dimension less than or equal to one.

To see the usefulness of this, we consider two of the families of demand functions discussed in Section II, whose manifolds depend on only a scalar non-invariant parameter.

\section{Globalization and Welfare with Bulow-Pfleiderer Preferences}

The first example we consider is that of Bulow-Pfleiderer demands, given by the demand function (13a) in Section II.D. Assuming that preferences are additively separable, we can integrate that function to obtain the corresponding sub-utility function, which also takes a bipower form: ${ }^{35}$

$$
u(x)=\alpha x+\frac{1}{1-\theta} \beta x^{1-\theta}
$$

\footnotetext{
${ }^{34}$ In an earlier version of this paper, Mrázová and Neary (2013), we gave more details of this equation and its geometric representation. Its derivation parallels that of the demand manifold. Recall that the demand manifold is derived by eliminating consumption $x$ from the expressions for the elasticity and curvature of the demand function, $\varepsilon(x, \phi)$ and $\rho(x, \phi)$, to obtain a smooth curve in $(\varepsilon, \rho)$ space. In the same way, eliminating $x$ from the expressions for the elasticity and curvature of the sub-utility function, $\xi(x, \phi)$ and $\varepsilon(x, \phi)$, yields a smooth curve in $\{\xi, \varepsilon\}$ space, whose properties are analogous to those of the demand manifold.

${ }^{35}$ It is natural to set the constant of integration to zero, which implies that $u(0)=0$. We return to this issue in the next sub-section.
} 
From this we can calculate the utility manifold, which gives the elasticity of

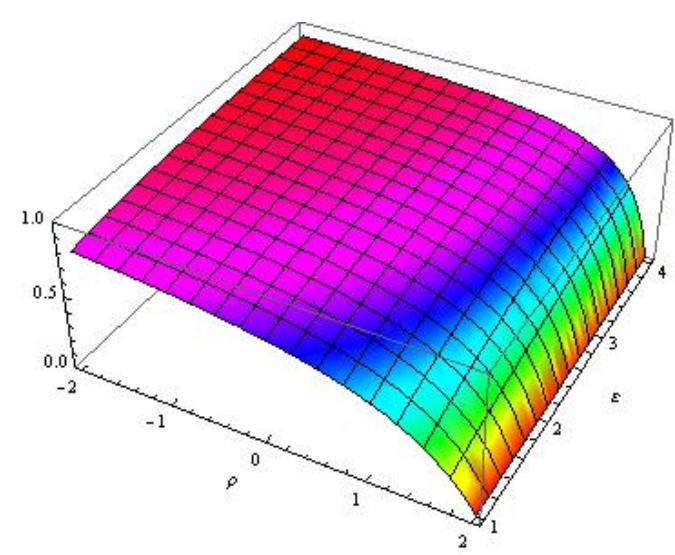

(a) Elasticity of Utility

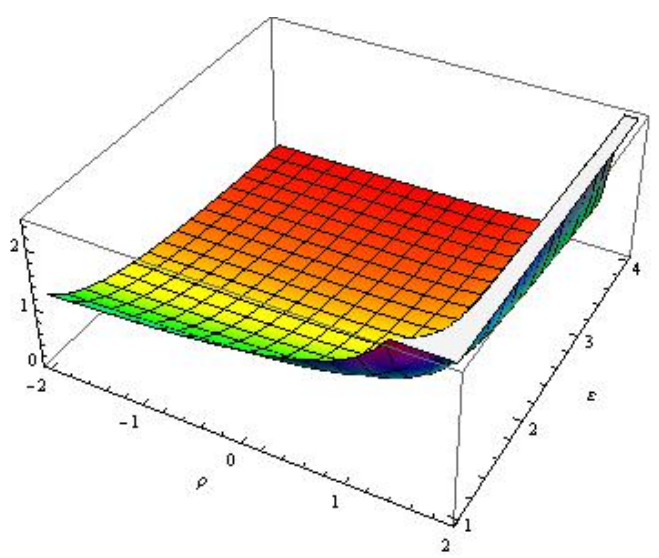

(b) Change in Real Income

Figure 8. Globalization and Welfare: Bulow-Pfleiderer Preferences

utility $\xi$ as a function of $\varepsilon$ and the non-invariant parameter $\theta$, and then use the demand manifold from Section II.D, $\rho=\theta+1$, to solve for $\xi$ as a function of $\varepsilon$ and $\rho$ (details are in Appendix B16):

$$
\xi(\varepsilon, \theta)=\frac{(1-\theta) \varepsilon}{(1-\theta) \varepsilon+1} \quad \Rightarrow \quad \xi(\varepsilon, \rho)=\frac{(2-\rho) \varepsilon}{(2-\rho) \varepsilon+1}
$$

Clearly $\xi$ always lies between zero and one when $\varepsilon$ and $\rho$ are in the admissible region. Figure $8(\mathrm{a})$ illustrates the second of these expressions, while substituting into equation (34) allows us to express the change in real income as a function of $\varepsilon$ and $\rho$ only, as illustrated in Figure 8(b). As panel (a) illustrates, the elasticity of utility is increasing in the elasticity of demand, and decreasing in convexity: there is a greater taste for variety at high $\rho$; while panel (b) shows that the gains from globalization are always positive, are decreasing in $\varepsilon$, and increasing in $\rho$.

\section{Globalization and Welfare with Pollak Preferences}

The second example we consider is the Pollak demand function from Section II.D. The welfare implications of this specification are sensitive to how we normalize the sub-utility function. To highlight the contrast with the BulowPfleiderer case in the previous sub-section, we focus in the text on the case considered by Pollak (1971) and Dixit and Stiglitz (1977). This derives the sub-utility function from the demand function without imposing a constant of integration, implying that $u(0)$ is non-zero: consumers gain from the presence of more varieties even if they do not consume them, an outcome whose plausibility was defended 
by Dixit and Stiglitz (1979). ${ }^{36}$ This gives the following sub-utility function:

$$
u(x)=\frac{\beta}{\sigma-1}(\sigma x+\zeta)^{\frac{\sigma-1}{\sigma}}
$$

Relative to the Pollak demand function in Section II.D, it is convenient to redefine the constants as $\zeta \equiv-\gamma \sigma$ and $\beta \equiv(\delta / \sigma)^{1 / \sigma}$. (See Appendix B16 for details.)

As in the Bulow-Pfleiderer case, we can calculate the utility manifold, giving $\xi$ as a function of $\varepsilon$ and the non-invariant parameter $\sigma$, and then use the demand manifold from Section II.D, $\bar{\rho}(\varepsilon)=(\sigma+1) / \varepsilon$, to solve for $\xi$ as a function of $\varepsilon$ and $\rho:$

$$
\xi(\varepsilon, \sigma)=\frac{\sigma-1}{\varepsilon} \Rightarrow \xi(\varepsilon, \rho)=\frac{\varepsilon \rho-2}{\varepsilon}
$$

Since only values of $\xi$ between zero and one are consistent with a preference for variety, we restrict attention to the range $2 / \varepsilon<\rho<1+2 / \varepsilon^{37}$ Within this range, the behavior of the elasticity of utility is the opposite to that in the Bulow-Pfleiderer case. ${ }^{38}$ Figure 9 illustrates how the elasticity of utility and the

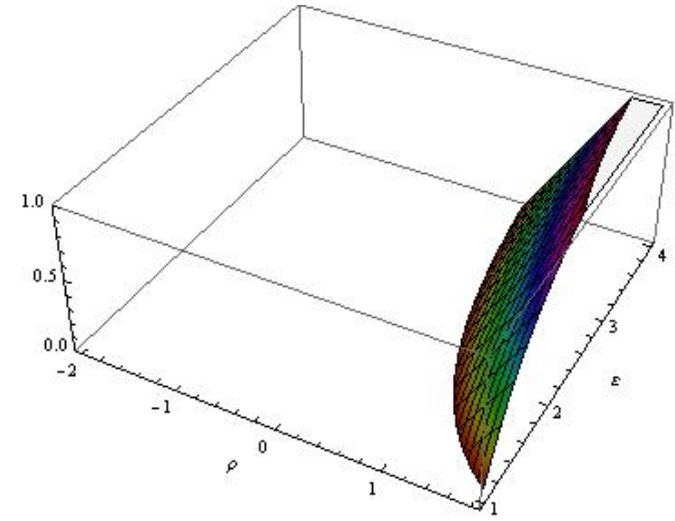

(a) Elasticity of Utility

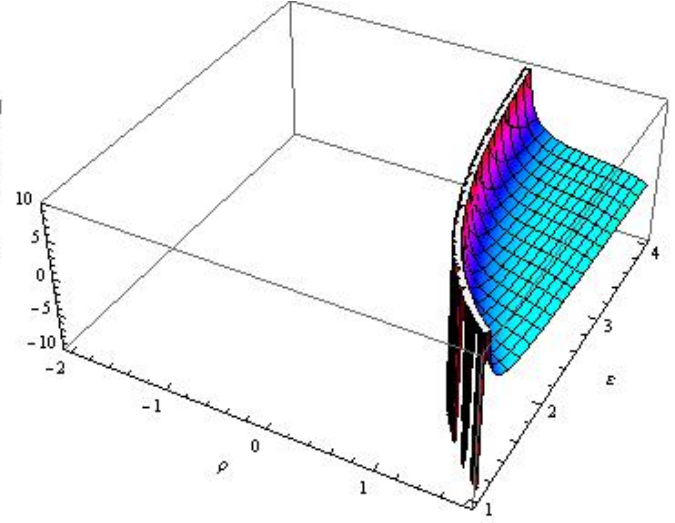

(b) Change in Real Income

Figure 9. Globalization and Welfare: Pollak Preferences

gains from globalization vary with $\varepsilon$ and $\rho$ in this case. The contrast with the Bulow-Pfleiderer case in Figure 8 could hardly be more striking. Panel (a) shows that the elasticity of utility is now increasing in both $\varepsilon$ and $\rho$ : consumers have a

\footnotetext{
${ }^{36}$ In Appendix B16 we consider an alternative specification, due to Pettengill (1979), which imposes the restriction that $u(0)=0$, and yields different results.

${ }^{37}$ See Pettengill (1979) and Dixit and Stiglitz (1979). This range has the Linear Expenditure System $(\rho=2 / \varepsilon)$ as its lower bound, and it includes demand functions that are both sub- and superconvex $(\rho \underset{38}{\lessgtr}(\varepsilon+1) / \varepsilon)$.

38 This extends a result of Vives (1999), p. 371.
} 
lower taste for variety at high $\rho$, which we know from Figure 6(a) is when prices increase most. As a result, welfare can fall with globalization. As panel (b) shows, the gains from globalization are decreasing in both $\varepsilon$ and $\rho$, and are negative for sufficiently convex demand: as shown in Appendix B16, the exact condition for this is $\rho>\left(\varepsilon^{2}+2 \varepsilon-1\right) / \varepsilon^{2}$. This provides, to our knowledge, the first concrete example of the "folk theorem" that globalization in the presence of monopolistic competition can be immiserizing if demand is sufficiently superconvex. Perhaps equally striking is that welfare rises by more for lower values of $\varepsilon$ and $\rho$ : estimates based on CES preferences grossly underestimate the gains from globalization in much of the subconvex region, just as they fail to predict losses from globalization in the superconvex region.

\section{Empirically Applying the Demand Manifold}

So far, we have presented a theoretical framework that highlights the elasticity and convexity of demand as sufficient statistics for a broad class of theoretical results, and shown how they are related to each other via the demand manifold implied by the underlying demand function. Next, we want to illustrate some potential empirical applications of the manifold. We continue to assume, as in previous sections, that the observations come from a monopolistically competitive industry with constant marginal costs. Section IV.A shows how, with no further assumptions, an empirical manifold can be estimated if we have information on markups and pass-through coefficients for a sample of firms. Section IV.B shows how this empirical manifold can be used to infer income elasticities if we assume in addition that consumer preferences are additively separable. Finally, Section IV.C shows how we can go further and use the manifold for welfare analysis if we are willing to make further parametric assumptions about the structure of preferences and the distribution of firm productivities.

\section{A. Estimating the Manifold}

Clearly, if we have estimates of a demand function we can directly calculate an empirical manifold. However, this requires taking a stand on the parametric form of demand. An alternative approach is to draw on recently-developed methods that make it possible to proceed without making parametric assumptions about preferences or demand. In particular, the methods developed by Jan De Loecker, Pinelopi K Goldberg, Amit K Khandelwal and Nina Pavcnik (2016) for inferring markups and estimating pass-through coefficients do not impose assumptions about the form of demand or the underlying market structure.

Suppose therefore that we have a data set giving information on markups and pass-through coefficients for a sample of firms. In order to apply our approach from previous sections, we need to assume that the observations are generated by a monopolistically competitive industry, with constant marginal costs. Given these assumptions (though without any restrictions on preferences or the distribution of productivities), we can invoke from Section I.C the expressions for the 
proportional markup $m$ and the proportional pass-through coefficient $k$, which we repeat here for convenience:

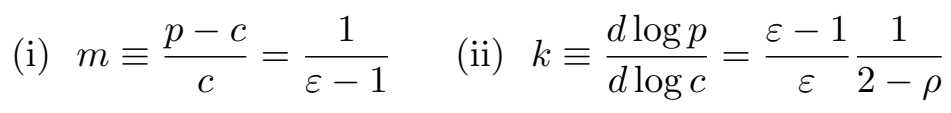

Using these, it is straightforward to back out the values of the elasticity and convexity, provided we know (or have estimates of) the markup and the passthrough coefficient:

$$
\begin{array}{ll}
\text { (i) } \varepsilon=\frac{m+1}{m} & \text { (ii) } \rho=2-\frac{1}{k} \frac{1}{m+1}
\end{array}
$$

This gives a two-dimensional array that can be represented by a "cloud" of points in $(\varepsilon, \rho)$ space.

The next step is to infer from these data an underlying demand manifold. In effect, this means that we want to estimate the parametric manifold that gives the best fit to the data in some sense. This does not mean that we need to assume a particular form of demand itself: as discussed in Section II, we can expect the manifold to be invariant with respect to some demand parameters in most cases. Hence estimating the manifold requires inferring far fewer parameters than would estimating the demand functions themselves.

Actually estimating the demand manifold can be done in different ways. One approach would be to assume a general functional form for the manifold. Such an estimated demand manifold is always numerically integrable, which allows us to infer some of the properties of the implied demand function. Implementing this approach in full would require a set of observations on pass-through and markups for a group of firms. We are not aware of any such data available to date. However, we can illustrate how the approach could be implemented by using the empirical results from De Loecker et al. (2016). They estimate the average pass-through from costs to prices for a sample of Indian firms, and also estimate the markups for each firm. Hence, we can estimate the demand parameters faced by the average firm without making any assumptions about the form of demand.

Alternatively, a more structural approach would be to assume a particular parametric family of demand functions, and to find the manifold from this family that best fits the data. Again, implementing this approach for most demand functions would require a set of observations on pass-through and markups for a group of firms which we do not have. However, for the CEPT demand function (introduced in Section II.E), which implies the same degree of pass-through but different markups for each firm, we can again illustrate this approach using the data from De Loecker et al. (2016).

In either case, estimating the manifold provides considerable information about the form of demand, and especially about its key implications for comparative statics, without the need to estimate the demand function directly. After briefly discussing the data, we illustrate each of these approaches in turn. 
De Loecker et al. (2016) give three estimates of average pass-through, one using ordinary least squares (OLS) and two using instrumental variables (IV). We use the OLS and the first of their IV estimates, for reasons discussed in Appendix B17. The OLS estimate of $k$ is 0.337 with a $95 \%$ confidence interval of 0.257 to 0.417 ; the IV estimate is 0.305 with a $95 \%$ confidence interval of 0.140 to 0.470 . These estimated confidence intervals for $k$ imply estimated confidence intervals for the convexity of demand faced by the average firm, using the expression for constant proportional pass-through from equation (14). These are illustrated by the horizontal boundaries of the regions in Figures 10 and 11: the darker region (in Figure 10) represents the confidence region implied by the OLS estimate of the pass-through coefficient, while the lighter region (in both figures) represents the confidence region implied by the IV estimate.

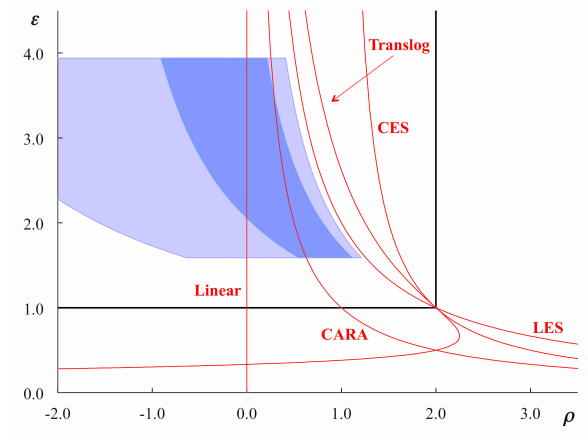

(a) Comparison with Demand Manifolds

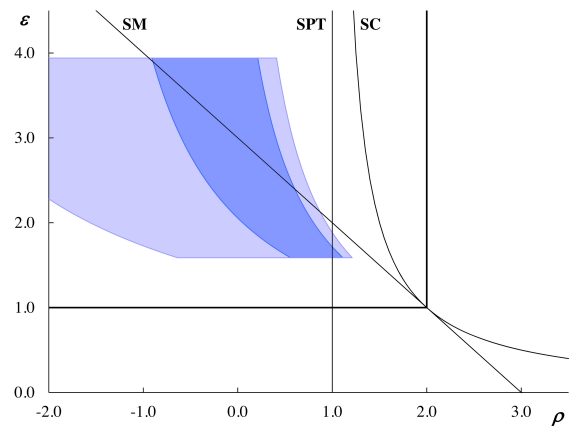

(b) Comparison with Comparative Statics

Figure 10. Range of Estimated Values of Elasticity and Convexity of the Average Firm

Note: Shaded areas are confidence regions for $\varepsilon$ and $\rho$ implied by data on $m$ and $k$ from De Loecker et al. (2016). (See text for details.)

To implement our first approach, we want to match these estimates of the convexity faced by the average firm with an estimate of the elasticity faced by the same firm. The published data from De Loecker et al. (2016) do not provide this, but do give both the mean and the median of the markup distribution. In the absence of any other information on the markup distribution, we take the range between the mean and the median as a plausible zone of central tendency for the markup of the firm with the average pass-through elasticity. We then use equation (40)(i) to back out estimates of the elasticity faced by this average firm. Combining these estimates with those of the average convexity allows us to construct two "confidence regions" in $(\varepsilon, \rho)$ space for the estimated elasticity and convexity faced by the average firm. Figure 10 illustrates. Panel (a) shows how the confidence regions compare with the demand manifolds implied by some of the most widely-used demand functions. Clearly, the data are consistent with both linear and CARA demands. By contrast, they are less consistent with LES 
and translog demands, and even less so with CES. Panel (b) shows how the confidence regions relate to the regions of comparative statics responses identified in Section I. Given that the results are not consistent with CES demands, it follows that superconvexity is ruled out, which supports the focus in a long literature stemming from Krugman (1979) that concentrates on the "plausible case" of subconvexity. By contrast, neither super- nor submodularity is ruled out: the data are consistent with large firms selecting into activities with either relatively low or relatively high marginal access costs. These conclusions suggest that combining our approach with more detailed estimates of pass-through and markups is likely to prove a fruitful direction for future research.

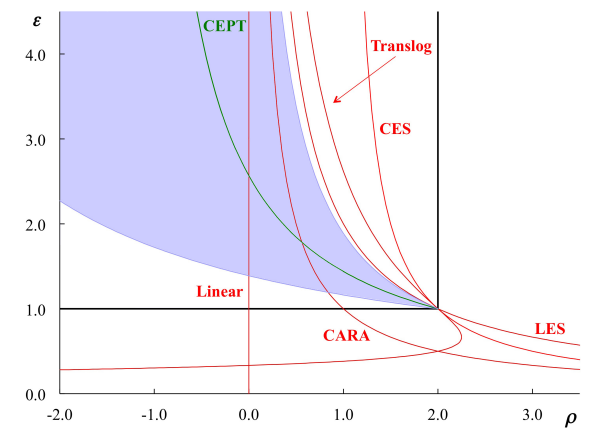

(a) Comparison with Demand Manifolds

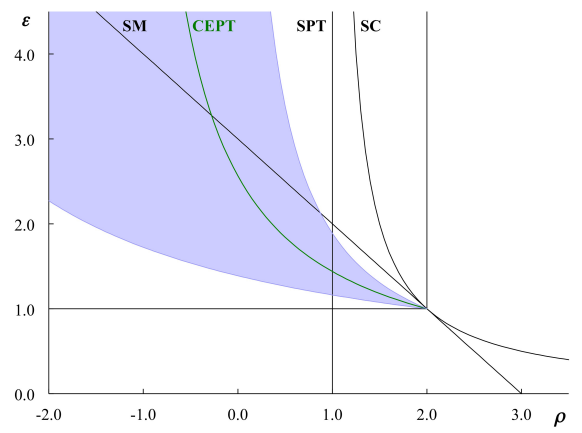

(b) Comparison with Comparative Statics

Figure 11. Calibrated Cept Demand Manifold

Note: Green curves represent the calibrated demand manifold from the CEPT family implied by IV estimates of $m$ and $k$ from De Loecker et al. (2016). Shaded areas give the implied 95\% confidence interval. (See text for details.)

To implement our second approach, we need to calibrate a particular member of the CEPT class of demand manifolds. This is easily done since it requires only an estimate of the pass-through elasticity $k$. (Recall Figure 2(a).) We choose to use the IV estimate of 0.305 : the resulting manifold is illustrated by the solid green curves labeled "CEPT" in Figure 11. Panel (a) shows how the estimated CEPT manifold compares with the manifolds for some standard demand functions. Panel (b) yields implications for comparative statics that are similar to those from Figure 10(b): superconvexity is clearly ruled out, while supermodularity holds for smaller firms but not for larger ones (those facing an elasticity of 3.28 or lower). A further feature of note is that the manifold implies less than onefor-one absolute pass-through for all but the very largest firms (those facing an elasticity of 1.44 or lower).

Needless to say, these exercises are illustrative only. Nevertheless, the results are very suggestive, and point to further potential applications of our approach, as data sets with more disaggregated information on pass-through for groups of 
firms or even individual firms become available. ${ }^{39}$

\section{B. Non-Parametric Counterfactuals: Income Elasticities}

Additional assumptions are needed to carry out counterfactual analysis. As in Section III, and following a large empirical literature, we assume that preferences are additively separable, though without any parametric restrictions on functional form. This guarantees both manifold invariance (from Corollary 3), and what Deaton (1974) calls "Pigou's Law": income and price elasticities are proportional to each other. ${ }^{40}$ In general the relationship is only approximately proportional: see Appendix B18 for details. However, the error term in the approximation depends on the budget share of the good, so, in the continuum case, the relationship is exactly proportional: ${ }^{41}$

$$
\eta_{i}=\Phi^{-1} \varepsilon_{i i}
$$

where $\eta_{i}$ and $\varepsilon_{i i}$ are the income and own-price elasticities of good $i$ respectively. The factor of proportionality $\Phi \equiv(d \log \lambda / d \log I)^{-1}$ is the inverse of the elasticity of the marginal utility of income with respect to income. Like $\lambda$ itself, $\Phi$ varies with income and prices; however, it is constant in a cross-section, so at a point in time it is the same for all goods. Hence, starting with an empirical manifold estimated from data on pass-through and markups as in Section IV.A, we can obtain estimates of the corresponding income elasticities, up to a factor of proportionality. ${ }^{42}$ These income elasticities can then be used to carry out counterfactual analyses of prices as done by Simonovska (2015). Note that we do not need to make any assumption about the distribution of firm productivities in this sub-section.

Additive separability is of course a restrictive assumption (as discussed by Deaton). However, in empirical applications with many thousands of goods, as is increasingly the norm in applied fields such as international trade, it is essential to impose some structure on the data. Allowing for non-homothetic tastes, even subject to Pigou's Law, seems at least as attractive as imposing homotheticity, as in the widely-used CES or homothetic translog specifications. Alternatively, if, as is likely, estimates of income elasticities are available from other sources, this relationship makes it possible in principle to assess the empirical validity of additively separable preferences.

\footnotetext{
${ }^{39}$ Estimating pass-through elasticities for individual firms will require panel data sets with sufficient time variation to avoid the need to pool data across firms.

${ }^{40}$ We discuss the case of directly additive preferences only. Similar results hold for indirect additivity, as shown by Deaton (1974).

${ }^{41}$ In empirical applications, it would be natural to allow for deviations from exact proportionality, though in practice they are likely to be very slight if the data are even moderately disaggregated. See equation (B47) in Appendix B18.

${ }^{42}$ The levels of the income elasticities in turn can be pinned down using extraneous information, or alternatively, if the goods in question exhaust the consumer's budget, using the Engel aggregation condition $\Sigma_{i} \omega_{i} \eta_{i}=1$, where $\omega_{i}$ is the budget share of good $i$.
} 


\section{Parametric Counterfactuals: Gains from Trade}

Further steps are possible if we are willing to make specific parametric assumptions about the form of additively separable preferences and about the distribution of firm productivities. These additional assumptions are the final building blocks needed for counterfactual analyses of welfare, drawing on the results in Section III.C. The first of these allows us to infer the elasticity of utility of a particular good from the elasticity and convexity of demand for that good. The second allows us to deduce the welfare effects of changes in consumption at the intensive and extensive margins. The demand manifold can be a sufficient statistic for the gains from trade only in the special case of homogeneous firms. However, it is an essential benchmark for, and in some cases a lower bound to, the gains from trade in more general models.

\section{Conclusion}

In this paper we present a new way of relating the structure of demand functions to the comparative statics properties of monopolistic and monopolistically competitive markets. We begin by illustrating, using a "firm's-eye view" of demand, how the elasticity and convexity of demand determine many comparative statics responses. We then show how the relationship between these two parameters, which we call the "demand manifold," provides a parsimonious representation of an arbitrary demand function, and a sufficient statistic for many comparative statics results. The manifold is particularly useful when it is unaffected by changes in exogenous variables, a property we call "manifold invariance." We characterize the conditions that the elasticity and convexity must exhibit for manifold invariance to hold; we introduce some new families of demand systems that exhibit manifold invariance; and we show that they nest many of the most widely used functions in applied theory. For example, our "bipower direct" family provides a natural way of nesting translog, CES, and linear demand functions. ${ }^{43}$

To illustrate the usefulness of our approach, we show that the demand manifold allows a parsimonious way of understanding how monopolistically competitive economies adjust to external shocks, as well as a framework for quantifying the effects of globalization. We also show some potential empirical applications of the manifold. The demand manifold can be estimated much more easily than the underlying demand function, using only data on pass-through and markups. With additional assumptions it can in turn be used to calculate income elasticities of demand and the gains from globalization.

Many extensions of our approach naturally suggest themselves. There are many other topics where functional form plays a key role in determining the implications of a given set of assumptions, and where our approach of focusing on the elasticity and convexity of a pivotal function yields important insights. For example, in

\footnotetext{
${ }^{43}$ Alternative ways of nesting translog and CES demands, though with considerably more complicated demand manifolds, appear in Novy (2013) and in Pollak, Sickles and Wales (1984).
} 
ongoing work we show that the same approach can be applied to the slope of the demand function, viewed as a function in itself, to derive results on variable passthrough and departures from Gibrat's Law. Further applications to choice under uncertainty and to oligopoly immediately come to mind. As for our application to the effects of globalization in monopolistic competition, the framework we have presented can be extended to allow for trade costs. ${ }^{44}$ Finally, the families of demand functions we have introduced provide a natural setting for estimating relatively flexible functional forms, and direct attention towards the parameters that matter for comparative statics predictions.

\section{REFERENCES}

Aguirre, Iñaki, Simon Cowan, and John Vickers. 2010. "Monopoly Price Discrimination and Demand Curvature." American Economic Review, 100(4): 1601-1615.

Antràs, Pol, and Elhanan Helpman. 2004. "Global Sourcing." Journal of Political Economy, 112(3): 552-580.

Arkolakis, Costas, Arnaud Costinot, and Andrés Rodríguez-Clare. 2012. "New Trade Models, Same Old Gains?" American Economic Review, 102(1): 94-130.

Arkolakis, Costas, Arnaud Costinot, David Donaldson, and Andrés Rodríguez-Clare. 2012. "The Elusive Pro-Competitive Effects of Trade." working paper, MIT, revised July 2014.

Atkin, David, and Dave Donaldson. 2012. "Who's Getting Globalized? The Size and Nature of Intranational Trade Costs." working paper, Yale University.

Badinger, Harald. 2007. "Has the EU's Single Market Programme Fostered Competition? Testing for a Decrease in Mark-Up Ratios in EU Industries." Oxford Bulletin of Economics and Statistics, 69(4): 497-519.

Baldwin, Richard, and James Harrigan. 2011. "Zeros, Quality, and Space: Trade Theory and Trade Evidence." American Economic Journal: Microeconomics, 3(2): 60-88.

Balistreri, Edward J., Russell H. Hillberry, and Thomas F. Rutherford. 2011. "Structural Estimation and Solution of International Trade Models with Heterogeneous Firms." Journal of International Economics, 83(2): 95-108.

Behrens, K., and Y. Murata. 2007. "General Equilibrium Models of Monopolistic Competition: A New Approach." Journal of Economic Theory, 136(1): 776-787.

\footnotetext{
${ }^{44}$ The implications of combining trade costs and general non-CES preferences have been considered by Bertoletti and Epifani (2014), Arkolakis et al. (2012), and Mrázová and Neary (2014).
} 
Benkovskis, Konstantins, and Julia Wörz. 2014. "What Drives the Market Share Changes? Price versus Non-Price Factors." European Central Bank Working Paper 1640.

Bertoletti, Paolo, and Federico Etro. 2016. "Preferences, Entry, and Market Structure." RAND Journal of Economics, 47(4): 792-821.

Bertoletti, Paolo, and Federico Etro. 2017. "Monopolistic Competition when Income Matters." Economic Journal (forthcoming).

Bertoletti, Paolo, and Paolo Epifani. 2014. "Monopolistic Competition: CES Redux?" Journal of International Economics, 93(2): 227-238.

Broda, Christian, and David E. Weinstein. 2006. "Globalization and the Gains from Variety." Quarterly Journal of Economics, 121(2): 541-585.

Bulow, Jeremy I., and Paul Pfleiderer. 1983. "A Note on the Effect of Cost Changes on Prices." Journal of Political Economy, 91(1): 182-185.

Bulow, Jeremy I., John D. Geanakoplos, and Paul D. Klemperer. 1985. "Multimarket Oligopoly: Strategic Substitutes and Complements." Journal of Political Economy, 93(3): 488-511.

Bustos, Paula. 2011. "Trade Liberalization, Exports and Technology Upgrading: Evidence on the Impact of MERCOSUR on Argentinian Firms." American Economic Review, 101(1): 304-340.

Chamberlin, Edward Hastings. 1933. The Theory of Monopolistic Competition: A Re-Orientation of the Theory of Value. Cambridge, Mass: Harvard University Press.

Deaton, Angus. 1974. "A Reconsideration of the Empirical Implications of Additive Preferences." Economic Journal, 84(334): 338-348.

Deaton, Angus, and John Muellbauer. 1980. "An Almost Ideal Demand System." American Economic Review, 70(3): 312-326.

De Loecker, Jan, Pinelopi K Goldberg, Amit K Khandelwal, and Nina Pavcnik. 2016. "Prices, Markups and Trade Reform." Econometrica, 84(2): 445-510.

Dhingra, Swati, and John Morrow. 2016. "Monopolistic Competition and Optimum Product Diversity under Firm Heterogeneity." mimeo, London School of Economics.

Diewert, W. Erwin. 1976. "Exact and Superlative Index Numbers." Journal of Econometrics, 4(2): 115-145.

Dixit, Avinash K., and Joseph E. Stiglitz. 1977. "Monopolistic Competition and Optimum Product Diversity." American Economic Review, 67(3): 297-308. 
Dixit, Avinash K., and Joseph E. Stiglitz. 1979. "Monopolistic Competition and Optimum Product Diversity: Reply [to Pettengill]." American Economic Review, 69(5): 961-963.

Dixit, Avinash K., and Joseph E. Stiglitz. 1993. "Monopolistic Competition and Optimum Product Diversity: Reply [to Yang and Heijdra]." American Economic Review, 83(1): 302-304.

Eeckhoudt, Louis, Christian Gollier, and Thierry Schneider. 1995. "RiskAversion, Prudence and Temperance: A Unified Approach." Economics Letters, 48(3): 331-336.

Fabinger, Michal, and E. Glen Weyl. 2012. "Pass-Through and Demand Forms." working paper, University of Chicago.

Feenstra, Robert C. 2003. "A Homothetic Utility Function for Monopolistic Competition Models, without Constant Price Elasticity." Economics Letters, 78(1): 79-86.

Feenstra, Robert C. 2014. "Restoring the Product Variety and ProCompetitive Gains from Trade with Heterogeneous Firms and Bounded Productivity." NBER Working Paper No. 16796.

Goldman, S. M., and H. Uzawa. 1964. "A Note on Separability in Demand Analysis." Econometrica, 32(3): 387-398.

Gopinath, Gita, and Oleg Itskhoki. 2010. "Frequency of price adjustment and pass-through." Quarterly Journal of Economics, 125(2): 675-727.

Helpman, Elhanan, Marc J. Melitz, and Stephen R. Yeaple. 2004. "Export Versus FDI with Heterogeneous Firms." American Economic Review, 94(1): 300-316.

Kimball, Miles S. 1992. "Precautionary Motives for Holding Assets." in P. Newman, M. Milgate and J. Eatwell (eds.): The New Palgrave Dictionary of Money and Finance, New York: Stockton Press.

Kimball, Miles S. 1995. "The Quantitative Analytics of the Basic Neomonetarist Model." Journal of Money, Credit and Banking, 27(4): 1241-1277.

Klenow, Peter J., and Jonathan L. Willis. 2016. "Real Rigidities and Nominal Price Changes." Economica, 83(331): 443-472.

Krugman, Paul R. 1979. "Increasing Returns, Monopolistic Competition, and International Trade." Journal of International Economics, 9(4): 469-479.

Marshall, Alfred. 1920. Principles of Economics, An Introductory Volume. . Eighth ed., London: Macmillan. 
Melitz, Marc J. 2003. "The Impact of Trade on Intra-Industry Reallocations and Aggregate Industry Productivity." Econometrica, 71(6): 1695-1725.

Melitz, Marc J., and Gianmarco I.M. Ottaviano. 2008. "Market Size, Trade, and Productivity." Review of Economic Studies, 75(1): 295-316.

Melitz, Marc J., and Stephen J. Redding. 2015. "New Trade Models, New Welfare Implications." American Economic Review, 105(3): 1105-46.

Mrázová, Monika, and J. Peter Neary. 2013. "Not so Demanding: Preference Structure, Firm Behavior, and Welfare." University of Oxford Economics Discussion Paper No. 691.

Mrázová, Monika, and J. Peter Neary. 2014. "Together at Last: Trade Costs, Demand Structure, and Welfare." American Economic Review, Papers and Proceedings, 104(5): 298-303.

Mrázová, Monika, and J. Peter Neary. 2018. "Selection Effects with Heterogeneous Firms." forthcoming in Journal of the European Economic Association.

Mrázová, Monika, J. Peter Neary, and Mathieu Parenti. 2015. "Sales and Markup Dispersion: Theory and Empirics." Department of Economics, University of Oxford Discussion Paper No. 774.

Muellbauer, John. 1975. "Aggregation, Income Distribution and Consumer Demand." Review of Economic Studies, 42(4): 525-543.

Neary, J. Peter. 2009. "Putting the 'New' into New Trade Theory: Paul Krugman's Nobel Memorial Prize in Economics." Scandinavian Journal of Economics, 111(2): 217-250.

Novy, Dennis. 2013. "International Trade without CES: Estimating Translog Gravity." Journal of International Economics, 89(2): 271-282.

Parenti, Mathieu, Philip Ushchev, and Jacques-François Thisse. 2017. "Toward a Theory of Monopolistic Ccompetition." Journal of Economic Theory, 167: 86-115.

Pettengill, John S. 1979. "Monopolistic Competition and Optimum Product Diversity: Comment." American Economic Review, 69(5): 957-960.

Pollak, Robert A. 1971. "Additive Utility Functions and Linear Engel Curves." Review of Economic Studies, 38(4): 401-414.

Pollak, Robert A. 1972. "Generalized Separability." Econometrica, 40(3): pp. 431-453.

Pollak, Robert A., Robin C. Sickles, and Terence J. Wales. 1984. "The CES-Translog: Specification and Estimation of a New Cost Function." Review of Economics and Statistics, 66(4): 602-607. 
Schmalensee, Richard. 1981. "Output and Welfare Implications of Monopolistic Third-Degree Price Discrimination." American Economic Review, 71(1): 242-247.

Seade, Jesus. 1980. "On the Effects of Entry." Econometrica, 48(2): 479-489.

Shapiro, Carl. 1989. "Theories of Oligopoly Behavior." in R. Schmalensee and R. Willig (eds.): Handbook of Industrial Organization, Elsevier, 329-414.

Simonovska, Ina. 2015. "Income Differences and Prices of Tradables: Insights from an Online Retailer." Review of Economic Studies, 82(4): 1612-1656.

Soderbery, Anson. 2015. "Estimating Import Supply and Demand Elasticities: Analysis and Implications." Journal of International Economics, 96(1): 1-17.

Spearot, Alan C. 2012. "Firm Heterogeneity, New Investment and Acquisitions." Journal of Industrial Economics, 60(1): 1-45.

Spearot, Alan C. 2013. "Market Access, Investment, and Heterogeneous Firms." International Economic Review, 54(2): 601-627.

Vives, Xavier. 1999. Oligopoly Pricing: Old Ideas and New Tools. Cambridge, Mass.: MIT Press.

Ward, Michael B, Jay P Shimshack, Jeffrey M Perloff, and J Michael Harris. 2002. "Effects of the Private-Label Invasion in Food Industries." American Journal of Agricultural Economics, 84(4): 961-973.

Weyl, E. Glen, and Michal Fabinger. 2013. "Pass-Through as an Economic Tool: Principles of Incidence under Imperfect Competition." Journal of Political Economy, 121(3): 528-583.

Zhelobodko, Evgeny, Sergey Kokovin, Mathieu Parenti, and JacquesFrançois Thisse. 2012. "Monopolistic Competition: Beyond the Constant Elasticity of Substitution." Econometrica, 80(6): 2765-2784.

\section{APPENDIX}

\section{A1. Alternative Measures of Slope and Curvature}

As our measure of demand slope, we follow standard practice and work throughout with the price elasticity of demand, which can be expressed in terms of the derivatives of either the inverse or the direct demand functions $p(x)$ and $x(p)$ : $\varepsilon \equiv-p / x p^{\prime}=-p x^{\prime} / x$. An alternative would be to use instead the inverse of this elasticity, $e \equiv-x / p x^{\prime}=1 / \varepsilon{ }^{45}$ This has the advantage that, since $e=-x p^{\prime} / p$,

\footnotetext{
${ }^{45}$ It equals the elasticity of marginal utility: $e=-d \log u^{\prime}(x) / d \log x$; with additive separability, it has been called the "relative love for variety" by Zhelobodko et al. (2012); and, in monopoly equilibrium, it equals the profit margin or Lerner Index of monopoly power: $e=(p-c) / p$.
} 
its definition is symmetric with those of demand curvature $\rho \equiv-x p^{\prime \prime} / p^{\prime}$, and of $\chi \equiv-x p^{\prime \prime \prime} / p^{\prime \prime}$, a unit-free measure of the third derivative of the demand function, which, following Kimball (1992) and Eeckhoudt, Gollier and Schneider (1995), we call the "Coefficient of Relative Temperance," or simply "temperance". (This parameter proves useful in some of the proofs and derivations in the online appendix.) Offsetting advantages of using $\varepsilon$ include its greater intuitive appeal, and the fact that it focuses attention on the region of parameter space where comparative statics results are ambiguous.

Turning to measures of curvature, the convexity of inverse demand which we use throughout equals the elasticity of the slope of inverse demand, $\rho \equiv$ $-x p^{\prime \prime} / p^{\prime}=-d \log p^{\prime}(x) / d \log x$. Its importance for comparative statics results was highlighted by Seade (1980), and it is widely used in industrial organization, for example by Bulow, Geanakoplos and Klemperer (1985) and Shapiro (1989). An alternative measure is the convexity of the direct demand function $x(p): r(p) \equiv-p x^{\prime \prime}(p) / x^{\prime}(p)$. A convenient property is that $e$ and $r$ are dual to $\varepsilon$ and $\rho$ :

$$
\begin{array}{ll}
e \equiv-\frac{x}{p x^{\prime}}=\frac{1}{\varepsilon} & r \equiv-\frac{p x^{\prime \prime}}{x^{\prime}}=\frac{p p^{\prime \prime}}{\left(p^{\prime}\right)^{2}}=\varepsilon \rho \\
\varepsilon \equiv-\frac{p}{x p^{\prime}}=\frac{1}{e} & \rho \equiv-\frac{x p^{\prime \prime}}{p^{\prime}}=\frac{x x^{\prime \prime}}{\left(x^{\prime}\right)^{2}}=e r
\end{array}
$$

We use these properties in the proof of Proposition 3 in Appendix B6 below. Yet another measure of demand curvature, widely used in macroeconomics, is the superelasticity of Kimball (1995), defined as the elasticity with respect to price of the elasticity of demand, $S \equiv d \log \varepsilon / d \log p$. Positive values of $S$ allow for asymmetric price setting in monopolistic competition. It is related to our measures as follows: $S=(d \log \varepsilon / d \log x)(d \log x / d \log p)=\left(x \varepsilon_{x} / \varepsilon\right)(-\varepsilon)=\varepsilon+$ $1-\varepsilon \rho$ (the last step using Appendix B1), so it is positive if and only if demand is subconvex. Figure A1(a) illustrates loci of constant superelasticity, $\rho=(\varepsilon+$ $1-S) / \varepsilon$. Formally, they correspond to the family of Pollak manifolds, $\bar{\rho}(\varepsilon)=$ $(\sigma+1) / \varepsilon$, displaced rightwards to be symmetric around the log-linear $(\rho=1)$ rather than the linear $(\rho=0)$ demand manifold.

Kimball himself did not present a parametric family of demand functions. Klenow and Willis (2016) introduce a parametric family which has the property that the superelasticity is a linear function of the elasticity: $S=b \varepsilon$. Substituting for $S$ leads to the family of demand manifolds $\bar{\rho}(\varepsilon)=((1-b) \varepsilon+1) / \varepsilon$, which are lateral displacements of the CES locus. Figure A1(b) illustrates some members of this family.

We note in footnotes some implications of these alternative measures. The choice between them is largely a matter of convenience. We express all our results in terms of $\varepsilon$ and $\rho$, partly because this is standard in industrial organization, 


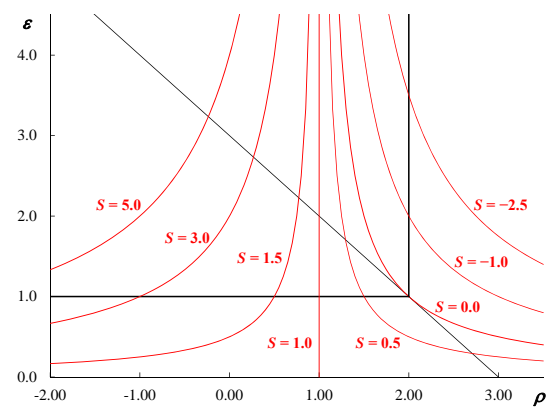

(a) Iso-Superelasticity Loci

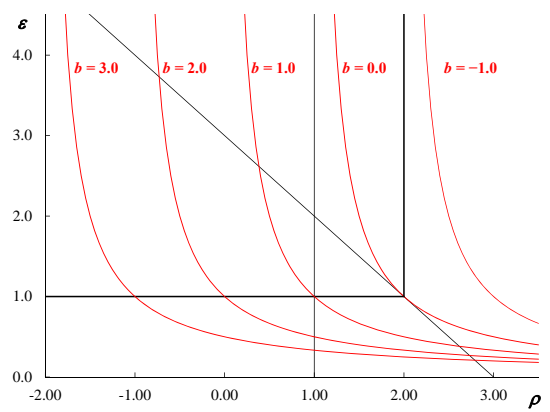

(b) Klenow-Willis Demand Manifolds

Figure A1. Kimball Superelasticity

partly because (unlike $e$ and $r$ ) the inverse demand functions are easily integrated to obtain the direct utility function, and partly because (unlike $\varepsilon$ and $S$ ) they lead to simple restrictions on the shape of the demand manifold as shown in Proposition 3. However, our results could also be expressed in terms of $e$ and $r$ or of $\varepsilon$ and $S$.

\section{A2. Oligopoly}

We consider only monopoly and monopolistic competition in the text, but our approach can also be applied to oligopolistic markets. Even in the simplest case of Cournot competition between $n$ firms producing an identical good, this leads to extra complications. Now we need to distinguish market demand $X$ from the sales of an individual firm $i, x_{i}$, with the elasticity and convexity of the demand function $p(X)$ defined in terms of the former: $\varepsilon \equiv-p / X p^{\prime}$ and $\rho \equiv-X p^{\prime \prime} / p^{\prime}$. The first-order condition is now $p+x_{i} p^{\prime}=c_{i} \geq 0$, while the second-order condition is $2 p^{\prime}+x_{i} p^{\prime \prime}<0$. The implied restrictions on the size of the admissible region can be expressed in terms of market shares $\left(\omega_{i} \equiv x_{i} / X\right)$. The first-order condition implies that $\varepsilon \geq \max _{i}\left(\omega_{i}\right)$, which attains its lower bound of $1 / n$ when firms are identical. As for the second-order condition, it implies that $\rho<2 \min _{i}\left(1 / \omega_{i}\right)$, which attains its upper bound of $2 n$ when firms are identical. Since (except when firms are identical) market shares are endogenous, the same is true of the size of the admissible region. A different restriction on convexity comes from the stability condition: $\rho<n+1$. This imposes a tighter bound than the secondorder condition provided the largest firm is not "too" large: $\max _{i}\left(\omega_{i}\right)<2 /(n+1)$. Relative to the monopoly case, the admissible region expands unambiguously, except in the boundary case of a dominant firm, where $\max _{i}\left(\omega_{i}\right)=1$.

Equally important in oligopoly, as we know from Bulow, Geanakoplos and Klemperer (1985), is that many comparative statics results hinge on strategic substitutability: the marginal revenue of firm $i$ is decreasing in the output of 
every other firm. This is equivalent to $p^{\prime}+x_{i} p^{\prime \prime}<0, \forall i$, which in our notation implies a restriction on convexity that lies within the admissible region: $\rho<\min _{i}\left(1 / \omega_{i}\right) \geq 1$, which attains its upper bound of $n$ when firms are identical.

\section{A3. Examples Illustrating Proposition 2: Manifold Invariance}

Sections II.C and II.D give details of demand functions that satisfy the conditions of Proposition 2 for manifold invariance to hold. Here we introduce two very different classes of demand function that also exhibit manifold invariance. ${ }^{46}$

The first of these is the CEPT or Constant Elasticity of Pass-Through family, discussed in Section II.E. The implied expressions for the elasticity and convexity as functions of output $x$, are:

$$
\varepsilon(x)=\frac{x^{\frac{k-1}{k}}+\gamma}{\gamma} \quad \rho(x)=2-\frac{1}{k} \frac{x^{\frac{k-1}{k}}}{x^{\frac{k-1}{k}}+\gamma}
$$

The requirements that prices be positive, that $\varepsilon$ be greater than one, and that $\rho$ be less than two, imply that all three coefficients, $\beta, \gamma$, and $k$, must be positive. The coefficient $k$ also determines whether the demand function is sub- or superconvex: CEPT demands are subconvex, with $\varepsilon_{x} \leq 0$, if and only if $k$ is less than one. It follows immediately from (A3) that $\varepsilon(x)$ and $\rho(x)$ satisfy condition (11a) from Proposition 2 for manifold invariance with respect to $\gamma$ : they depend on $\gamma$ only through a common factor $f=F(x, \gamma) \equiv \gamma^{-1} x^{(k-1) / k}$. Thus $\varepsilon=f+1$ and $\rho=2-(f+1) / f k$; combining recovers the manifold as in (6). Note that, although all firms have the same elasticity of pass-through, this demand function allows for variable markups. Writing the markup as $m \equiv p / c=\varepsilon /(\varepsilon-1)$ :

$$
m(x)=\frac{\gamma}{x^{\frac{k-1}{k}}}
$$

Naturally, markups are increasing in firm size, $m_{x} \geq 0$, if and only if demands are subconvex, $k \leq 1$.

The second class of demand function that exhibits manifold invariance is a generalization of the "CREMR" demand function introduced in Mrázová, Neary and Parenti (2015):

$$
p(x)=\beta x^{-\eta}(x-\gamma)^{-\theta}
$$

When $\eta=1$, this reduces to the CREMR case, so-called because it exhibits a constant revenue elasticity of marginal revenue. For the demand function in (A5)

\footnotetext{
${ }^{46}$ If we assume additive separability, both of these demand functions imply sub-utility functions that take a hypergeometric form. Though analytically complex, this is straightforward to program, so these functions could be used as a basis for quantitative studies of welfare questions.
} 
the elasticity and convexity can be written as:

$$
\varepsilon=(\eta+\theta f)^{-1} \quad \text { and } \quad \rho=\eta+\theta f+\frac{\eta+\theta f^{2}}{\eta+\theta f}
$$

where $f=F(x, \gamma) \equiv x /(x-\gamma)$. These clearly satisfy condition (11a) from Proposition 2: both elasticity and convexity depend on $x$ and $\gamma$ through a common function $F(x, \gamma)$. Hence the demand manifold is invariant with respect to the parameter $\gamma$ :

$$
\bar{\rho}(\varepsilon)=\frac{1}{\theta \varepsilon}\left[(\eta+\theta) \eta \varepsilon^{2}-2 \eta \varepsilon+\theta+1\right]
$$

\section{A4. Heterogeneous Firms with Additive Separability}

The first step is to calculate the elasticities of the maximum operating profit function:

$$
\pi(c, \lambda, k) \equiv \max _{y}[p(y, \lambda, k)-c] y \quad \text { where } \quad p(y, \lambda, k)=\lambda^{-1} u^{\prime}(y / k L)
$$

For later use, the first and second derivatives of the inverse demand function, expressed in terms of elasticities, are:

$$
\begin{aligned}
& \frac{y p_{y}}{p}=-\frac{1}{\varepsilon}, \quad \frac{\lambda p_{\lambda}}{p}=-1 \quad \text { and } \quad \frac{k p_{k}}{p}=\frac{1}{\varepsilon} \\
& \frac{y p_{y y}}{p_{y}}=-\rho, \quad \frac{y p_{y \lambda}}{p_{\lambda}}=-\frac{1}{\varepsilon} \quad \text { and } \quad \frac{y p_{y k}}{p_{k}}=\frac{1}{\varepsilon}
\end{aligned}
$$

Using the envelope theorem, the derivatives of the profit function are:

$$
\pi_{c}=-y, \quad \pi_{\lambda}=-\lambda^{-2} u^{\prime} y=-\lambda^{-1} p y, \quad \pi_{k}=-\frac{y^{2} u^{\prime \prime}}{\lambda k^{2} L}=-\frac{y^{2}}{k} p_{y}
$$

These in turn can be expressed in terms of elasticities, making use of the firstorder condition $p+y p_{y}=c$ :

$$
\begin{gathered}
\frac{c \pi_{c}}{\pi}=-\frac{c y}{\pi}=-\frac{c}{p-c}=-(\varepsilon-1), \quad \frac{\lambda \pi_{\lambda}}{\pi}=-\frac{p y}{\pi}=-\frac{p}{p-c}=-\varepsilon, \\
\frac{k \pi_{k}}{\pi}=-\frac{y^{2} p_{y}}{(p-c) y}=\frac{y p_{y}}{y p_{y}}=1
\end{gathered}
$$


Aggregating these gives the elasticities of aggregate profits:

$$
\begin{gathered}
\frac{\lambda \bar{v}_{\lambda}}{\bar{v}}=\int_{\underline{c}}^{\bar{c}} \frac{v(c, \lambda, k)}{\bar{v}(\lambda, k)} \frac{\lambda \pi_{\lambda}(c)}{\pi(c)} g(c) \mathrm{d} c=-\int_{\underline{c}}^{\bar{c}} \frac{v(c, \lambda, k)}{\bar{v}(\lambda, k)} \varepsilon(c) g(c) \mathrm{d} c=-\bar{\varepsilon} \\
\frac{k \bar{v}_{k}}{\bar{v}}=\int_{\underline{c}}^{\bar{c}} \frac{v(c, \lambda, k)}{\bar{v}(\lambda, k)} \frac{k \pi_{k}(c)}{\pi(c)} g(c) \mathrm{d} c=\int_{\underline{c}}^{\bar{c}} \frac{v(c, \lambda, k)}{\bar{v}(\lambda, k)} g(c) \mathrm{d} c=1
\end{gathered}
$$

where the final step makes use of the definition of $\bar{v}(\lambda, k)$ from (28). Note that these aggregate effects are a weighted average of the elasticities of operating profits, weighted by each firm's share in expected total profits: naturally, the weights attached to firms that choose not to enter and so earn zero profits are themselves zero.

Using these results, we can solve for the effect of globalization on the degree of competition $\lambda$ by totally differentiating the zero-expected-profit condition (28):

$$
\hat{\lambda}=-\left(\frac{\lambda \bar{v}_{\lambda}}{\bar{v}}\right)^{-1} \frac{k \bar{v}_{k}}{\bar{v}}=\frac{1}{\bar{\varepsilon}} \hat{k}
$$

The next step is to solve for the effects of globalization at the intensive margin. Totally differentiating the first-order condition, and making use of (A9) and (A10), the partial elasticities of output with respect to $\lambda$ and $k$ are given by:

$$
\frac{\lambda y_{\lambda}}{y}=-\frac{\varepsilon-1}{2-\rho} \quad \text { and } \quad \frac{k y_{k}}{y}=1
$$

Hence, the total derivative of output with respect to $k$, allowing for the indirect effect via the level of competition, is:

$$
\hat{y}=\left(\frac{k y_{k}}{y}+\frac{\lambda y_{\lambda}}{y} \frac{k}{\lambda} \frac{d \lambda}{d k}\right) \hat{k}=\left(1-\frac{\varepsilon-1}{2-\rho} \frac{1}{\bar{\varepsilon}}\right) \hat{k}
$$

where we use (A16) and (A17) to simplify. Rearranging gives the decomposition in (31) in the text.

As for prices, equation (20) in Section III.A, which relates price changes to changes in per capita consumption $x$, continues to hold for each individual firm. The change in $x$ in turn can be derived from the goods-market equilibrium condition (22):

$$
\hat{x}=\hat{y}-\hat{k}=\left(1-\frac{\varepsilon-1}{2-\rho} \frac{1}{\bar{\varepsilon}}-1\right) \hat{k}=-\frac{\varepsilon-1}{2-\rho} \frac{1}{\bar{\varepsilon}} \hat{k}
$$

Substituting into (20) and rearranging gives the change in prices in (32). 


\title{
Not so Demanding: Demand Structure and Firm Behavior
}

\author{
By Monika Mrázová and J. Peter Neary
}

Supplementary Online Appendix B

\section{B1. Preliminaries: A Key Lemma}

We make repeated use of the following result:

LEMMA 4: Consider a twice-differentiable function $g(x)$. Both the double-log convexity of $g(x)$ and the rate of change of its elasticity can be expressed in terms of its first and second derivatives as follows:

$$
\frac{d^{2} \log g}{d(\log x)^{2}}=x \frac{d}{d x}\left(\frac{x g^{\prime}}{g}\right)=\frac{x g^{\prime}}{g}\left(1-\frac{x g^{\prime}}{g}+\frac{x g^{\prime \prime}}{g^{\prime}}\right)
$$

For most of the paper, $g(x)$ is the inverse demand function $p(x)$, and (B1) can be expressed in terms of the demand elasticity and convexity:

$$
\frac{d^{2} \log p}{d(\log x)^{2}}=\frac{x \varepsilon_{x}}{\varepsilon^{2}}=-\frac{1}{\varepsilon}\left(1+\frac{1}{\varepsilon}-\rho\right)
$$

Recalling equation (4), this gives the result in Section I.B that the elasticity of demand increases with sales if and only demand is superconvex. Qualitatively the same outcome comes from applying Lemma 4 to the direct demand function, replacing $g(x)$ by $x(p)$, and making use of (A1) and (A2):

$$
\frac{d^{2} \log x}{d(\log p)^{2}}=-p \frac{d \varepsilon}{d p}=-\varepsilon(1+\varepsilon-\varepsilon \rho)
$$

We use a different application of the Lemma to prove Proposition 1 in Appendix B2 below. Now, let $g(x)$ denote the absolute value of the demand slope $-p^{\prime}(x)$, so (B1) becomes:

$$
\frac{d^{2} \log \left(-p^{\prime}\right)}{d(\log x)^{2}}=-x \rho_{x}=-\rho(1+\rho-\chi)
$$

where $\chi$ is the temperance parameter, defined in Appendix A1. The result in (B4) that the change in convexity as sales rise depends only on temperance and convexity itself parallels that in (B2) that the change in elasticity as sales rise depends only on convexity and elasticity itself.

All these expressions are zero in the CES case given by (4), when all three parameters depend only on the elasticity $\sigma:\{\varepsilon, \rho, \chi\}_{C E S}=\left\{\sigma, 1+\frac{1}{\sigma}, 2+\frac{1}{\sigma}\right\}$. 


\section{B2. Proof of Proposition 1: Existence of the Manifold}

We wish to prove that, except in the CES case, only one of $\varepsilon_{x}$ and $\rho_{x}$ can be zero at any $x$. Recall from equations (B2) and (B4) that $\varepsilon_{x}=\frac{\varepsilon}{x}\left(\rho-\frac{\varepsilon+1}{\varepsilon}\right)$ and $\rho_{x}=\frac{\rho}{x}(1+\rho-\chi)$, where $\chi \equiv-\frac{x p^{\prime \prime \prime}}{p^{\prime \prime}}$. We have already seen that $\varepsilon_{x}$ can be zero only along the CES locus. As for $\rho_{x}=0$, there are two cases where it can equal zero. The first is where $\rho=0$. From (B4), this implies that $\varepsilon_{x}$ equals $-\frac{\varepsilon+1}{x}$, which is non-zero. The second is where $1+\rho-\chi=0$. As we saw in Section II.D, this implies that the demand function takes the iso-convex or Bulow-Pfleiderer form: $p(x)=\alpha+\beta x^{-\theta}$. The intersection of this with $\varepsilon_{x}=0$ is the CES limiting case of Bulow-Pfleiderer as sales tend towards zero: see Figure B3(a) below. Hence we can conclude that the only cases where both $\varepsilon_{x}$ and $\rho_{x}$ equal zero at a given $x$ lie on a CES demand function.

\section{B3. Proof of Proposition 2: Manifold Invariance}

We wish to prove that one or other of the conditions in Proposition 2 is necessary and sufficient for a given demand manifold to be invariant with respect to a demand parameter $\phi$. Of the two conditions, one relates to properties of inverse demand functions and the other to those of direct demand functions. However, it is clear by inspection that the two conditions are equivalent to each other except that $\varepsilon$ and $\rho$ are functions of $x$ in one case but of $p$ in the other. Hence we need only prove the conditions in one case. In what follows, we consider only the case where $\varepsilon$ and $\rho$ are derived from the inverse demand function and so depend on $x$ and $\phi$. The restriction we derive is thus necessary and sufficient for manifold invariance, conditional on the elasticity and convexity being derived from the inverse demand function.

Recall that, when $\rho_{x}$ is non-zero, which implies that $\varepsilon_{x}$ is also non-zero, the demand manifold can be locally written either as $\varepsilon=\bar{\varepsilon}(\rho, \phi)$ or as $\rho=\bar{\rho}(\varepsilon, \phi)$ where $\phi$ is a vector parameter. ${ }^{47}$

Let us first prove sufficiency. Suppose $\varepsilon$ and $\rho$ depend on $x$ and $\phi$ through a common sub-function of $x$ and $\phi$ as stated in the proposition: $\varepsilon(x, \phi)=\tilde{\varepsilon}[F(x, \phi)]$ and $\rho(x, \phi)=\tilde{\rho}[F(x, \phi)]$. Eliminating $F$ from this system yields a relation between $\varepsilon$ and $\rho$, which is independent of $\phi$.

Now let us prove necessity. Without loss of generality suppose that the demand manifold can be locally written as:

$$
\varepsilon=\bar{\varepsilon}(\rho, \phi)
$$

Let us define $F$ by $F(x, \phi)=\rho(x, \phi)$. From (B5), we have $\varepsilon(x, \phi)=\bar{\varepsilon}[\rho(x, \phi), \phi]$.

\footnotetext{
${ }^{47}$ As explained in the text, we exclude from the proposition all Bulow-Pfleiderer demands, for which $\rho_{x}$ is zero, and in particular the CES special case, for which $\varepsilon_{x}$ is also zero. This also excludes the case where $\rho_{p}$ is zero. Since $\rho(p)=\frac{x(p) x^{\prime \prime}(p)}{\left(x^{\prime}(p)\right)^{2}}$ from (A2), the only demand function with $\rho_{p}=0$ is the linear, which is a special case of the Bulow-Pfleiderer class.
} 
Since the demand manifold is independent of $\phi$ by assumption, we have $\bar{\varepsilon}(\rho, \phi)=$ $\bar{\varepsilon}(\rho)$ and so $\varepsilon(x, \phi)=\bar{\varepsilon}[\rho(x, \phi)]=\bar{\varepsilon}[F(x, \phi)]$. Hence both $\varepsilon$ and $\rho$ depend on $x$ and $\phi$ only through the common sub-function $F$.

\section{B4. Proof of Corollary 2: Multiplicative Separability}

If demands are multiplicatively separable in $\phi$, both the elasticity and convexity are independent of $\phi$. In the case of inverse demands, $p(x, \phi)=\beta(\phi) \tilde{p}(x)$ implies:

$$
\varepsilon=-\frac{p(x, \phi)}{x p_{x}(x, \phi)}=-\frac{\tilde{p}(x)}{x \tilde{p}^{\prime}(x)} \quad \text { and } \quad \rho=-\frac{x p_{x x}(x, \phi)}{p_{x}(x, \phi)}=-\frac{x \tilde{p}^{\prime \prime}(x)}{\tilde{p}^{\prime}(x)}
$$

A special case of this is additive preferences: $\int_{\omega \in \Omega} u[x(\omega)] d \omega$. The first-order condition is $u^{\prime}[x(\omega)]=\lambda^{-1} p(\omega)$, which implies that the perceived indirect demand function can be written in multiplicative form: $p(x, \phi)=\lambda(\phi) \tilde{p}(x)$.

Similar derivations hold for direct demands. If $x(p, \phi)=\delta(\phi) \tilde{x}(p)$ then:

$$
\varepsilon=-\frac{p x_{p}(p, \phi)}{x(p, \phi)}=-\frac{p \tilde{x}^{\prime}(p)}{\tilde{x}(p)} \quad \text { and } \quad \rho=\frac{x(p, \phi) x_{p p}(p, \phi)}{\left[x_{p}(p, \phi)\right]^{2}}=\frac{\tilde{x}(p) \tilde{x}^{\prime \prime}(p)}{\left[\tilde{x}^{\prime}(p)\right]^{2}}
$$

We also have a similar corollary, the case of indirect additivity, where the indirect utility function can be written as: $\int_{\omega \in \Omega} v[p(\omega) / I] d \omega$. Roy's Identity implies that: $v^{\prime}[p(\omega) / I]=-\lambda x(\omega)$, where $\lambda$ is the marginal utility of income, from which the direct demand function facing a firm can be written in multiplicative form: $x(p / I, \phi)=-\lambda^{-1}(\phi) \tilde{x}(p / I)$.

\section{B5. Market Size and the Logistic Demand Function}

To illustrate Corollary 4 that the demand manifold is independent of market size, consider the logistic direct demand function, equivalent to a logit inverse demand function (see Cowan (2012)):

$$
x(p, s)=\left(1+e^{p-a}\right)^{-1} s \quad \Leftrightarrow \quad p(x, s)=a-\log \frac{x}{s-x}
$$

Here $x / s$ is the share of the market served: $x \in[0, s]$; and $a$ is the price that induces a $50 \%$ market share: $p=a$ implies $x=\frac{s}{2}$. The elasticity equals $\varepsilon=p \frac{s-x}{s}$, while the convexity equals $\rho=\frac{s-2 x}{s-x}$, which must be less than one. Eliminating $x$ and $p$ yields a closed-form expression for the manifold:

$$
\bar{\varepsilon}(\rho)=\frac{a-\log (1-\rho)}{2-\rho}
$$


which is invariant with respect to market size $s$ though not with respect to $a$. Figure B1 illustrates this for values of $a$ equal to 2 and $5 .{ }^{48}$

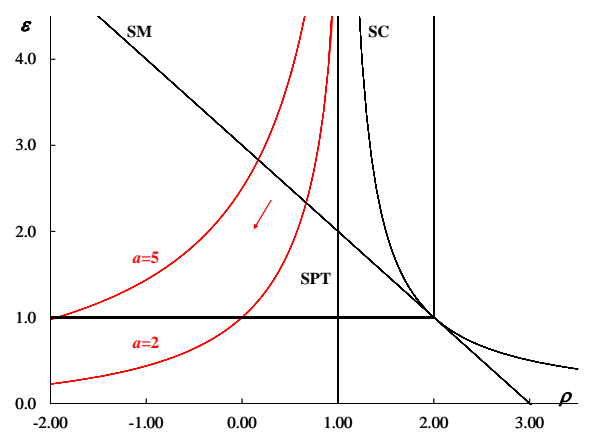

Figure B1. The Demand Manifold for the Logistic Demand Function

The logistic is just one example of a whole family of demand functions, many of which can be derived from log-concave distribution functions: Bergstrom and Bagnoli (2005) give a comprehensive review of these. The power of the approach introduced in the last section is that we can immediately state the properties of all these functions: they imply sub-pass-through and, a fortiori, subconvexity, while they are typically supermodular for low values of output and submodular for high values. Any shock, such as a partial-equilibrium increase in market size, that raises the output of a monopoly firm, implies an adjustment as shown by the arrow in the figure.

\section{B6. Proof of Proposition 3: Bipower Demands}

The inverse and direct bipower demand functions in (13a) and (13b) have very different implications for behavior. However, they have the same functional form except that the roles of $x$ and $p$ are reversed, so results proved for one can be applied immediately to the other. It is most convenient to focus on the inverse demands in (13a). Sufficiency follows by differentiating $p(x)$ and calculating the manifold directly. As in Section A3, we write the elasticity and convexity in a way which shows that they satisfy the conditions for manifold invariance in condition (11a) from Proposition 2. Necessity follows by setting $\rho(x)=a+b \varepsilon(x)$, where $a$ and $b$ are constants, and solving the resulting Euler-Cauchy differential equation. This proves the result in (13a): a bipower inverse demand function is necessary and sufficient for a manifold such that $\rho$ is affine in $\varepsilon$. With appropriate

\footnotetext{
${ }^{48}$ The value of $\rho$ determines market share and the level of price relative to $a: x=\frac{1-\rho}{2-\rho} s$ and $p=$ $a-\log (1-\rho)$. In particular, when the function switches from convex to concave (i.e., $\rho$ is zero), the elasticity equals $\frac{a}{2}$, market share is $50 \%$, and $p=a$.
} 
relabeling this in turn implies that a bipower direct demand function is necessary and sufficient for an affine dual manifold, that is to say, an equation linking the dual parameters $r$ and $e: \bar{r}(e)=\nu+\sigma+1-\nu \sigma e$. Recalling from (A1) that $e=\frac{1}{\varepsilon}$ and $r=\varepsilon \rho$ gives the result in $(13 \mathrm{~b})$.

To prove sufficiency, calculate the derivatives of the demand function in (13a):

$$
p^{\prime}(x)=-\eta \alpha x^{-\eta-1}-\theta \beta x^{-\theta-1} \quad \text { and } \quad p^{\prime \prime}(x)=\eta \alpha x^{-\eta-2}+\theta(\theta+1) \beta x^{-\theta-2}
$$

Hence the elasticity and convexity can be written as:

$$
\varepsilon=\frac{f+1}{\eta f+\theta} \quad \text { and } \quad \rho=\frac{\eta(\eta+1) f+\theta(\theta+1)}{\eta f+\theta}
$$

where $f=F(x, \alpha, \beta, \eta, \theta) \equiv \frac{\alpha}{\beta} x^{\eta-\theta}$. Equation (B11) clearly satisfies condition (11a) from Proposition 2 for $\alpha$ and $\beta$, so the bipower inverse demand manifold is invariant with respect to these two parameters. Eliminating $f$ gives the explicit expression for the manifold, which completes the proof of sufficiency: $\bar{\rho}(\varepsilon)=$ $\eta+\theta+1-\eta \theta \varepsilon$.

To prove necessity, assume the manifold is affine, so $\rho(x)=a+b \varepsilon(x)$ where $a$ and $b$ are constants. Substituting for $\rho(x)$ and $\varepsilon(x)$ and collecting terms yields:

$$
x^{2} p^{\prime \prime}(x)+a x p^{\prime}(x)-b p(x)=0
$$

To solve this second-order Euler-Cauchy differential equation, we change variables as follows: $t=\log x$ and $p(x)=g(\log x)=g(t)$. Substituting for $p(x)=g(t)$, $p^{\prime}(x)=\frac{1}{x} g^{\prime}(t)$ and $p^{\prime \prime}(x)=\frac{1}{x^{2}}\left[g^{\prime \prime}(t)-g^{\prime}(t)\right]$ into (B12) gives a linear differential equation:

$$
g^{\prime \prime}(t)+(a-1) g^{\prime}(t)-b g(t)=0
$$

Assuming a trial solution $g(t)=e^{\lambda t}$ gives the characteristic polynomial: $\lambda^{2}+(a-$ 1) $\lambda-b=0$, whose roots are $\lambda=\frac{1}{2}\left[-(a-1) \pm \sqrt{(a-1)^{2}+4 b}\right]$. Only real roots make sense, so we assume $(a-1)^{2}+4 b \geq 0$. If the inequality is strict, the roots are distinct and the general solution is given by $g(t)=\alpha e^{\lambda_{1} t}+\beta e^{\lambda_{2} t}$, where $\alpha$ and $\beta$ are constants of integration. If $(a-1)^{2}=-4 b$, the roots are equal and the general solution is given by $g(t)=(\alpha+\beta t) e^{\lambda t}$. In both cases, the solution may be found by switching back from $t$ and $g(t)$ to $\log x$ and $p(x)$, recalling that $e^{\lambda \log x}=x^{\lambda}$. Hence, in the first case, $p(x)=\alpha x^{\lambda_{1}}+\beta x^{\lambda_{2}}$, and in the second case, $p(x)=(\alpha+\beta \log x) x^{\lambda}{ }^{49}$ The final step is to note that the sum of the roots is $\lambda_{1}+\lambda_{2}=1-a$ and their product is $\lambda_{1} \lambda_{2}=b$, which implies the relationship

\footnotetext{
${ }^{49}$ We do not present the case of equal roots separately in the statement of Proposition 3 in the text: the economic interpretation is more convenient if we view it as the limiting case of the general expression as $\eta$ approaches zero. See, for example, footnotes 21 and 23, which illustrate this for CARA and translog demands respectively.
} 
between the coefficients of the manifold and those of the implied demand function stated in the proposition. This completes the proof of (13a), while that of (13b) follows immediately by duality, as already noted.

\section{B\%. Proof of Proposition 4: Bipower Superconvexity}

Substituting from the bipower inverse demand manifold in (13a) into the condition for superconvexity, $\rho \geq \frac{\varepsilon+1}{\varepsilon}$, yields:

$$
\rho-\frac{\varepsilon+1}{\varepsilon}=-\frac{1}{\varepsilon}(\eta \varepsilon-1)(\theta \varepsilon-1)
$$

Next, following the approach of Proposition 2, we write the elasticity of demand in terms of a sub-function: $\varepsilon=\frac{\alpha x^{-\eta}+\beta x^{-\theta}}{\eta \alpha x^{-\eta}+\theta \beta x^{-\theta}}=\frac{f+1}{\eta f+\theta}$, where $f \equiv \frac{\alpha}{\beta} x^{\theta-\eta}$. Substituting into (B14) yields:

$$
\rho-\frac{\varepsilon+1}{\varepsilon}=\left(\frac{\eta-\theta}{\eta f+\theta}\right)^{2} \frac{f}{\varepsilon}
$$

Hence, superconvexity requires that $f$ must be positive, and so $\alpha$ and $\beta$ must have the same sign. Since at least one of them must be positive, this implies that they must both be positive for superconvexity, which proves the first part of Proposition 4.

Similarly, substituting from the bipower direct demand manifold in (13b) into the condition for superconvexity yields:

$$
\rho-\frac{\varepsilon+1}{\varepsilon}=-\frac{1}{\varepsilon^{2}}(\varepsilon-\nu)(\varepsilon-\sigma)
$$

Once again we eliminate the terms in $\varepsilon$ in parentheses using $\varepsilon=\frac{\nu \gamma p^{-\nu}+\sigma \delta p^{-\sigma}}{\gamma p^{-\nu}+\delta p^{-\sigma}}=$ $\frac{\nu g+\sigma}{g+1}$, where $g \equiv \frac{\gamma}{\delta} p^{\sigma-\nu}$. This yields:

$$
\rho-\frac{\varepsilon+1}{\varepsilon}=\left(\frac{\nu-\sigma}{g+1}\right)^{2} \frac{g}{\varepsilon^{2}}
$$

It follows that both $\gamma$ and $\delta$ must be positive for superconvexity, which proves the second part of Proposition 4.

\section{B8. Examples of Bipower Direct Demands}

\section{Properties of Pollak Demands}

With $\nu=0$ in the bipower direct case, the elasticity of demand becomes $\varepsilon=$ $\frac{\sigma \delta p^{-\sigma}}{\gamma+\delta p^{-\sigma}}=\sigma \frac{\sigma \delta p^{-\sigma}}{x}=\sigma \frac{x-\gamma}{x}$. It follows that $\sigma, \delta$ and $x-\gamma$ must have the same sign. The sign of $\sigma$ also determines whether the inverse demand function is 
logconvex or not. The CARA demand function is the limiting case when $\sigma \rightarrow 0$ : the direct demand function becomes $x=\gamma^{\prime}+\delta^{\prime} \log p, \delta^{\prime}<0$, which implies that the inverse demand function is $\log$-linear: $\log p=\alpha+\beta x, \beta<0 .{ }^{50}$ The CARA manifold is $\bar{\rho}(\varepsilon)=\frac{1}{\varepsilon}$, which is a rectangular hyperbola through the point $\{1.0,1.0\}$. Hence the CARA function is the dividing line between two sub-groups of demand functions and their corresponding manifolds, with $\sigma$ either negative or positive. For negative values of $\sigma, \gamma$ is an upper bound to consumption: the best-known example of this class is the linear demand function, corresponding to $\sigma=-1$. By contrast, for strictly positive values of $\sigma, \gamma$ is the lower bound to consumption and there is no upper bound. Especially in the LES case, it is common to interpret $\gamma$ as a "subsistence" level of consumption, but this requires that it be positive, which (when $\sigma$ and $\delta$ are positive) only holds if demand is superconvex. All members of the Pollak family with positive $\sigma$ are translatedCES functions, and, as the arrows in Figure 5(a) indicate, they asymptote towards the corresponding "untranslated-CES" function as sales rise without bound; for example, the LES demand function, with $\sigma$ equal to one, asymptotes towards the Cobb-Douglas. Table B1 summarizes the three possible cases of this family of demand functions.

Table B1-Properties of Pollak Demand Functions

\begin{tabular}{ccc}
\hline & \multicolumn{2}{c}{$\gamma<0$} \\
\hline$\sigma>0, \delta>0$ & 1. Superconvex; logconvex: $x>\gamma>0$ & 2. Subconvex; logconvex \\
$\sigma<0, \delta<0$ & 3. Subconvex; logconcave: $\gamma>x>0$ & $\mathrm{n} / \mathrm{a}$ \\
\hline
\end{tabular}

Pollak showed that these are the only demand functions that are consistent with both additive separability and quasi-homotheticity (so the expenditure function exhibits the "Gorman Polar Form"). Just as (13b) is dual to (13a), so the Pollak family of direct demand functions is dual to the Bulow-Pfleiderer family of inverse demand functions. An implication of this is that, corresponding to the property of Bulow-Pfleiderer demands that marginal revenue is linear in price, Pollak demands exhibit the property that the marginal loss in revenue from a small increase in price is linear in sales. ${ }^{51}$ This implies that the coefficient of absolute risk aversion for these demands is hyperbolic in sales, which is why, in the theory of choice under uncertainty, they are known as "HARA" ("hyperbolic

\footnotetext{
${ }^{50}$ As noted by Pollak, this demand function was first proposed by Chipman (1965), who showed that it is implied by an additive exponential utility function. Later independent developments include Bertoletti (2006) and Behrens and Murata (2007). Differentiating the Arrow-Pratt coefficient of absolute risk aversion defined in footnote 52 gives $\frac{\partial A(x)}{\partial x}=-\frac{u^{\prime} u^{\prime \prime \prime}-\left(u^{\prime \prime}\right)^{2}}{\left(u^{\prime \prime}\right)^{2}}=-\frac{p p^{\prime \prime}-\left(p^{\prime}\right)^{2}}{\left(p^{\prime}\right)^{2}}=1-\varepsilon \rho$, so absolute risk aversion is constant if and only if $\varepsilon=\frac{1}{\rho}$.

${ }^{51}$ As we show in Appendix B9 below, Bulow-Pfleiderer demands $p(x)=\alpha+\beta x^{-\theta}$ satisfy the property: $p+x p^{\prime}=\theta \alpha+(1-\theta) p$. Switching variables, we can conclude that Pollak demands $x(p)=\gamma+\delta p^{-\sigma}$ satisfy the property: $x+p x^{\prime}=\sigma \gamma+(1-\sigma) x$.
} 
absolute risk aversion") demands following Merton (1971). ${ }^{52}$

\section{Properties of PIGL Demands}

With $\nu=1$, the elasticity of demand becomes $\varepsilon=\frac{\gamma p^{-1}+\sigma \delta p^{-\sigma}}{\gamma p^{-1}+\delta p^{-\sigma}}$. Subtracting one gives: $\varepsilon-1=\frac{(\sigma-1) \delta p^{-\sigma}}{\gamma p^{-1}+\delta p^{-\sigma}}=(\sigma-1) \frac{p x-\gamma}{p x}$. It follows that $\sigma-1, \delta$ and $p x-\gamma$ must have the same sign. In addition, the demand manifold is $\rho=\frac{(\sigma+2) \varepsilon-\sigma}{\varepsilon^{2}}$, so convexity is increasing in $\sigma$. Combining these results with Proposition 4 , there are three possible cases of this demand function. For $\sigma$ less than one, the demand function is less convex than the translog (i.e., PIGLOG) case, $\delta$ is negative and $\gamma$ is positive. For $\sigma$ greater than one, $\delta$ is positive, the demand function is more convex than the translog case, and it is subconvex if $\gamma$ is negative, otherwise it is superconvex. These properties are dual to those of the inverse PIGL demand functions in Appendix B9, and, like the latter, they can be related to whether the elasticity of marginal revenue with respect to price is greater or less than one (the value of one corresponding to the PIGLOG case). Note finally that the limiting case of PIGL demand function when $\sigma$ approaches zero is the LES, the only demand function that is a subset of both PIGL and Pollak. The LES case is special in another respect: as can be seen in Figure 5(a), it is the only member of the PIGL family for which $\varepsilon$ is monotonic in $\rho$ along the manifold. In all other cases the manifold is vertical at $\{\varepsilon, \rho\}=\left\{\frac{2 \sigma}{\sigma+2}, \frac{(\sigma+2)^{2}}{4 \sigma}\right\}$. For $\sigma<0$ it is not defined for $\rho<\frac{(\sigma+2)^{2}}{4 \sigma}$, while for $\sigma>0$ it is not defined for $\rho>\frac{(\sigma+2)^{2}}{4 \sigma}$.

\section{QMOR Demand Functions}

Diewert (1976) introduced the quadratic mean of order $r$ expenditure function, which implies a general functional form for homothetic demand functions. Feenstra (2014) considers a symmetric special case and shows how it can be adapted to allow for entry and exit of goods, so making it applicable to models of monopolistic competition. In our notation, the resulting family of demand functions, taking a "firm's-eye view", is:

$$
x(p)=\gamma p^{-(1-r)}+\delta p^{-\frac{2-r}{2}}
$$

This is clearly a member of the bipower direct family, with $\nu=1-r$ and $\sigma=\frac{2-r}{2}$. Hence, from Proposition 3, its demand manifold is:

$$
\bar{\rho}(\varepsilon)=\frac{(2-r)(3 \varepsilon-1+r)}{2 \varepsilon^{2}}
$$

\footnotetext{
52 The Arrow-Pratt coefficient of absolute risk aversion is $A(x) \equiv-\frac{u^{\prime \prime}(x)}{u^{\prime}(x)}$. With additive separability this becomes $A(x)=-\frac{p^{\prime}(x)}{p(x)}=-\frac{1}{p x^{\prime}(p)}$. Using the result from footnote 51, this implies that with Pollak demands, $A(x)=\frac{1}{\sigma(x-\gamma)}$, which is hyperbolic in $x$.
} 
In the limit as $r \rightarrow 0$, this becomes $\bar{\rho}(\varepsilon)=\frac{3 \varepsilon-1}{\varepsilon^{2}}$, which is the translog manifold discussed in the text. Figure B2 illustrates this demand manifold for a range of values of $r$. For $r=2$ it coincides with the $\rho=0$ vertical line: i.e., a linear demand function from the firm's perspective. For negative values of $r$ (i.e., more convex than the translog), the manifolds extend into the superconvex region. However, this is for arbitrary values of $\gamma$ and $\delta$. Feenstra (2014) shows that these parameters, which depend on real income and on prices of other goods, must be of opposite sign when the demand function (B18) is derived from expenditure minimization. Hence, from Proposition 4, QMOR demands are not consistent with superconvexity, though in other respects they allow for considerable flexibility in modeling homothetic demands.

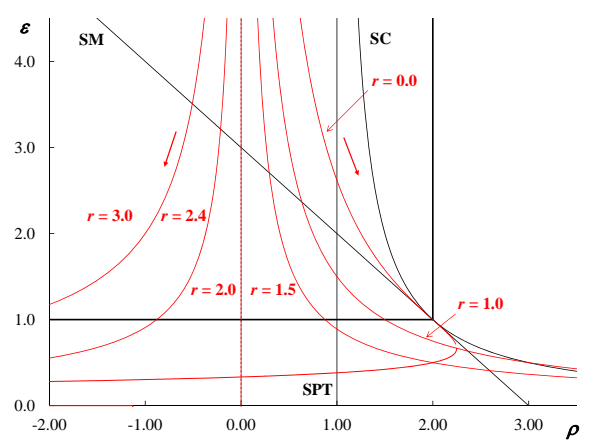

Figure B2. Demand Manifolds for QMOR Demand Functions

\section{B9. Examples of Bipower Inverse Demands}

\section{Properties of Bulow-Pfleiderer Demands}

As noted in the text, the first sub-case of the bipower inverse demand functions in (13a) we consider comes from setting $\eta$ equal to zero, giving the iso-convex or "constant pass-through" family of Bulow and Pfleiderer (1983): $p(x)=\alpha+\beta x^{-\theta}$. Convexity $\rho$ equals a constant $\theta+1$, so from $(7) \frac{1}{1-\theta}$ measures the degree of absolute pass-through for this system. Pass-through can be more than one-for one, as in the CES case $\left(\alpha=0, \theta=\frac{1}{\sigma}>0\right)$; exactly one-for-one, as in the log-linear direct demand case $\left(\theta \rightarrow 0\right.$, so $p(x)=\alpha^{\prime}+\beta^{\prime} \log x$, implying that $\log x(p)=\gamma+\delta p)$; or less than one-for-one, as in the case of linear demand $(\theta=-1$ so exactly half of a cost increase is passed through to prices).

This family has many other attractive properties. It is necessary and sufficient for marginal revenue to be affine in price. (See below.) It can be given a discrete choice interpretation: it equals the cumulative demand that would be generated 
by a population of consumers if their preferences followed a Generalized Pareto Distribution. $^{53}$ Finally, as shown by Weyl and Fabinger (2013) and empirically implemented by Atkin and Donaldson (2012), it allows the division of surplus between consumers and producers to be calculated without knowledge of quantities. Figure B3(a) shows the demand manifolds for some members of this family. ${ }^{54}$

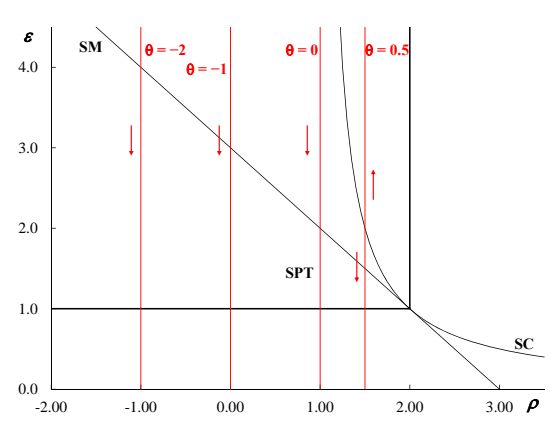

(a) The Bulow-Pfleiderer Family

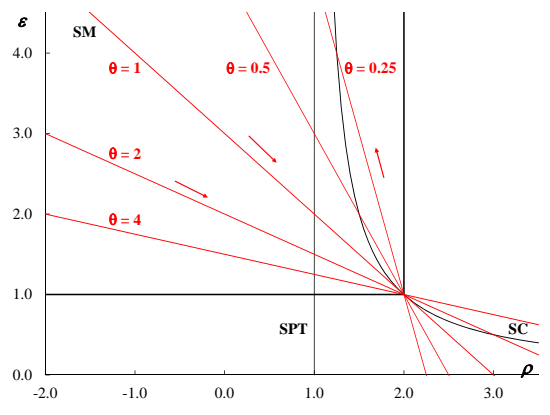

(b) The Inverse PIGL Family

Figure B3. Demand Manifolds for Some Bipower Inverse Demand Functions

With $\eta=0$, the elasticity of demand becomes: $\varepsilon=\frac{\alpha+\beta x^{-\theta}}{\theta \beta x^{-\theta}}=\frac{p}{\theta \beta x^{-\theta}}=\frac{p}{\theta(p-\alpha)}$. It follows that $\theta, \beta$ and $p-\alpha$ must have the same sign. The sign of $\theta$ also determines whether the direct demand function is logconvex (i.e., whether it exhibits superpass-through) or not: recall that $\rho-1=\theta$. There are therefore three possible cases of this demand function: see Table B2. As shown by Bulow and Pfleiderer (1983), these demands are necessary and sufficient for marginal revenue to be affine in price. Sufficiency is immediate: marginal revenue is $p+x p^{\prime}=\theta \alpha+(1-\theta) p$. Necessity follows by solving the differential equation $p(x)+x p^{\prime}(x)=a+b p(x)$, which yields $p(x)=\frac{a}{1-b}+c_{1} x^{b-1}$, where $c_{1}$ is a constant of integration.

Table B2-Properties of Bulow-Pfleiderer Demand Functions

\begin{tabular}{ccc}
\hline & $\alpha>0$ & $\alpha<0$ \\
\hline$\theta>0, \beta>0$ & 1. Superconvex; logconvex: $p>\alpha>0$ & 2. Subconvex; logconvex \\
$\theta<0, \beta<0$ & 3. Subconvex; logconcave: $\alpha>p>0$ & $\mathrm{n} / \mathrm{a}$ \\
\hline
\end{tabular}

\footnotetext{
${ }^{53}$ See Bulow and Klemperer (2012).

${ }^{54}$ Note how the behavior implied by these manifolds differs from the Pollak case in Figure 5(a), especially in the super-pass-through region. With Bulow-Pfleiderer demands, firms diverge from the CES benchmark along the SC locus as sales increase, whereas with Pollak demands they converge towards it; both these statements hold whether demands are super- or subconvex. This allows a simple visualization of the limiting behavior of a monopolistically competitive sector as market size increases without bound.
} 


\section{Properties of Inverse PIGL Demands}

The second case of (13a) considered in the text comes from setting $\eta$ equal to one, which yields the "inverse PIGL" ("price-independent generalized linear") system: $p(x)=\frac{1}{x}\left(\alpha+\beta x^{1-\theta}\right)$. This system implies that the elasticity of marginal revenue defined in footnote 15 is constant and equal to $\theta: \eta=1$ implies from (13a) that $-\frac{x R^{\prime \prime}}{R^{\prime}}=\frac{2-\rho}{\varepsilon-1}=\theta$. The limiting case as $\theta \rightarrow 1$ is the inverse "PIGLOG" ("price-independent generalized logarithmic") or inverse translog, $p(x)=\frac{1}{x}\left(\alpha^{\prime}+\right.$ $\left.\beta^{\prime} \log x\right) .{ }^{55}$ This implies that the elasticity of marginal revenue is unity, and so, as noted in Mrázová and Neary (2018), it coincides with the supermodularity locus: $\eta=\theta=1$ implies from (13a) that $\bar{\rho}(\varepsilon)=3-\varepsilon$. Figure B3(b) shows the demand manifolds for some members of this family.

With $\eta=1$, so the elasticity of demand becomes $\varepsilon=\frac{\alpha x^{-1}+\beta x^{-\theta}}{\alpha x^{-1}+\theta \beta x^{-\theta}}$, its value less one can be written in two alternative ways: $\varepsilon-1=\frac{(1-\theta) \beta x^{1-\theta}}{\alpha+\theta \beta x^{1-\theta}}=(1-\theta) \frac{p x-\alpha}{\theta p x+(1-\theta) \alpha}$. It follows that $1-\theta, \beta$ and $p x-\alpha$ must have the same sign. (Recall that $\theta$ itself equals $\frac{2-\rho}{\varepsilon-1}$ and so must be positive in the admissible region.) The value of $1-\theta$ also determines whether the demand function is supermodular or not: substituting from the demand manifold $\bar{\rho}(\varepsilon)=2+(1-\varepsilon) \theta$ into the condition for supermodularity gives $\varepsilon+\rho>3 \Leftrightarrow(\varepsilon-1)(1-\theta)>0 \Leftrightarrow \theta<1$. Combining these results with Proposition 4 shows that there are three possible cases of this demand function, as shown in Table B3.

Table B3-Properties of Inverse Pigl Demand Functions

\begin{tabular}{ccc}
\hline & $\alpha>0$ & $\alpha<0$ \\
\hline$\theta<1, \beta>0$ & 1. Superconvex; supermodular: $p x>\alpha>0$ & 2. Subconvex; supermodular \\
$\theta>1, \beta<0$ & 3. Subconvex; submodular: $\alpha>p x>0$ & $\mathrm{n} / \mathrm{a}$ \\
\hline
\end{tabular}

\section{B10. Inverse Exponential Demand}

In this section we introduce a demand function, the inverse exponential, which is an example that, for the same parameter values, is sometimes sub- and sometimes superconvex: ${ }^{56}$

$$
p(x)=\alpha+\beta \exp \left(-\gamma x^{\delta}\right)
$$

\footnotetext{
${ }^{55}$ To show this, take the limit as in footnote 23.

${ }^{56}$ Mrázová and Neary (2018) consider the properties of R\&D cost functions of this form.
} 


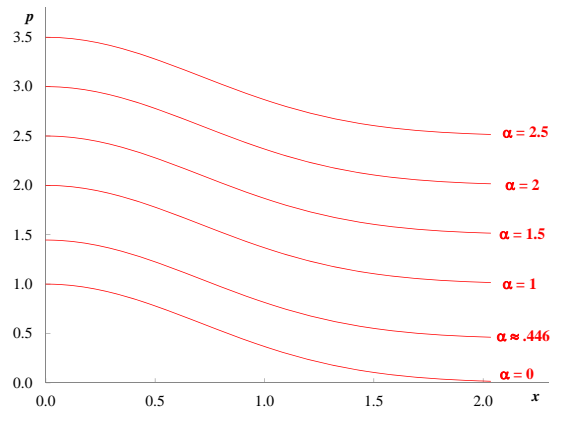

(a) Demand Functions

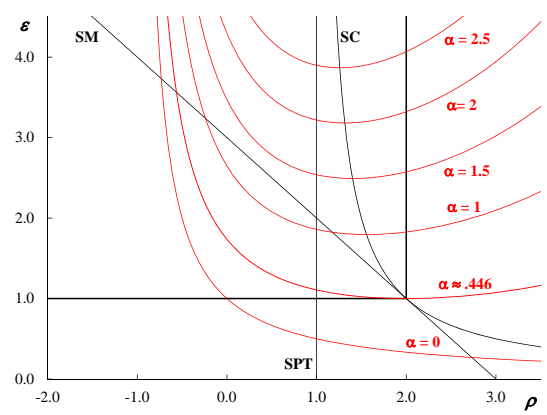

(b) Demand Manifolds

Figure B4. Inverse Exponential Demand Functions and Manifolds

where $\gamma>0$ and $\delta>0$. The elasticity and convexity of demand are found to be:

$$
\varepsilon(x)=\frac{1+\frac{\alpha}{\beta} \exp (f)}{\delta f} \quad \text { and } \quad \rho(x)=\delta f-\delta+1
$$

where $f \equiv F(x, \alpha)=\gamma x^{\delta}$. Eliminating $f$ yields a closed-form expression for the demand manifold:

$$
\bar{\varepsilon}(\rho)=\frac{1+\frac{\alpha}{\beta} \exp \left(\frac{\rho+\delta-1}{\delta}\right)}{\rho+\delta-1}
$$

This is invariant with respect to $\gamma$, in accordance with Proposition 2, and it also depends only on the ratio of $\alpha$ and $\beta$, not on their levels, in accordance with Corollary 2. Differentiating with respect to $\rho$ shows that, provided $\frac{\alpha}{\beta}$ is strictly positive, the demand function is subconvex for low values of $\rho$, which from (B21) implies low values of $x$, but superconvex for high $\rho$ and $x$ :

$$
\bar{\varepsilon}_{\rho}=\frac{-\delta+\frac{\alpha}{\beta}(\rho-1) \exp \left(\frac{\rho+\delta-1}{\delta}\right)}{\delta(\rho+\delta-1)^{2}}
$$

Figure B4 illustrates some demand functions and the corresponding manifolds from this class for a range of values of $\alpha$, assuming $\beta=1$ and $\delta=2$. A superconvex range in the admissible region is possible only for parameter values such that the minimum point of the manifold lies above the Cobb-Douglas point, $\{\varepsilon, \rho\}=\{1,2\}$, i.e., only for $\alpha>\beta \delta \exp \left(-\frac{\delta+1}{\delta}\right)$, which for the values of $\beta$ and $\delta$ underlying Figure B4 is approximately $\alpha>0.446$. For such values of $\alpha$, the manifolds are horizontal where they cross the SC locus, in accordance with Figure 1(b). 


\section{B11. Proof of Lemma 1: Uniqueness of the Translog}

We wish to show that the translog is the only demand function with a manifold of the "contiguous bipower" form $\rho=a_{1} \varepsilon^{\kappa}+a_{2} \varepsilon^{\kappa+1}$ that is always both strictly subconvex and strictly supermodular in the interior of the admissible region. The proof proceeds by showing that these conditions require that the demand manifold satisfy three distinct restrictions. These enable us to isolate the translog demand function as the only candidate.

First, it is clear by inspection that, if a demand function is always both subconvex and supermodular, then its manifold must pass through the Cobb-Douglas point, $\{\varepsilon, \rho\}=\{1,2\}$. Hence the parameters must satisfy $a_{1}+a_{2}=2$.

Second, the slope of the manifold, $\frac{d \rho}{d \epsilon}=a_{1} \kappa \varepsilon^{\kappa-1}+a_{2}(\kappa+1) \varepsilon^{\kappa}$, must be greater than that of the SM locus and less than that of the SC locus at $\{1,2\}$. Both of these slopes equal $-1:\left.\frac{d \rho}{d \epsilon}\right|_{S M}=-1$ everywhere, and $\left.\frac{d \rho}{d \epsilon}\right|_{S C}=-\frac{1}{\varepsilon^{2}}=-1$ at $\{1,2\}$. Hence the parameters must satisfy $a_{1} \kappa+a_{2}(\kappa+1)=-1$. This and the previous restriction can be solved for $a_{1}$ and $a_{2}$ in terms of $\kappa: a_{1}=3+2 \kappa$ and $a_{2}=-(1+2 \kappa)$.

Third, the curvature of the manifold, $\left.\frac{d^{2} \rho}{d \epsilon^{2}}\right|_{M}=a_{1}(\kappa-1) \kappa \varepsilon^{\kappa-2}+a_{2} \kappa(\kappa+1) \varepsilon^{\kappa-1}$, must be greater than that of the SM locus and less than that of the SC locus at $\{1,2\}$. These curvatures are: $\left.\frac{d^{2} \rho}{d \epsilon^{2}}\right|_{S M}=0$ everywhere, and $\left.\frac{d^{2} \rho}{d \epsilon^{2}}\right|_{S C}=\frac{1}{\varepsilon^{3}}=1$ at $\{1,2\}$. Hence the parameters must satisfy $0 \leq a_{1}(\kappa-1) \kappa+a_{2} \kappa(\kappa+1) \leq 1$. Substituting for $a_{1}$ and $a_{2}$ and simplifying gives: $0 \leq-2 \kappa(\kappa+2) \leq 1$. Only two integer values of $\kappa$ satisfy these inequalities: $\kappa=0$ is the SM locus itself, which is not in the interior of the admissible region; that leaves $\kappa=-2$, implying $\rho=-\varepsilon^{-2}+3 \varepsilon^{-1}=\frac{3 \varepsilon-1}{\varepsilon^{2}}$, the translog demand manifold, as was to be proved.

\section{B12. Demand Functions that are not Manifold-Invariant}

In this section we introduce two new demand systems whose demand manifolds can be written in closed form, though they depend on all the parameters, and so are not manifold invariant. We consider in turn: the "Doubly-Translated CES" super-family, which nests both the Pollak and Bulow-Pfleiderer families; and the "Translated Bipower Inverse" super-family, which nests both the "APT" (Adjustable pass-through) system of Fabinger and Weyl (2012) and a new family that we call the inverse "iso-temperance" system. ${ }^{57}$

\footnotetext{
${ }^{57} \mathrm{~A}$ third super-family is the dual of the second, the "Translated Bipower Direct" super-family. Reversing the roles of $p$ and $x$ in equation (B26) below leads to a "dual" manifold giving the inverse elasticity $e$ as a function of the direct convexity $r$ with the same form as (B28). Special cases of this include the dual of the APT system and the direct "iso-temperance" system (i.e., the demand system necessary and sufficient for $-p x^{\prime \prime \prime} / x^{\prime \prime}$ to be constant). It does not seem possible to express the manifold $\bar{\varepsilon}(\rho)$ in closed form for this family.
} 


\section{The "Doubly-Translated CES" Super-Family}

We can nest the Pollak and Bulow-Pfleiderer families as follows: $p(x)=\alpha+$ $\beta(x-\gamma)^{-\theta}{ }^{58}$ The elasticity and convexity of this function are:

$$
\varepsilon(x)=\frac{1}{\theta} \frac{p}{p-\alpha} \frac{x-\gamma}{x} \quad \rho(x)=(\theta+1) \frac{x}{x-\gamma}
$$

When $\gamma$ is zero this reduces to the Bulow-Pfleiderer case. Assuming $\gamma \neq 0$, we have $\rho \neq \theta+1$, and so the expression for $\rho$ in (B24) can be solved for $x$ : $x=\frac{\rho}{\rho-(\theta+1)} \gamma$. Substituting into the expression for $\varepsilon$ yields: ${ }^{59}$

$$
\bar{\varepsilon}(\rho)=\left[1+a_{1}\left(\frac{1}{\rho-a_{2}}\right)^{a_{3}}\right] \frac{a_{4}}{\rho}
$$

where $a_{1}=\frac{\alpha}{\beta}\{(\theta+1) \gamma\}^{\theta}, a_{2}=\theta+1, a_{3}=\theta$, and $a_{4}=\frac{\theta+1}{\theta}$. This is a closed-form expression for the manifold but it depends on all four parameters, except in special cases such as the Pollak family, when, with $\alpha=0$, it reduces to $\bar{\varepsilon}(\rho)=\frac{\theta+1}{\theta} \frac{1}{\rho}$. Nevertheless, the general demand manifold (B25) allows for considerable economy of information: three of its four parameters depend only on the exponent $\theta$ in the demand function, and the fourth parameter, $a_{1}$, is invariant to rescalings of the demand function parameters which keep $\frac{\alpha}{\beta} \gamma^{\theta}$ constant.

\section{The "Translated Bipower Inverse" Super-Family}

This demand function adds an intercept $\alpha_{0}$ to the bipower inverse family given by $(13 \mathrm{a})$ :

$$
p(x)=\alpha_{0}+\alpha x^{-\eta}+\beta x^{-\theta}
$$

Differentiating gives the elasticity and convexity:

$$
\varepsilon(x)=\frac{\alpha_{0} x^{\eta}+\alpha+\beta x^{\eta-\theta}}{\eta \alpha+\theta \beta x^{\eta-\theta}} \quad \rho(x)=\frac{\eta(\eta+1) \alpha+\theta(\theta+1) \beta x^{\eta-\theta}}{\eta \alpha+\theta \beta x^{\eta-\theta}}
$$

Assuming as before that $\rho \neq \theta+1$, and also that $\eta \neq \theta$, we can invert $\rho(x)$ to solve for $x: x(\rho)=\left[\frac{\eta \alpha}{\theta \beta} \frac{(\eta+1)-\rho}{\rho-(\theta+1)}\right]^{\frac{1}{\eta-\theta}}$. Substituting into $\varepsilon(x)$ gives a closed-form expression for the manifold:

$$
\bar{\varepsilon}(\rho)=\frac{\rho-a_{1}}{a_{2}}+\left(a_{3}-\rho\right)^{a_{4}}\left(\rho-a_{5}\right)^{a_{6}} a_{7}
$$

\footnotetext{
${ }^{58}$ After we developed this family, we realized that it had already been considered in the working paper version of Zhelobodko et al. (2012), who call it the "Augmented-HARA" system.

${ }^{59}$ Here and elsewhere, the parameters must be such that, when the exponent (here $\theta$ ) is not an integer, the expression which is raised to the power of that exponent is positive.
} 
where $a_{1}=\eta+\theta+1, a_{2}=-\eta \theta, a_{3}=\eta+1, a_{4}=\frac{\eta}{\eta-\theta}, a_{5}=\theta+1, a_{6}=$ $-\frac{\theta}{\eta-\theta}$, and $a_{7}=\left(\frac{\eta}{\beta}\right)^{\frac{\eta}{\eta-\theta}}\left(\frac{\theta}{\alpha}\right)^{-\frac{\theta}{\eta-\theta}} \frac{\alpha_{0}}{\eta \theta(\eta-\theta)}$. In general, this depends on the same five parameters as the demand function (B26), though once again it allows for considerable economy of information: all but $a_{7}$ depend only on the two exponents $\eta$ and $\theta$, and $a_{7}$ itself is unaffected by changes in the other three demand-function parameters that keep $\alpha^{\frac{\theta}{\eta-\theta}} \beta^{\frac{-\eta}{\eta-\theta}} \alpha_{0}$ constant. Equation (B28) is best understood by considering some special cases:

(1) Bipower Inverse: The cost in additional complexity of the "translation" parameter $\alpha_{0}$ is apparent. Setting this equal to zero, the expression simplifies to give the bipower inverse manifold as in Proposition 3: $\bar{\rho}(\varepsilon)=1+\eta+\theta-\eta \theta \varepsilon$.

(2) APT Demands: Fabinger and Weyl (2012) show that the pass-through rate (in our notation, $\frac{d p}{d c}=\frac{1}{2-\rho}$ ) is quadratic in the square root of price if and only if the inverse demand function has the form of (B26) with $\eta=2 \theta$. This reduces the number of parameters by one, so the demand manifold simplifies to: $\bar{\varepsilon}(\rho)=\frac{1+3 \theta-\rho}{2 \theta^{2}}-\frac{[(2 \theta+1)-\rho]^{2}}{\rho-(\theta+1)} \frac{2 \alpha}{\beta^{2} \theta^{2}} \alpha_{0}$.

(3) Iso-Temperance Demands: Setting $\eta=-1$ is sufficient to ensure that temperance, $\chi \equiv-\frac{x p^{\prime \prime \prime}}{p^{\prime \prime}}$, is constant, equal to $\theta+2$. It is also necessary. To see this, write $x p^{\prime \prime \prime}=-\chi p^{\prime \prime}$, where $\chi$ is a constant, and integrate three times, which yields $p(x)=c_{0}+c_{1} x+\frac{c_{2}}{(1-\chi)(2-\chi)} x^{2-\chi}$, where $c_{0}, c_{1}$ and $c_{2}$ are constants of integration. This is identical to (B26) with $\eta=-1$ and $\theta=\chi-2$. Note that iso-convexity implies iso-temperance, but the converse does not hold; just as CES implies iso-convexity, but the converse does not hold.

These special cases and the general demand manifold in (B28) allow us to infer the comparative statics implications of this family of demand functions. Moreover, if we are mainly interested in pass-through, we do not need to work with the demand manifold at all, since the key conditions in (7) and (B4) do not depend on the elasticity of demand (a point stressed by Weyl and Fabinger (2013)). In such cases, our approach can be applied to the slope rather than the level of demand. By relating the elasticity and convexity of this slope to each other, we can construct a "demand-slope manifold" corresponding to any given demand function, and the properties of this manifold are very informative about when pass-through is increasing or decreasing with sales. In ongoing work, we show that the demand-slope manifolds of the APT and iso-temperance demand functions are particularly convenient in this respect.

\section{B13. Calculating the Effects of Globalization}

To solve for the results in (24), use (22) to eliminate $\hat{x}$ from (20) and then solve (20) and (21) for $\hat{p}$ and $\hat{y}$, with $\hat{n}$ determined residually by (23). The results in (25) are obtained by using $\hat{x}=\hat{y}-\hat{k}$ and $\hat{N}=\hat{k}+\hat{n}$.

These results are for infinitesimal changes only. For finite changes, it is still true that the values of $\varepsilon$ and $\rho$ determine the results. However, their values are 
not fixed in general, so it is necessary to integrate the change in the dependent variable along a path taking account of the changes in $\varepsilon$ and $\rho$.

\section{B14. Change in Real Income: Details}

With symmetric preferences and identical prices for all goods, the budget constraint becomes: $I=\int_{0}^{N} p(\omega) x(\omega) \mathrm{d} \omega=N p x$. So consumption of each good is: $x=\frac{I}{N p}$. Substituting into the direct utility function yields its indirect counterpart:

$$
V(N, p, I)=F\left[N u\left(\frac{I}{N p}\right)\right]
$$

We can now define equivalent income $Y(N, p)$ as the income that preserves the initial level of utility $U_{0}$ following a shock:

$$
V\left(N, p, \frac{I}{Y}\right)=U_{0}
$$

For small changes (so equivalent and compensating variations coincide), we logarithmically differentiate, with $I$ fixed (since it equals exogenous labor income), to obtain: $\hat{N}-\xi(\hat{N}+\hat{p}+\hat{Y})=0$. Rearranging gives the change in real income in (33). Note that this is independent of the function $F$.

\section{B15. Welfare with Bulow-Pfleiderer Preferences}

The Bulow-Pfleiderer sub-utility function in (35) takes a bipower form. Hence we can immediately apply equation (13a) from Proposition 3 in Section II.D, replacing $\eta$ by $-1, \theta$ by $\theta-1, \rho$ by $\frac{1}{\varepsilon}$ and $\varepsilon$ by $-\frac{1}{\xi}$ :

$$
u(x)=\alpha x+\frac{1}{1-\theta} \beta x^{1-\theta} \Leftrightarrow \frac{1}{\varepsilon}=\theta+(\theta-1)\left(-\frac{1}{\xi}\right)
$$

Rearranging gives the first equation in (36), and using the demand manifold to eliminate $\theta$ gives the second.

\section{B16. Welfare with Pollak Preferences}

\section{From Demands to Preferences}

Recall from Section II.D that the Pollak demand function is $x(p)=\gamma+\delta p^{-\sigma}$, where $\delta, \sigma$ and $x-\gamma$ have the same sign, and $\gamma$ has the same sign as $\delta$ and $\sigma$ if and only if demand is superconvex. To derive the sub-utility function we must first invert to obtain the inverse demand function. This yields: $p(x)=\left(\frac{x-\gamma}{\delta}\right)^{-\frac{1}{\sigma}}$. It is convenient to redefine the constants as $\zeta \equiv-\gamma \sigma$ and $\beta \equiv(\delta / \sigma)^{1 / \sigma}$ (i.e., we 
replace $\gamma$ by $-\zeta / \sigma$, and $\delta$ by $\left.\beta^{\delta} / \sigma\right)$, which yields: $p(x)=\beta(\sigma x+\zeta)^{-\frac{1}{\sigma}}$. Both $\beta$ and $\sigma x+\zeta$ are positive. Integrating and setting the constant of integration equal to zero yields the sub-utility function (37). We have already seen in Proposition 4 that all bipower direct demand functions are superconvex if and only if both $\gamma$ and $\delta$ are positive, and in Section B 8 that with Pollak demands $\sigma$ and $\delta$ must have the same sign. Hence a negative value of $\zeta \equiv-\gamma \sigma$ is necessary and sufficient for demand to be superconvex.

\section{Gains and Losses from Globalization with Pollak Preferences}

Substituting for $\xi=\frac{\varepsilon \rho-2}{\varepsilon}$ into the general expression for welfare change in equation (34) gives in this case:

$$
\hat{Y}=\frac{\varepsilon}{\varepsilon \rho-2}\left[1-\frac{(\varepsilon-1)^{2}}{\varepsilon^{2}(2-\rho)}\right] \hat{k}
$$

This is negative when $\rho>\rho^{Y} \equiv \frac{\varepsilon^{2}+2 \varepsilon-1}{\varepsilon^{2}}$. To confirm that this lies in the admissible range $\rho \in[\underline{\rho}, \bar{\rho}] \equiv\left[\frac{2}{\varepsilon}, 1+\frac{2}{\varepsilon}\right]$, note that $\rho^{Y}-\underline{\rho}=\frac{\varepsilon^{2}-1}{\varepsilon^{2}}>0$ and $\bar{\rho}-\rho^{Y}=\frac{1}{\varepsilon^{2}}>0$.

\section{Alternative Normalizations of the Sub-Utility Function}

In the text we follow Pollak (1971) and Dixit and Stiglitz (1977) and set the constant of integration in the sub-utility function equal to zero. As Dixit and Stiglitz (1979) point out, this need not imply that $u(0)$ is strictly positive: we can define $u(x)=\max \left\{0, \frac{\beta}{\sigma-1}(\sigma x+\zeta)^{\frac{\sigma-1}{\sigma}}\right\}$, which is discontinuous at $x=0$, but in all respects is a valid utility index. However, different authors take different views on whether it is satisfactory that new goods provide a finite level of utility, even when they are consumed in infinitesimal (though strictly positive) amounts. An alternative approach, due to Pettengill (1979), is to choose the constant of integration itself to ensure that $u(0)=0$. This implies that the sub-utility function takes the following form:

$$
u(x)=\frac{\beta}{\sigma-1}\left[(\sigma x+\zeta)^{\frac{\sigma-1}{\sigma}}-\zeta^{\frac{\sigma-1}{\sigma}}\right]
$$

Note, however, that $\zeta$ must be positive, which implies as already noted that demand is always subconvex: a zero level of consumption is not in the consumer's feasible set if $\zeta$ is negative. Hence this normalization of the Pollak utility function implies a different restriction on the feasible region from that in the text, with the whole of the superconvex region now inadmissible.

The elasticity and convexity of demand are unaffected by this re-normalization of the sub-utility function. However, the elasticity of utility is very different. ${ }^{60}$ It

\footnotetext{
${ }^{60}$ Utility is ordinal, so preferences and demands are invariant to monotonic transformations of the
} 


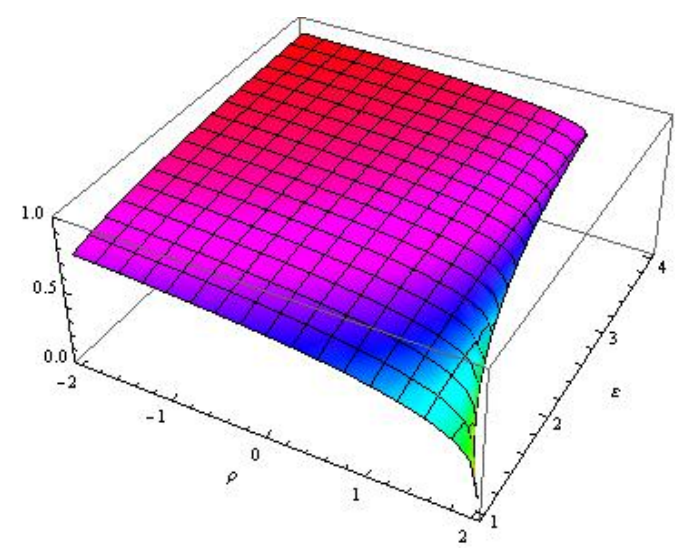

(a) Elasticity of Utility

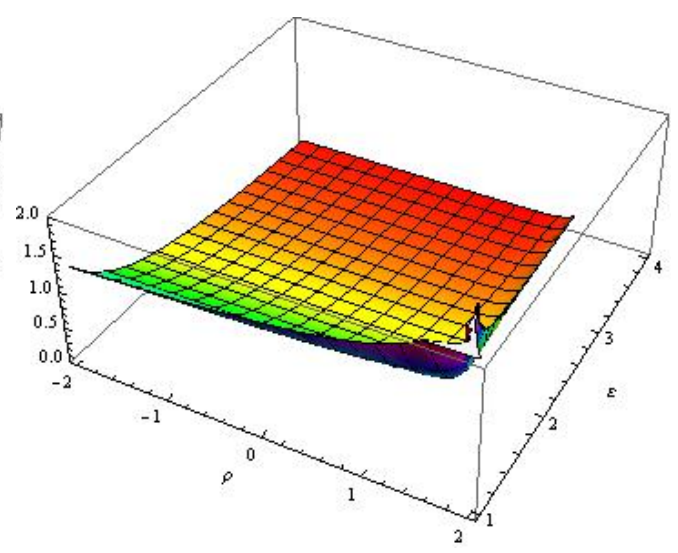

(b) Change in Real Income

Figure B5. Globalization and Welfare: Normalized Pollak Preferences

now behaves more like the Bulow-Pfleiderer case, except that it is not consistent with superconvex demands:

$$
\xi^{N}=H \xi, \quad H(\varepsilon, \sigma)=\frac{1}{1-\left(\frac{\varepsilon-\sigma}{\varepsilon}\right)^{\frac{\sigma-1}{\sigma}}}, \quad H(\varepsilon, \rho)=\frac{1}{1-\left(\frac{\varepsilon-\varepsilon \rho+1}{\varepsilon}\right)^{\frac{\varepsilon \rho-2}{\varepsilon \rho-1}}}
$$

where $H$ is a correction factor applied to the unnormalized elasticity of utility given in equation (38). The results are shown in Figure B5. Compared with Figure 9 in the text, the main differences are that the elasticity of utility now lies between zero and one for all admissible values of $\varepsilon$ and $\rho$, i.e., throughout the subconvex region, and that the gains from globalization are always positive. Both the elasticity of utility and the change in real income behave qualitatively with respect to $\varepsilon$ and $\rho$ in a similar fashion to the case of Bulow-Pfleiderer preferences in Figure 8. All this confirms that the elasticity and convexity of demand are not sufficient statistics for the welfare effects of globalization, and that small changes in the parameterization of utility can have major implications for the quantitative effects of changes in the size of the world economy.

\section{B17. Markup and Pass-Through Data}

Table B4 summarizes the data on the markups $m$ and pass-through elasticities $k$ from De Loecker et al. (2016) that we use and gives the implied values of $\varepsilon$ and $\rho$. The mean and median estimates of the markup $m$ are taken from their

overall utility function, i.e., to different choices of the $F$ function, in equation (15). However, utility and its derivatives, and hence the measured gains from trade, are not invariant to monotonic transformations of the sub-utility function, as here. 
Table VI (p. 483). They measure markups as $\frac{p}{c}$, which we here adjust to $m \equiv \frac{p-c}{c}$. The estimated pass-through coefficients $k$ are taken from their Table VII (p. 488). From Column (1) of that table we take the OLS estimate of 0.337 with a standard error of 0.041 , implying a $95 \%$ confidence interval of 0.257 to 0.417 . From Column (2), which instruments marginal costs with input tariffs and lagged marginal costs, we take the IV estimate of 0.305 with a standard error of 0.084 , implying a $95 \%$ confidence interval of 0.140 to 0.470 . Column (3) instruments marginal costs with input tariffs and two-period lagged marginal costs: it yields a point estimate that lies within the OLS confidence interval but is much less precisely estimated, with a $95 \%$ confidence interval that implies values for $\rho$ extending outside the admissible region.

The OLS estimates are biased when marginal costs and prices are jointly determined. However, they may nevertheless be of interest for two reasons. First, while the results from de Loecker et al. (2016) showing the effects of output tariffs on markups use a second-order polynomial to control for marginal costs, they note that the results are very similar if marginal costs are assumed to be constant. (See their page 494, footnote 53.) Second, while the OLS estimate of the pass-through elasticity may be biased in principle, it is not very different from the IV estimate in practice: recall that the point estimates are 0.337 and 0.305 respectively, and each is comfortably within the confidence interval of the other.

TABle B 4 - VAlues of $\varepsilon$ AND $\rho$ IMPlied By DAta ON $m$ AND $k$

\begin{tabular}{cccccc}
\hline & \multicolumn{2}{c}{ OLS Estimate of $k$} & & \multicolumn{2}{c}{ IV Estimate of $k$} \\
\cline { 2 - 3 } \cline { 5 - 6 } & Mean $m$ & Median $m$ & & Mean $m$ & Median $m$ \\
\hline$m$ & 1.70 & 0.34 & & 1.70 & 0.34 \\
$k^{*}$ & 0.337 & 0.337 & & 0.305 & 0.305 \\
& $(0.257,0.417)$ & $(0.257,0.417)$ & & $(0.140,0.470)$ & $(0.140,0.470)$ \\
\hline \multirow{2}{*}{$\varepsilon$} & 1.588 & 3.941 & & 1.588 & 3.941 \\
& 0.901 & -0.214 & & 0.786 & -0.447 \\
$\rho^{*}$ & $(0.557,1.113)$ & $(-0.908,0.212)$ & & $(-0.639,1.211)$ & $(-3.317,0.411)$ \\
\hline
\end{tabular}

Note: Sources: $m$ and $k$ : De Loecker et al. (2016); $\varepsilon$ and $\rho$ : authors' calculations (see text). * Point estimates, with implied $95 \%$ confidence intervals in parentheses.

\section{B18. Pigou's Law}

Following Deaton (1974), assume an additively separable utility function with a finite number of goods:

$$
U(x)=F\left[\Sigma_{i} u\left(x_{i}\right)\right]
$$


Maximizing this subject to the budget constraint yields the first-order conditions:

$$
u^{\prime}\left(x_{i}\right)=\lambda p_{i}
$$

where $\lambda$ is the marginal utility of income adjusted for the units of measurement of utility in (B35) by deflating by $F^{\prime}$. Totally differentiating this and expressing the results in terms of proportional changes gives:

$$
\varepsilon_{u^{\prime} x_{i}} \hat{x}_{i}=\hat{\lambda}+\hat{p}_{i}
$$

where $\varepsilon_{u^{\prime} x_{i}} \equiv \frac{d \log u^{\prime}\left(x_{i}\right)}{d \log x_{i}}$ is the elasticity of the slope of the sub-utility function with respect to the consumption of good $x_{i}$.

If the exogenous shock to the consumer is a change in income (so $\hat{p}_{i}=0$ ), equation (B37) implies:

$$
\varepsilon_{u^{\prime} x_{i}} \eta_{i}=\Phi^{-1}
$$

where $\eta_{i}$ is the income elasticity of demand for $\operatorname{good} i$ and $\Phi \equiv\left[\frac{d \log \lambda}{d \log I}\right]^{-1}$ is the inverse of the elasticity of the marginal utility of income with respect to income, or Frisch's "flexibility of the marginal utility of money". If instead the shock is a change in the price of good $j$, equation (B37) implies:

$$
\varepsilon_{u^{\prime} x_{i}} \varepsilon_{i j}=\varepsilon_{\lambda j}+\delta_{i j}
$$

where $\varepsilon_{i j}$ is the cross-price elasticity of demand for good $i$ with respect to the price of good $j$, and $\delta_{i j}$ is the Kronecker delta. Multiplying (B39) by the budget share of good $i, \omega_{i}$, and summing over all goods $j$ yields:

$$
-\varepsilon_{u^{\prime} x_{i}} \omega_{j}=\varepsilon_{\lambda j}+\omega_{j} \eta_{j}
$$

where we use the aggregation conditions $\Sigma_{i} \omega_{i}=1$ and $\Sigma_{i} \omega_{i} \varepsilon_{i j}=-\omega_{j}$. Finally, substituting into (B38) and eliminating $\varepsilon_{u^{\prime} x_{i}}$ yields:

$$
\varepsilon_{i j}=\left[\delta_{i j}-\omega_{j}\left(\Phi^{-1}+\eta_{j}\right)\right] \Phi \eta_{i}
$$

When $i=j$, this gives the desired relationship between the own-price and income elasticities. It is approximately proportional, with the deviation from proportionality depending on the budget share of good $i$. In the continuum case, the budget share of any good is infinitesimal and so (B41) with $i=j$ reduces to exact proportionality as in (41) in the text. Note also that, in the continuum case, $\varepsilon_{u^{\prime} x_{i}}$ is the inverse of the demand elasticity $\varepsilon_{i i}$ : when the budget share is infinitesimal, the Frisch marginal-utility-compensated elasticity of demand, like the Hicksian utility-compensated elasticity, is identical to the Marshallian elasticity of demand. 


\section{B19. Glossary of Terms}

In this appendix we note some alternative definitions of terms that we use in the text. The text can be read independently of the glossary.

Log-Convexity: We describe a function $f(x)$ as log-convex at a point $\left(x_{0}, f\left(x_{0}\right)\right)$ if and only if $\log f(x)$ is convex in $x$ at $\left(x_{0}, f\left(x_{0}\right)\right)$. This appears to be standard practice, though there are notable exceptions: see "Superconvexity" below.

Manifold: Each of the demand manifolds we present is a one-dimensional smooth manifold, or a smooth plane curve in the Euclidean plane $R^{2}$. Each is defined by an equation $f(\varepsilon, \rho)=0$, where $f: R^{2} \rightarrow R$ is a smooth function, and the partial derivatives $\frac{\partial f}{\partial \varepsilon}$ and $\frac{\partial f}{\partial \rho}$ are never both zero. Strictly speaking, a manifold cannot have a self-intersection point, whereas the relationship between $\varepsilon$ and $\rho$ could exhibit such a feature.

Marshall's Second Law: In Book III, Chapter IV of his Principles, entitled "The Law of Elasticity," Marshall argued that the elasticity of demand increases with price. This is equivalent to what we call subconvexity, and is sometimes called "Marshall's Second Law of Demand." His First Law is, of course, that demand curves slope downwards. (A nice irony is that violations of both laws are of economic interest.) Note that this is different from Marshall's second law of derived demand: the demand for an input is likely to be less elastic the smaller its share in the cost of the output which uses it.

Pollak or HARA Demands: The demand functions due to Pollak (1971) that we consider in Section II.D are sometimes called "HARA" ("hyperbolic absolute risk aversion") demands following Merton (1971). In the present context the former label seems more appropriate. Pollak characterized the preferences that are consistent with these demands in a non-stochastic multi-good setting, showing that they are the only ones that are consistent with both additive separability and quasi-homotheticity. By contrast, Merton focused on portfolio allocation in a stochastic one-good setting.

Superconvexity: Following Mrázová and Neary (2018), we describe a function $f(x)$ as superconvex at a point $\left(x_{0}, f\left(x_{0}\right)\right)$ if and only if $\log f(x)$ is convex in $\log x$ at $\left(x_{0}, f\left(x_{0}\right)\right)$. Arkolakis et al. (2012) use the term "log-convex" for such a function, whereas Kingman (1961) uses the term "superconvex" as a synonym for the more widely-used sense of log-convexity, i.e., $\log f(x)$ convex in $x$.

\section{REFERENCES}

Arkolakis, Costas, Arnaud Costinot, David Donaldson, and Andrés Rodríguez-Clare. 2012. "The Elusive Pro-Competitive Effects of Trade." working paper, MIT, revised July 2014.

Atkin, David, and Dave Donaldson. 2012. "Who's Getting Globalized? The Size and Nature of Intranational Trade Costs." working paper, Yale University. 
Behrens, K., and Y. Murata. 2007. "General Equilibrium Models of Monopolistic Competition: A New Approach." Journal of Economic Theory, 136(1): 776-787.

Bergstrom, Ted, and Mark Bagnoli. 2005. "Log-Concave Probability and its Applications." Economic Theory, 26: 445-469.

Bertoletti, P. 2006. "Logarithmic Quasi-Homothetic Preferences." Economics Letters, 90(3): 433-439.

Bulow, Jeremy, and Paul Klemperer. 2012. "Regulated Prices, Rent Seeking, and Consumer Surplus." Journal of Political Economy, 120(1): 160-186.

Bulow, Jeremy I., and Paul Pfleiderer. 1983. "A Note on the Effect of Cost Changes on Prices." Journal of Political Economy, 91(1): 182-185.

Chipman, John S. 1965. "A Survey of the Theory of International Trade: Part 2, The Neo-Classical Theory." Econometrica, 33(4): 685-760.

Cowan, Simon. 2012. "Third-Degree Price Discrimination and Consumer Surplus." Journal of Industrial Economics, 60(2): 333-345.

Deaton, Angus. 1974. "A Reconsideration of the Empirical Implications of Additive Preferences." Economic Journal, 84(334): 338-348.

De Loecker, Jan, Pinelopi K Goldberg, Amit K Khandelwal, and Nina Pavcnik. 2016. "Prices, Markups and Trade Reform." Econometrica, 84(2): 445-510.

Diewert, W. Erwin. 1976. "Exact and Superlative Index Numbers." Journal of Econometrics, 4(2): 115-145.

Dixit, Avinash K., and Joseph E. Stiglitz. 1977. "Monopolistic Competition and Optimum Product Diversity." American Economic Review, 67(3): 297-308.

Dixit, Avinash K., and Joseph E. Stiglitz. 1979. "Monopolistic Competition and Optimum Product Diversity: Reply [to Pettengill]." American Economic Review, 69(5): 961-963.

Fabinger, Michal, and E. Glen Weyl. 2012. "Pass-Through and Demand Forms." working paper, University of Chicago.

Feenstra, Robert C. 2014. "Restoring the Product Variety and ProCompetitive Gains from Trade with Heterogeneous Firms and Bounded Productivity." NBER Working Paper No. 16796.

Kingman, J. F. C. 1961. "A Convexity Property of Positive Matrices." Quarterly Journal of Mathematics, 12(1): 283-284. 
Merton, Robert C. 1971. "Optimum Consumption and Portfolio Rules in a Continuous-Time Model." Journal of Economic Theory, 3(4): 373-413.

Mrázová, Monika, and J. Peter Neary. 2018. "Selection Effects with Heterogeneous Firms." forthcoming in Journal of the European Economic Association.

Pettengill, John S. 1979. "Monopolistic Competition and Optimum Product Diversity: Comment." American Economic Review, 69(5): 957-960.

Pollak, Robert A. 1971. "Additive Utility Functions and Linear Engel Curves." Review of Economic Studies, 38(4): 401-414.

Weyl, E. Glen, and Michal Fabinger. 2013. "Pass-Through as an Economic Tool: Principles of Incidence under Imperfect Competition." Journal of Political Economy, 121(3): 528-583.

Zhelobodko, Evgeny, Sergey Kokovin, Mathieu Parenti, and JacquesFrançois Thisse. 2012. "Monopolistic Competition: Beyond the Constant Elasticity of Substitution." Econometrica, 80(6): 2765-2784. 